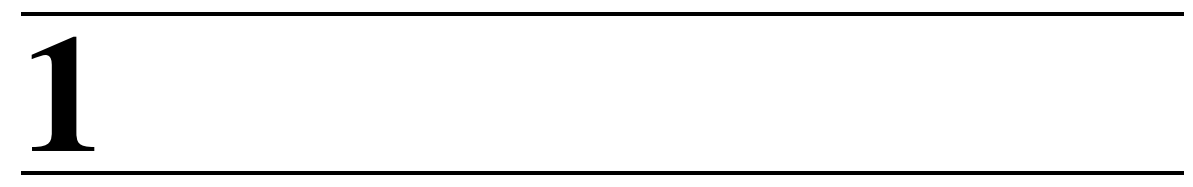

\title{
BASIC THEORY OF DISTRIBUTED POINT SOURCE METHOD (DPSM) AND ITS APPLICATION TO SOME SIMPLE PROBLEMS
}

\author{
D. Placko \\ Ecole Normale Superieure, Cachan, France \\ T. KUNDU \\ University of Arizona, USA
}

\subsection{INTRODUCTION AND HISTORICAL DEVELOPMENT OF DPSM}

In this chapter, the historical evolution of distributed point source method (DPSM) and its basic principles are presented. First, the magnetic field generated by a magnetic transducer/sensor in a free space is obtained. A U-shaped magnetic sensor with high-permeability core is first modeled (Placko and Kundu, 2001). Then, the method is extended to problem geometries with one interface (Placko et al., 2001, 2002). The source of the field is denoted as "transducer" or "sensor," and the interface between two media is sometimes called "target." Observation points that are not necessarily on the interface are also called "target points." Figure 1.1 shows the relative orientations of the transducer and interface. The interface or target can be an infinite plane or it can have a finite dimension, acting as a finite scatterer. Only one reflection by the target surface is first considered. The method is illustrated through some examples from electromagnetic and ultrasonic applications. In this chapter, different DPSM source

DPSM for Modeling Engineering Problems, Edited by Dominique Placko and Tribikram Kundu Copyright (C) 2007 John Wiley \& Sons, Inc. 


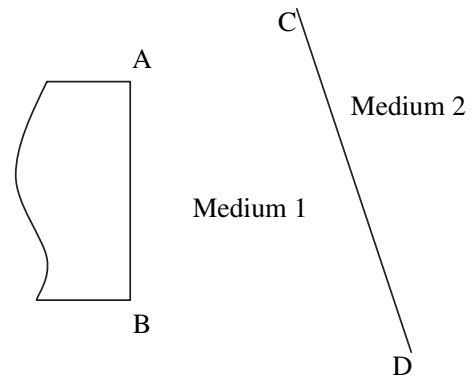

$\mathrm{AB}=$ Source or transducer or sensor $\mathrm{CD}=$ Interface or target

Figure 1.1 Problem geometry with interface.

configurations are considered including controlled-space radiation (CSR) sources and triplets, as it can be seen in the patent (Placko et al., 2002).

DPSM modeling is based on a spatial distribution of point sources and can be applied to both two-dimensional (2D) and three-dimensional (3D) problem geometries. Mostly, 3D modeling is presented in this book for magnetic, acoustic, electrostatic, and electromagnetic field problems. In the DPSM modeling technique the transducer surface and interface are replaced by a distribution of point sources, as shown in Figure 1.2a. One layer of sources is introduced near the transducer and a second layer near the interface. Point sources that model the transducer are called the "active" sources and those near the interface are called the "passive" sources. It should be noted here that a transducer generates a field and an interface alters that field by introducing reflected, transmitted, and scattered fields. If the interface is removed, the active point sources should still be present. However, if the active sources are turned off, then the passive point sources must be turned off as well because in the absence of active sources, the passive sources do not exist. Active and passive point sources can be distributed very close to the transducer face and interface, respectively, as shown in Figure 1.2a or away from them as shown in Figure 1.2b. It is also not

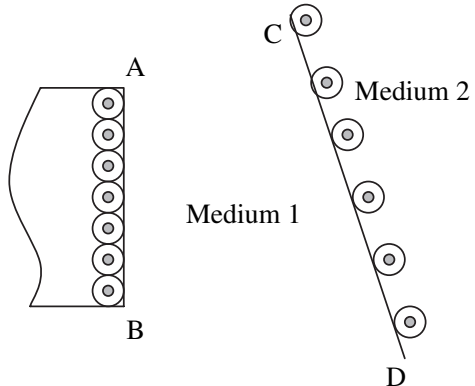

(a)

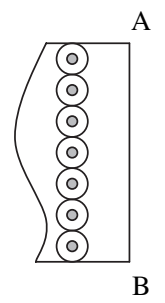

(b)

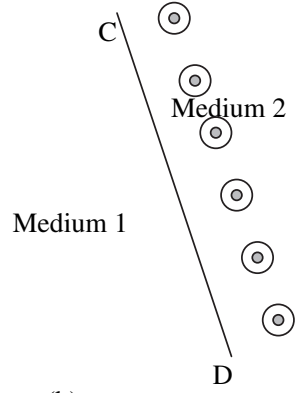

)

Figure 1.2 Synthesizing the field by placing point sources: (a) close to the sensor and interface, (b) away from the sensor and interface. 
necessary for the layer of point sources to be parallel to the surface (transducer or interface) that is modeled by these sources. Strengths of the point sources are adjusted such that the boundary conditions on the transducer surface and continuity conditions across the interface are satisfied. This can be achieved by inverting some matrices. By adjusting the point source strengths, the total field can be correctly modeled by different layers of point sources placed in different orientations. Naturally, for different orientations of the point sources, individual source strength vectors should be different. The total field is computed by adding fields generated by all active and passive point sources. Note that unlike the boundary element or finite element techniques, in this formulation the discretization of the problem boundary or of the problem domain is not necessary.

Like other numerical modeling schemes, accuracy of the computation depends on the number of point sources considered. This process of introducing a number of point sources can be called "mesh generation." In this chapter, we study the effect of the spacing between two neighboring point sources on the accuracy of the field computation and the optimum spacing for accurate numerical computation. It is shown here that for accurately modeling acoustic fields, the spacing between two neighboring point sources should be less than the acoustic wavelength (in fact, as we will see later, this condition has to be fulfilled for all kinds of waves, but the proof is given for the acoustic wave modeling). This restriction can be relaxed if we are interested in computing the field far away from the point source locations. For example, if one is interested in computing the field generated by a circular sensor of finite dimension in a homogeneous medium, the point source spacing must be a fraction of the wavelength if one is interested in computing the field accurately adjacent to the transducer face. However, at a larger distance the field can be computed accurately by considering fewer point sources of higher strength although it will not give good results near the transducer. Flat transducers or sensors with circular and rectangular cross-sections as well as point-focused concave transducers are modeled accurately by taking appropriate source spacing and are presented in this chapter.

Figures 1.3-1.5 show the steps of DPSM evolution, improvements in elemental source modeling, and different problems that have been solved so far by this technique (Placko, 1984, 1990; Placko and Kundu, 2001, 2004; Placko et al., 1985, 1989, 2002; Ahmad et al., 2003, 2005; Dufour and Placko, 1996; Lee et al., 2002; Lemistre and Placko, 2004; Banerjee et al., 2006).

\subsection{BASIC PRINCIPLES OF DPSM MODELING}

\subsubsection{The fundamental idea}

In this subsection, we first describe the basic principle of this method, which is based on the idea of using multiple point sources distributed over the active part of a sensor or an interface. Active sources synthesize the transducer-generated signals in 


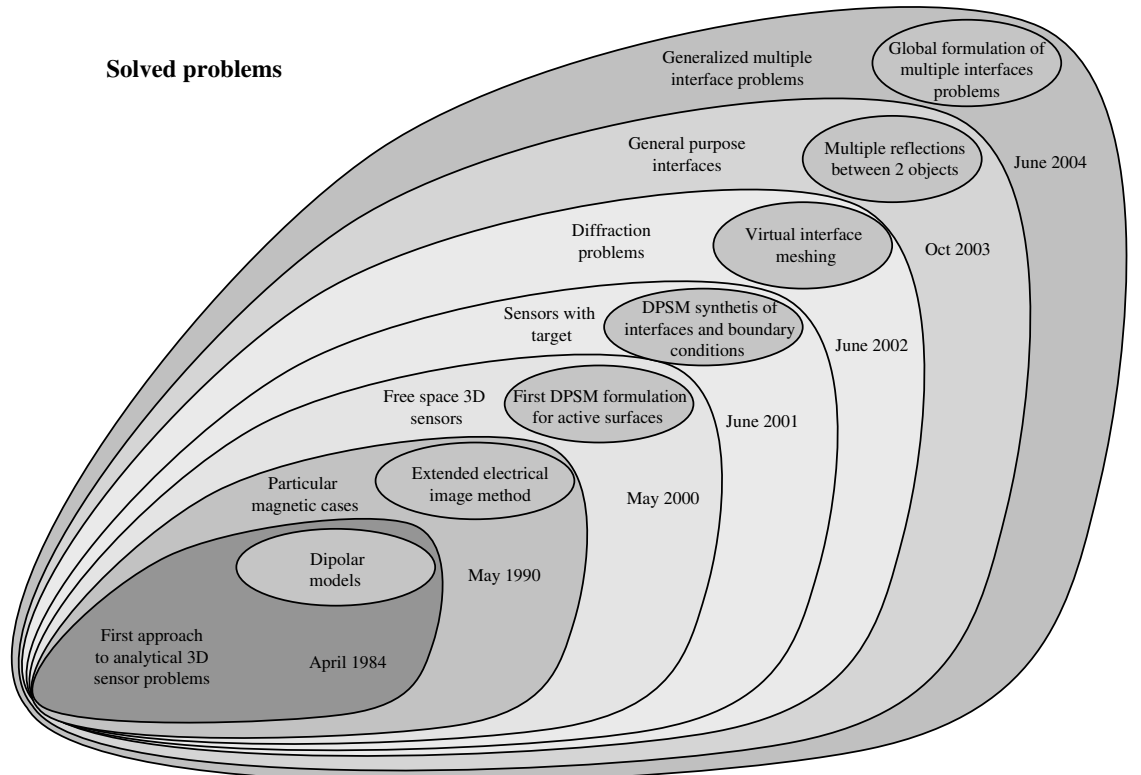

DPSM formulation improvement

Figure 1.3 DPSM birth and evolution.

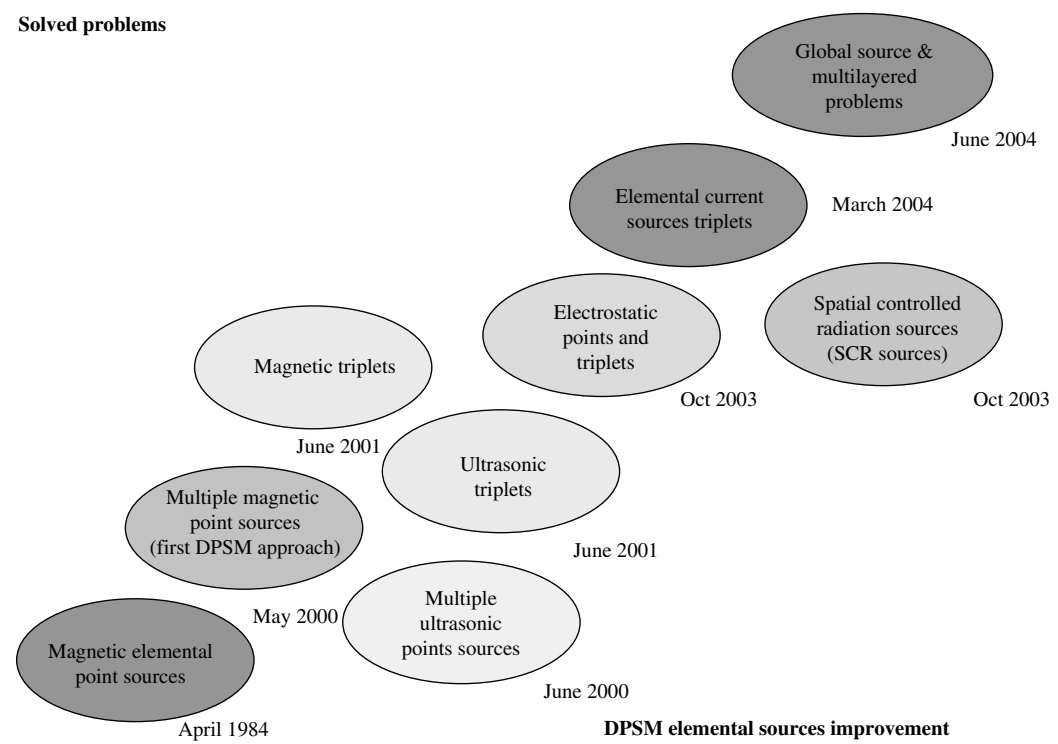

Figure 1.4 DPSM source improvement. 


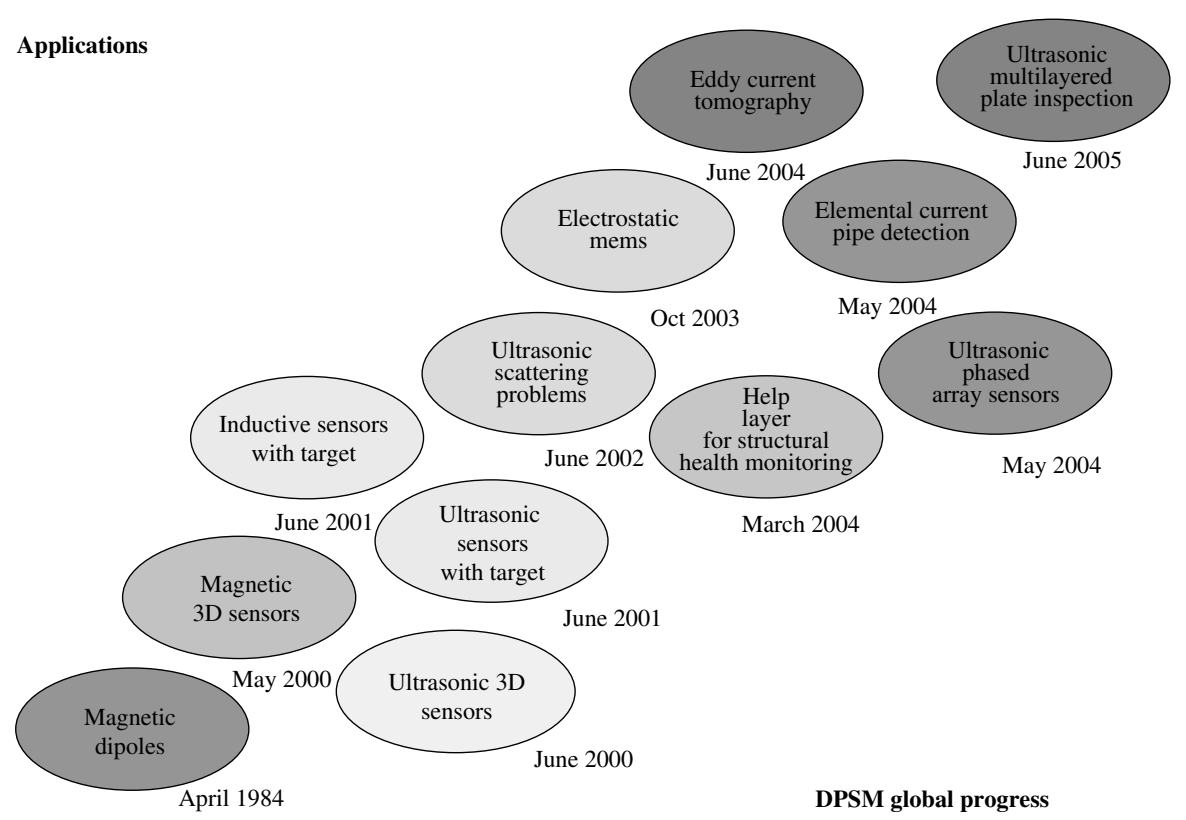

Figure 1.5 Different problems solved by DPSM.

a homogeneous medium, whereas the passive point sources distributed along the interface generate signals to model the reflection and transmission fields. For a finite interface the passive sources also model the scattered field. Because the distributed point sources model the total field, we call this method the "distributed point source method" or DPSM. It should be mentioned here that this technique is based on the analytical solutions of basic point source problems. Therefore, it can be considered as a semi-analytical technique for solving sensor problems that include magnetic, ultrasonic, and electrostatic sensors. For example, it is possible to compute the magnetic field emitted by the open magnetic core of an eddy current sensor, or acoustic pressure in front of an ultrasonic transducer without discretizing the space by a large number of 3D finite elements. Magnetic and ultrasonic sensor examples are presented in this chapter to illustrate the method because these problems have some interesting properties as discussed later. It should be noted here that for a magnetic sensor, the magnetic potential remains constant on the sensor surface and the magnetic flux varies from point to point, whereas for the acoustic sensor in a fluid, the particle velocity remains constant on the sensor surface and the acoustic pressure varies. It requires an additional matrix inversion in the magnetic field modeling, which is not necessary for the acoustic field modeling.

An elemental point source is shown in Figure 1.6. In a nonconductive medium, it involves both scalar potential and vector field, the field being proportional to the gradient of the potential. Each source is surrounded by a surface ("bubble") on which 


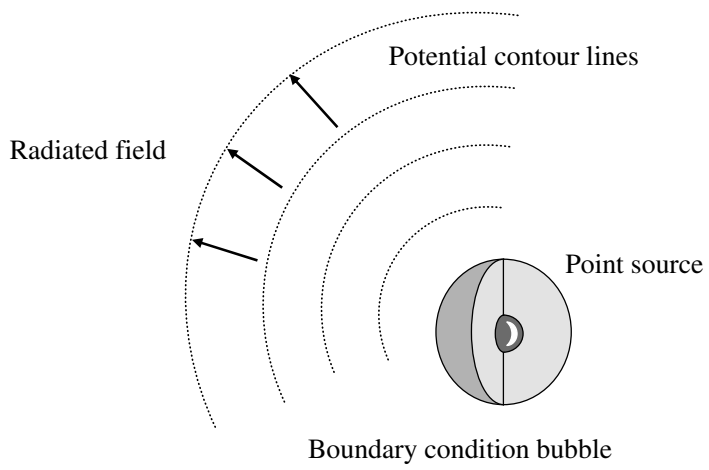

Figure 1.6 Elemental point source.

the boundary conditions are applied. Because the boundary conditions are specified on the sensor surface for active point sources and on the interface surface for the passive point sources, the bubble surface should touch those surfaces such that the transducer surface or interface are tangents to the surface. Therefore, the point sources at the centers of the bubbles cannot be located on the transducer surface or on the interface. Reason for this restriction will be discussed later.

1.2.1.1 Basic equations The basic principle of the DPSM is illustrated in Figure 1.7. The implementation of the model simply requires the replacement of the active surface of the transducer by an array of point sources, so that the initial

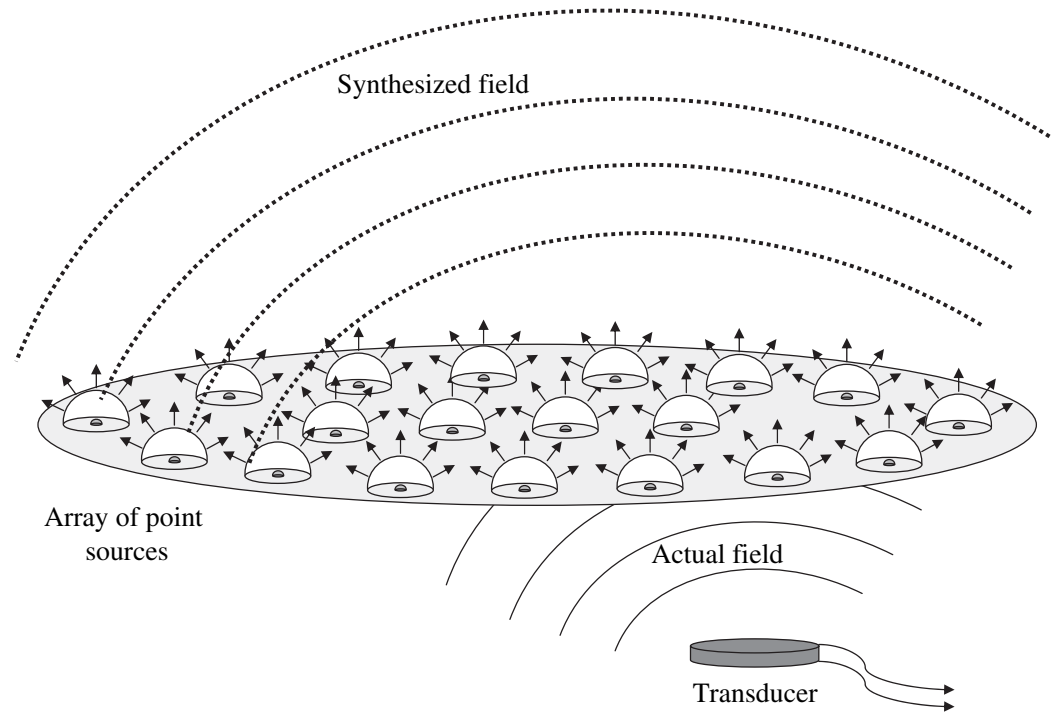

Figure 1.7 Equivalent source radiation. 


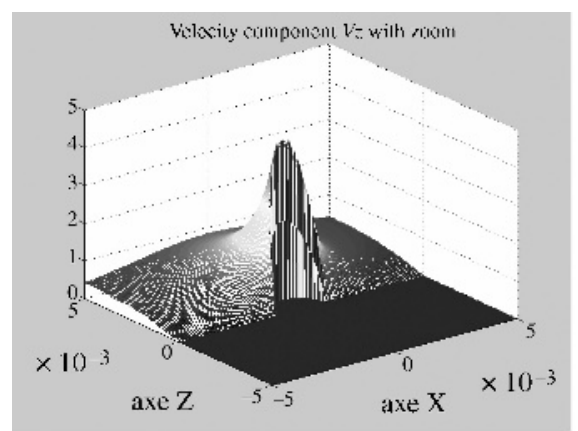

(a)

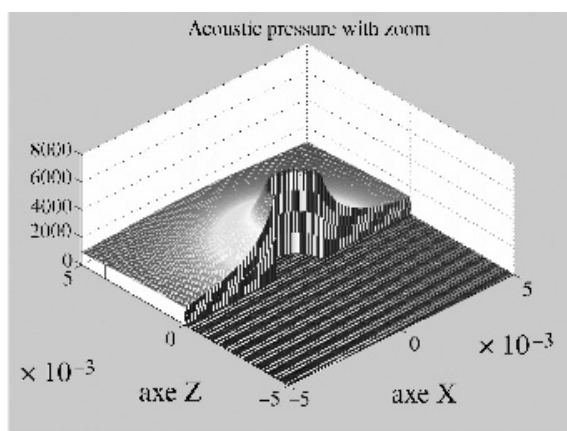

(b)

Figure 1.8 Illustration of the controlled-space radiation source properties.

complexity associated with a complex finite shape of the transducer is changed into a superposition of elementary point source problems. One way of replacing the surface by an array of point sources is discussed below.

The active surface of the transducer is discretized into a finite number of elementary surfaces $d S$, a point source is placed at the centroid of every elemental surface. The source strength and the radiation area of the sources are controlled. Unlike ordinary point sources, the sources used in DPSM do not necessarily radiate energy in all directions. For this reason these sources can be called CSR sources. For example, a source can be defined to radiate only in the bottom or top half space, or right or left half space (see Fig. 1.8).

In the generic derivation, symbols $\theta$ and $\varphi$ are used to represent different parameters for different engineering problems as described below. For magnetic sensors, $\theta$ and $\varphi$ represent the scalar magnetic potential $\Theta$ and the flux $\Phi / \mu_{0}$ of the magnetic induction $(H)$, respectively. For ultrasonic transducers, $\theta$ and $\varphi$ represent the acoustic pressure $P$ and the flux $\Phi$ of the particle displacement $(x)$, respectively. Note that the particle velocity $v=\frac{d x}{d t}$. For electrostatic systems, $\theta$ and $\varphi$ represent the scalar magnetic potential $V$ and the flux $Q / \varepsilon_{0}$ of the electric field $(E)$. The interaction function that relates the field generated by the unit source (such as the elemental charge for electrostatic problems) to $\theta$ is denoted by $f$. Table 1.1 shows the fundamental equations in different fields of engineering. It should be mentioned here that it is possible to obtain similar equivalent equations for problems from other fields of engineering such as thermal problems, for example. Nevertheless, it will be shown later that for electromagnetic waves the situation is slightly different because the sources in this case are elemental vectors of current, and in addition, the potential is often a vector and not a scalar, due to eddy currents generated in conductive media.

It is interesting to note that the energy (or the power) radiated by such a system is the product of a scalar quantity and the flux of a vector (or the time derivative of the flux, for power). Let us denote the scalar quantity by $\theta_{k}$ and the flux emitted by the point source $k$ by $\varphi_{k}$. Figure 1.9 shows how the total field at a given point is computed by adding fields generated by all the point sources. It also shows that because of 
TABLE 1.1 Some physical values in DPSM modeling

\begin{tabular}{|c|c|c|c|c|c|}
\hline & & & Surface power & $\begin{array}{l}\text { Surface } \\
\text { energy }\end{array}$ & Function $f$ \\
\hline Electrostatic & $\vec{E}=-\overrightarrow{\operatorname{grad}}(V)$ & $\iint \vec{E} \cdot \overrightarrow{d S}=\frac{Q}{\varepsilon_{0}}$ & $\frac{d Q_{S}}{d t} \times V=\frac{d D}{d t} \times V$ & $\frac{1}{2} Q_{s} V$ & $\frac{e^{-i\left(k_{\mathrm{f}} \cdot r\right)}}{2 \pi \varepsilon_{0} \cdot r}$ \\
\hline Magnetostatic & $\vec{H}=-\overrightarrow{\operatorname{grad}}(\Theta)$ & $\iint \vec{H} \cdot \overrightarrow{d S}=\frac{\Phi}{\mu_{0}}$ & $\frac{d \Phi_{s}}{d t} \times \Theta=\frac{d B}{d t} \times \Theta$ & $\frac{1}{2} \Phi_{s} \Theta$ & $\frac{e^{-i\left(k_{\mathrm{f}} \cdot r\right)}}{2 \pi \mu_{0} \cdot r}$ \\
\hline Ultrasonic & $\begin{aligned} \vec{F}_{v} & =k \cdot \vec{v}=\rho \frac{d \vec{v}}{d t} \\
& =-\overrightarrow{\operatorname{grad}}(P)\end{aligned}$ & $\iint \vec{x} \cdot \overrightarrow{d S}=\Phi$ & $\frac{d x}{d t} \times P$ & $\frac{1}{2} x P$ & $\frac{-i \omega \rho v_{0}}{2 \pi r} e^{i \cdot k_{\mathrm{f}} \cdot r}$ \\
\hline
\end{tabular}

[ $r$ is the distance of the point source, $K_{f}$ is the wave number; for electrostatic systems, $\theta$ is the scalar magnetic potential $V, \varphi$ is the flux $Q / \varepsilon_{0}$ of the electric field $(E)$; for magnetic sensors, $\theta$ is the scalar magnetic potential $\Theta, \varphi$ is the $\Phi / \mu_{0}$ of the the magnetic induction $(H)$; for ultrasonic problems, $\rho$ is the fluid density, $P$ is the pressure, $x$ is the particle displacement, $\Phi$ is the flux of particle displacement, $v_{0}$ is the transducer velocity, $\omega$ is the signal frequency.]

rotating symmetry, the elemental surface $d S$ can be changed into a hemispherical surface $d S$ with radius $r_{\mathrm{S}}\left(d S=2 \pi r_{\mathrm{S}}^{2}\right)$.

1.2.1.2 Boundary conditions One needs to introduce the boundary conditions before solving the problem. For computing the values of the flux $\varphi_{k}$ for $N$ sources, one needs $N$ number of equations. These equations are obtained by introducing

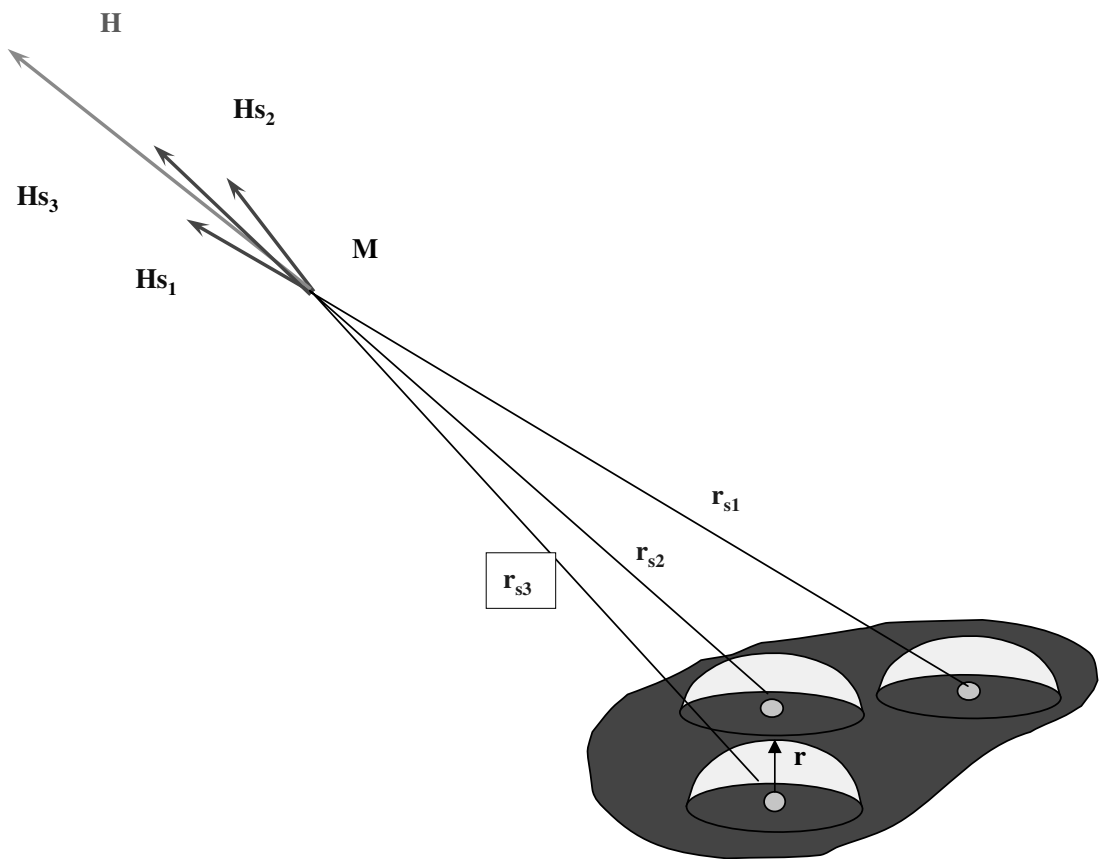

Figure 1.9 Equivalent surface discretization. 
boundary conditions on the scalar quantity $\theta_{k}$ at $N$ given points. One possible choice is to place these specific points (denoted by $P_{k}$ ) at the apex of the hemispherical surfaces (then $r_{\mathrm{S}}$ is normal to the surface). Clearly, greater is the number of points, smaller is the value of radius $r_{\mathrm{S}}$. Therefore, when $N$ tends to infinity, $r_{\mathrm{S}}$ tends to 0 , and $P_{k}$ points tend to reach the surface on which the point sources are placed. Let us now illustrate this technique to model the magnetic field generated by a magnetic sensor.

\subsubsection{Example in the case of a magnetic open core sensor}

Under assumptions of very high permeability core, the implementation of the model simply requires discretization of the active surface (magnetic poles) of the core to obtain an array of point sources. Let us denote the scalar quantity by $\theta_{k}$ and the flux emitted by the source $k$ by $\varphi_{k}$. In the application described in this section, $\theta_{k}$ and $\varphi_{k}$ represent the magnetic scalar potential and the flux of magnetic induction, respectively.

1.2.2.1 Governing equations and solution Magnetic fields emitted by open magnetic cores (electrical motors, magnetic and eddy current sensors) are modeled in this section. Solving such problems without any approximation will be very difficult in this domain of electromagnetic modeling. A magnetic sensor with a 'U'-shaped open magnetic core (see Fig. 1.10) is considered as an illustrative example.

Let us assume that the active part of a sensor is composed of two "poles"-north and south poles of the magnetic transducer, see Figure 1.11.

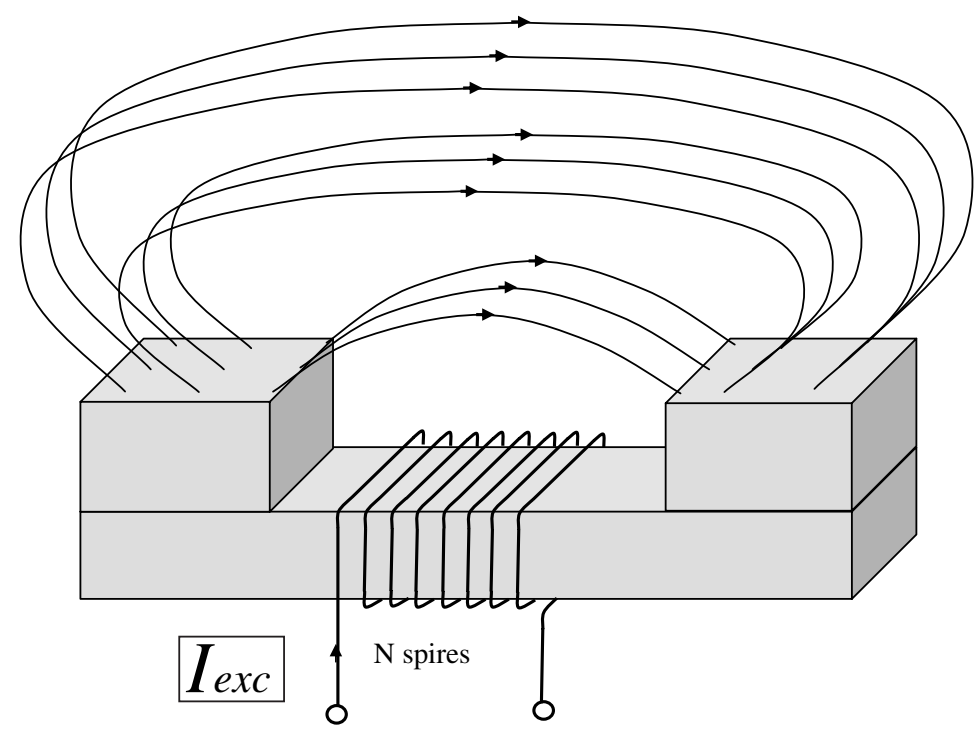

Figure 1.10 Geometry of the magnetic sensor studied. 


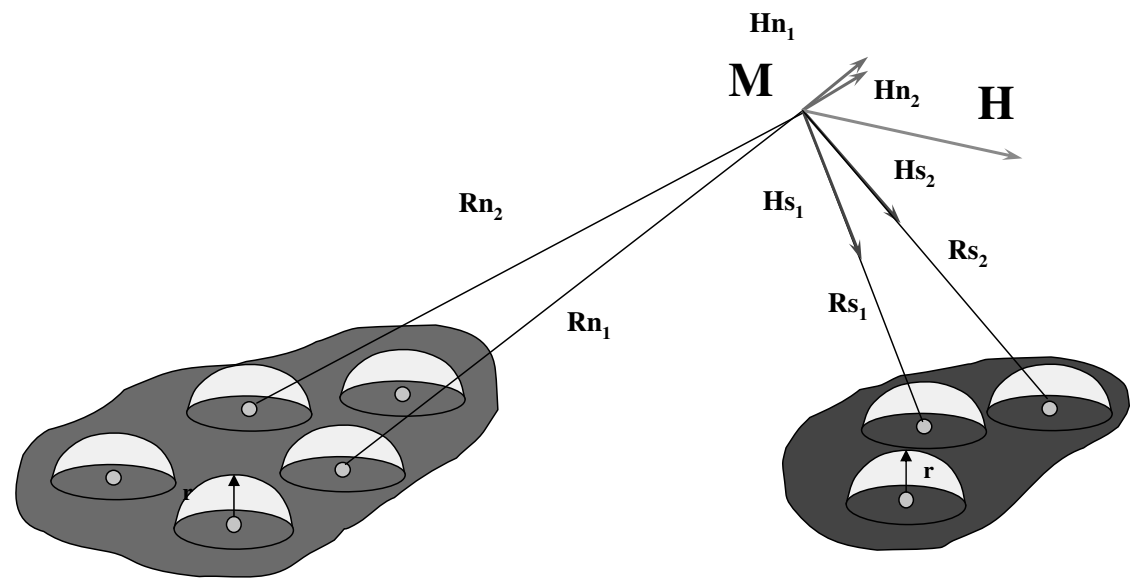

North pole

South pole

Figure 1.11 Discretization of magnetic sensor poles.

The active surfaces are discretized into elementary surfaces $d S$. At the center of the $i$ th elemental surface $d S$, a point source emitting a flux $\varphi_{i}$ is placed. From the conservation of the magnetic flux, one can write that the summation of the flux emitted by all point sources is equal to zero.

$$
\varphi_{\mathrm{N}}+\varphi_{\mathrm{S}}=\sum_{\text {pole } \mathrm{N}} \varphi_{\mathrm{N} i}+\sum_{\text {pole } \mathrm{S}} \varphi_{\mathrm{S} i}=0
$$

The magnetic potential at a given point $\mathrm{M}$ in the space is obtained by considering the contribution of all magnetic point sources.

$$
\theta=\sum_{\text {pole } \mathrm{N}} \theta_{i}+\sum_{\text {pole S }} \theta_{j}
$$

General relations between the magnetic field and the scalar magnetic potential are given by

$$
\vec{H}=\frac{-d \theta}{d \vec{r}} \quad \text { and } \quad \varphi=\iint \mu_{0} \vec{H} \cdot \overrightarrow{d S}
$$

It yields in our case, for the $i$ th point source

$$
\varphi_{i}=H_{i} \cdot 2 \pi \mu_{0} r^{2} \quad \text { and } \quad \frac{\theta^{i}=\varphi^{i}}{2 \pi \mu_{0} r}
$$


Hence, Eq. (1.2) becomes

$$
\theta=\sum_{\text {pôle } \mathrm{N}} \varphi_{\mathrm{N} i} * f\left(r_{\mathrm{N} i}\right)+\sum_{\text {pôle } \mathrm{S}} \varphi_{\mathrm{S} j} * f\left(r_{\mathrm{S} j}\right)
$$

where

$$
f(r)=\frac{1}{2 \pi \mu_{0} r}
$$

After introducing $C_{i}$ as the coordinate of the center of the source $S_{i}$, Eq. (1.5) takes the following form:

$$
\theta=\sum_{\text {pôle } \mathrm{N}} \varphi_{\mathrm{N} i} * f\left(M-C_{i}\right)+\sum_{\text {pôle S }} \varphi_{\mathrm{S} j} * f\left(M-C_{j}\right)
$$

1.2.2.2 Solution of coupling equations At this step, the value of the magnetic flux emitted by the point sources is unknown. An additional boundary condition is then introduced to obtain a new equation set. This is done by computing the magnetic potential $\theta_{k}$ at each peak point $\left(P_{k}\right)$ of the hemispherical surface (radius $r$ ), due to all sources $S_{i}$ (see Fig. 1.12).

$\theta_{k}$ can be obtained from Eq. (1.6)

$$
\theta_{k}=\sum_{\text {pôle } \mathrm{N}} \varphi_{\mathrm{N} i} * f\left(P_{k}-C_{i}\right)+\sum_{\text {pôle S }} \varphi_{\mathrm{S} j} * f\left(P_{k}-C_{j}\right)
$$

As the magnetic circuit of the sensor is composed of a material of high permeability, there is no difference in magnetic potential between the points of the same pole. Therefore, $\theta_{k}=\theta_{\mathrm{N}}=\alpha$ for the north pole and $\theta_{k}=\theta_{\mathrm{S}}=-\beta$ for the south pole, where

$$
\theta_{\mathrm{N}}-\theta_{\mathrm{S}}=\alpha+\beta=N * I_{\mathrm{exc}}
$$

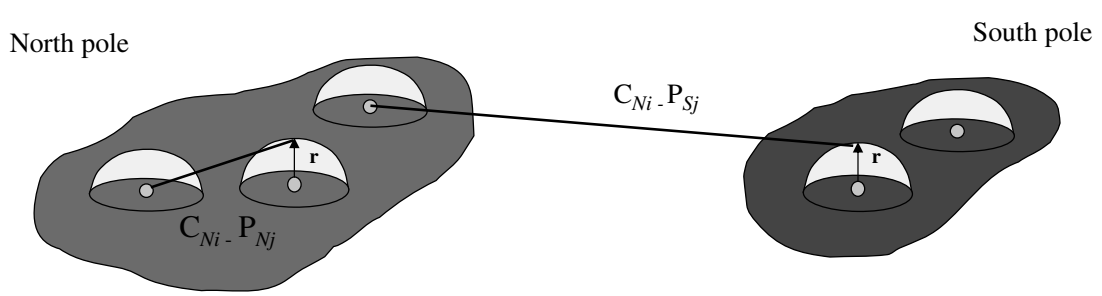

Figure 1.12 Definition of distances for coupling matrix computation. 
The number of equations thus obtained is equal to the number of point sources. Thus, one gets a square matrix for the coupling equations

$$
\begin{aligned}
\left(\begin{array}{c}
\theta_{\mathrm{Np} 1} \\
\theta_{\mathrm{Np} 2} \\
\theta_{\mathrm{Np} 3} \\
\theta_{\mathrm{Sp} 1} \\
\theta_{\mathrm{Sp} 2}
\end{array}\right)=\left(\begin{array}{c}
+\alpha \\
+\alpha \\
+\alpha \\
-\beta \\
-\beta
\end{array}\right) \\
=\left[\begin{array}{lll}
\left.\left.\left[\begin{array}{lll}
F_{\mathrm{Np} 1 \mathrm{Nc} 1} & F_{\mathrm{Np} 1 \mathrm{Nc} 2} & F_{\mathrm{Np} 1 \mathrm{Nc} 3} \\
F_{\mathrm{Np} 2 \mathrm{Nc} 1} & F_{\mathrm{Np} 2 \mathrm{Nc} 2} & F_{\mathrm{Np} 2 \mathrm{Nc} 3} \\
F_{\mathrm{Np} 3 \mathrm{Nc} 1} & F_{\mathrm{Np} 3 \mathrm{Nc} 2} & F_{\mathrm{Np} 3 \mathrm{Nc} 3}
\end{array}\right] \quad\left[\begin{array}{ll}
F_{\mathrm{Np} 1 \mathrm{Sc} 1} & F_{\mathrm{Np} 1 \mathrm{Sc} 2} \\
F_{\mathrm{Np} 2 \mathrm{Sc} 1} & F_{\mathrm{Np} 2 \mathrm{Sc} 2} \\
F_{\mathrm{Np} 3 \mathrm{Sc} 1} & F_{\mathrm{Np} 3 \mathrm{Sc} 2}
\end{array}\right]\right] *\left(\begin{array}{lll}
F_{\mathrm{Sp} 1 \mathrm{Sc} 1} & F_{\mathrm{Sp} 1 \mathrm{Sc} 2} \\
F_{\mathrm{Sp} 1 \mathrm{Nc} 1} & F_{\mathrm{Sp} 1 \mathrm{Nc} 2} & F_{\mathrm{Sp} 1 \mathrm{Nc} 3} \\
F_{\mathrm{Sp} 2 \mathrm{Nc} 1} & F_{\mathrm{Sp} 2 \mathrm{Nc} 3} & F_{\mathrm{Sp} 2 \mathrm{Nc} 3}
\end{array}\right]\right)
\end{array}\right]\left(\begin{array}{l}
\varphi_{\mathrm{N} 1} \\
\varphi_{\mathrm{N} 2} \\
\varphi_{\mathrm{N} 3} \\
\varphi_{\mathrm{S} 1} \\
\varphi_{\mathrm{S} 2}
\end{array}\right)
\end{aligned}
$$

This system of equations with four submatrices can be rewritten as:

$$
\begin{gathered}
\Theta_{\mathrm{N}}=F_{\mathrm{NN}} * \Psi_{\mathrm{N}}+F_{\mathrm{NS}} * \Psi_{\mathrm{S}} \\
\Theta_{\mathrm{S}}=F_{\mathrm{SN}} * \Psi_{\mathrm{N}}+F_{\mathrm{SS}} * \Psi_{\mathrm{S}}
\end{gathered}
$$

So that

$$
\Theta=F * \Psi
$$

Inversion of Eq. (1.10) gives the magnetic flux for all point sources

$$
\Psi=F^{-1} \Theta=G \Theta
$$

which gives

$$
\Psi=\left(\begin{array}{l}
\varphi_{\mathrm{N} 1} \\
\varphi_{\mathrm{N} 2} \\
\varphi_{\mathrm{N} 3} \\
\varphi_{\mathrm{S} 1} \\
\varphi_{\mathrm{S} 2}
\end{array}\right)=\left[\begin{array}{lll}
{\left[\begin{array}{lll}
G_{\mathrm{Np} 1 \mathrm{Nc} 1} & G_{\mathrm{Np} 1 \mathrm{Nc} 2} & G_{\mathrm{Np} 1 \mathrm{Nc} 3} \\
G_{\mathrm{Np} 2 \mathrm{Nc} 1} & G_{\mathrm{Np} 2 \mathrm{Nc} 2} & G_{\mathrm{Np} 2 \mathrm{Nc} 3} \\
G_{\mathrm{Np} 3 \mathrm{Nc} 1} & G_{\mathrm{Np} 3 \mathrm{Nc} 2} & G_{\mathrm{Np} 3 \mathrm{Nc} 3}
\end{array}\right]} & {\left[\begin{array}{ll}
H_{\mathrm{Np} 1 \mathrm{Sc} 1} & H_{\mathrm{Np} 1 \mathrm{Sc} 2} \\
H_{\mathrm{Np} 2 \mathrm{Sc} 1} & H_{\mathrm{Np} 2 \mathrm{Sc} 2} \\
H_{\mathrm{Np} 3 \mathrm{Sc} 1} & H_{\mathrm{Np} 3 \mathrm{Sc} 2}
\end{array}\right]} \\
{\left[\begin{array}{lll}
G_{\mathrm{Sp} 1 \mathrm{Nc} 1} & G_{\mathrm{Sp} 1 \mathrm{Nc} 2} & G_{\mathrm{Sp} 1 \mathrm{Nc} 3} \\
G_{\mathrm{Sp} 2 \mathrm{Nc} 1} & G_{\mathrm{Sp} 2 \mathrm{Nc} 3} & G_{\mathrm{Sp} 2 \mathrm{Nc} 3}
\end{array}\right]} & {\left[\begin{array}{ll}
H_{\mathrm{Sp} 1 \mathrm{Sc} 1} & H_{\mathrm{Sp} 1 \mathrm{Sc} 2} \\
H_{\mathrm{Sp} 2 \mathrm{Sc} 1} & H_{\mathrm{Sp} 2 \mathrm{Sc} 2}
\end{array}\right]}
\end{array}\right] *\left(\begin{array}{c}
+\alpha \\
+\alpha \\
+\alpha \\
-\beta \\
-\beta
\end{array}\right)
$$

The condition on the flux (Eq. 1.1) in combination with Eq. (1.11) gives a new equation to determine the values of $\alpha$ and $\beta$ :

$$
\varphi_{\mathrm{N}}+\varphi_{\mathrm{S}}=\alpha \sum_{i, j} G_{i j}-\beta \sum_{k, l} H_{k l}=0
$$




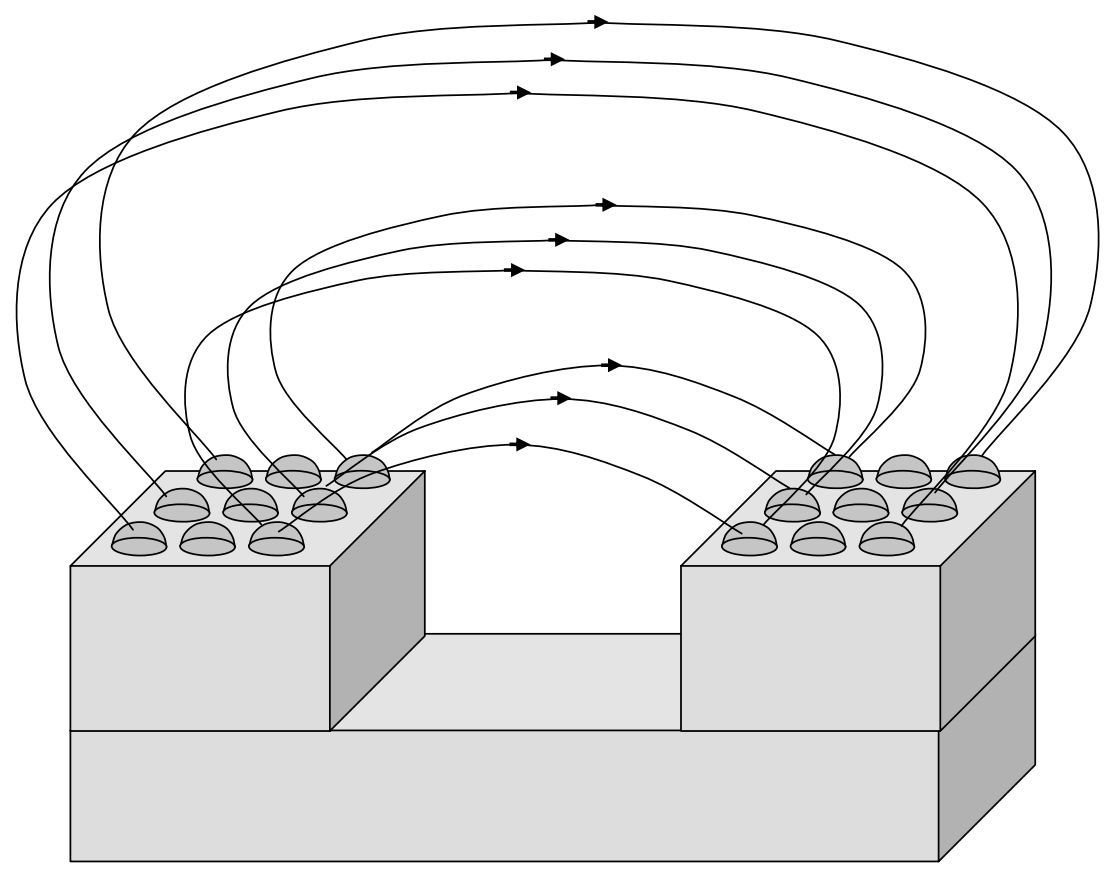

Figure 1.13 Illustration of the DPSM method with 9 sources on each pole.

After knowing the magnetic flux values, the magnetic potential or the field in the space in front of the magnetic circuit is computed from Eqs. (1.3) and (1.5). We can also compute some macroscopic parameters like the reluctance $\Re$ by

$$
\Re=\left(\theta_{\mathrm{N}}-\theta_{\mathrm{S}}\right) * \varphi_{\mathrm{N}}^{-1}=N * I_{\mathrm{exc}} * \varphi_{\mathrm{N}}^{-1}
$$

1.2.2.3 Results and discussion Some results obtained with the DPSM model are presented and compared with the results obtained by the finite element method (Ansys 3D software) (ANSYS, 1999). Geometry of the sensor, with 9 point sources for each pole, is shown in Fig. 1.13.

For this simulation, Figure 1.14a and b shows the magnetic potential at the surface of the north pole for 9 sources and 144 sources, respectively. Similar to Figure 1.13, in Figure 1.14a, a small number of sources (9 sources) is kept to illustrate the principle of the DPSM method. The boundary condition that the magnetic potential is constant at the apex of every hemispherical surface can be clearly seen in this figure. Same parameters are shown in Figure 1.14b when the number of point sources in each pole is increased to $12 \times 12=144$. Dimensions along the $x$ - and $y$-axes are given in millimeters. Figure $1.15 \mathrm{a}$ and $\mathrm{b}$ presents the normal component of the magnetic field at the surface of the north pole for 9 and 144 sources, respectively. 


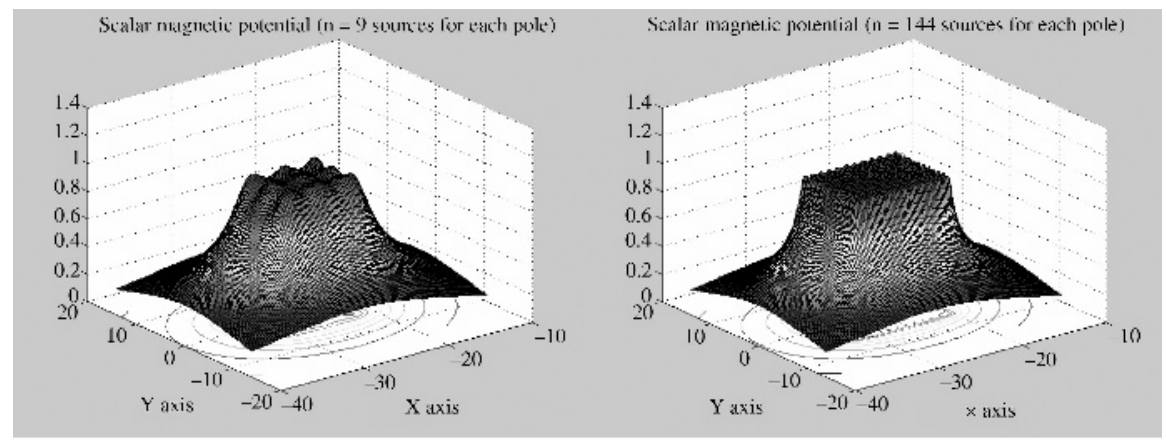

(a)

(b)

Figure 1.14 Magnetic potential on north pole for (a) 9 and (b) 144 sources on each pole.

Figure 1.16a presents the $H_{x}$ component of the magnetic field at the level of the poles, obtained with the DPSM model. Figure 1.16b shows the variation of the magnitude of $H_{x}$ along a line in the $y$-direction on the poles' plane. Figure $1.16 \mathrm{~b}$ shows the magnitude of $H_{x}$ obtained by DPSM (dashed line) and ANSYS 3D simulation (continuous line). This figure shows that the DPSM results are in good agreement with the ANSYS simulation results.

Furthermore, it should be pointed out that the DPSM synthesis of the magnetic field has been made, in this simple example, by matching the boundary conditions on the scalar term (magnetic potential) at the surface of the transducer. It should be noticed here that the satisfaction of the boundary condition gives rise to some interesting conditions on the vector term (magnetic field) because the scalar and vector terms are linked by a gradient relation (see Eq. (1.3)). Therefore, a first-order development of the scalar term applied to the neighboring points of the boundary surface guarantees a matching of the first-order derivative terms in any tangential direction to the boundary surface. In addition, it should be noted that the surface does not correspond to the wave front; therefore, every point, where the

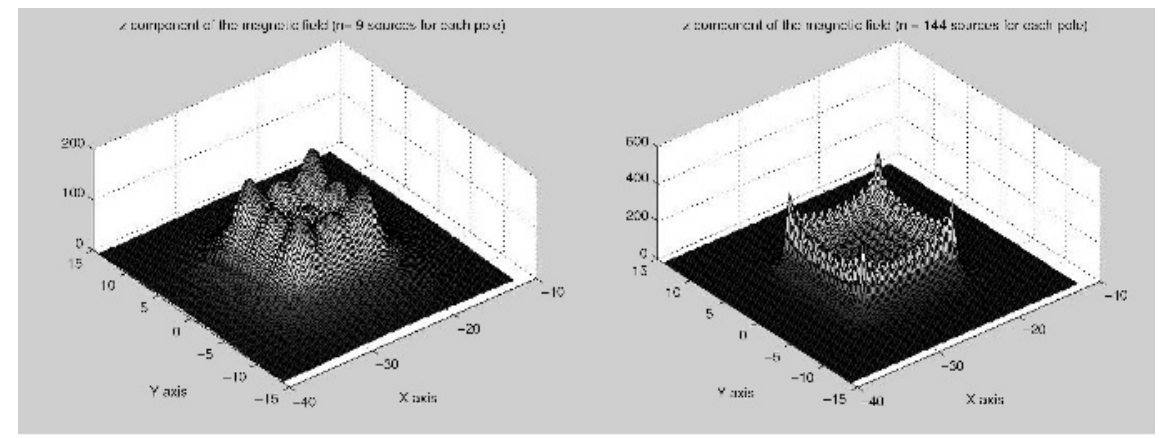

(a)

(b)

Figure 1.15 Normal component of the magnetic field on north pole for (a) 9 and (b) 144 sources. 


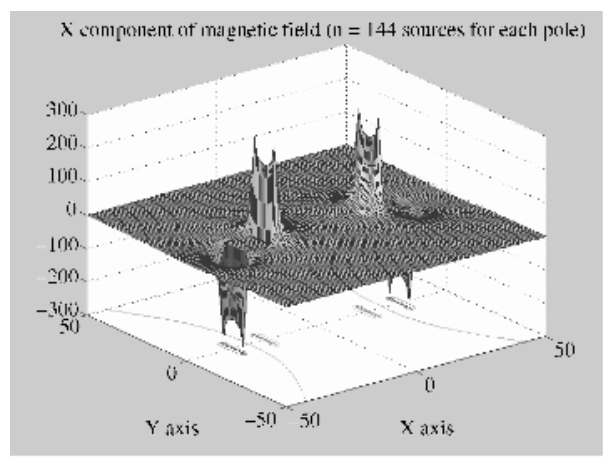

(a)

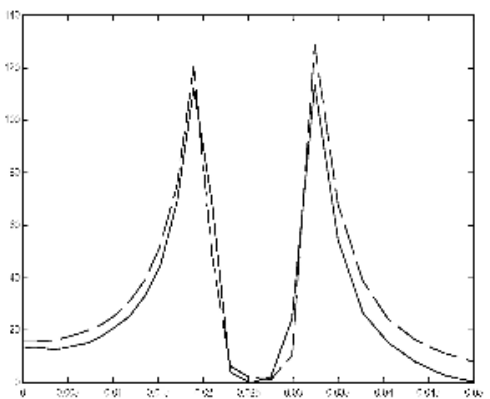

(b)

Figure 1.16 (a) Tangential component of the magnetic field computed by DPSM; (b) comparison of results obtained from DPSM (dashed line) and ANSYS (continuous line) simulation.

scalar condition is matched, corresponds to different components of the wave. This interesting point can be easily observed in ultrasonic problems. In ultrasonic modeling (discussed below) like magnetic problems, the DPSM synthesis can be carried out by matching scalar boundary conditions (pressure). In the ultrasonic modeling pressure, matching induces a variation for the vector terms (velocity). This point is illustrated in Figure 1.17. It should be noted here that if the continuity conditions must be satisfied on vectors (see examples on ultrasonic problems in Chapter 4 and electromagnetic problems in Chapter 6) instead of scalars, then triplet point sources must be used instead of simple point sources.

For all neighboring points j: $P_{j} \approx P_{i}+\frac{\overrightarrow{\partial P_{i}}}{\partial r_{i}} \times \overrightarrow{d r_{i j}}+0$

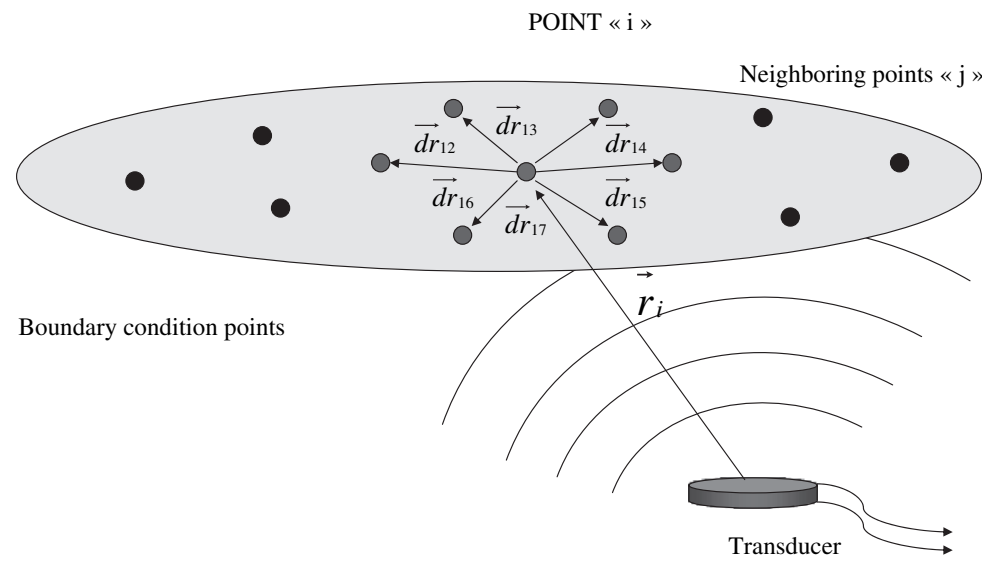

Figure 1.17 Field synthesis properties and boundary conditions. 
It will be shown in subsequent developments that for some boundary value problems, the boundary conditions may be specified on vector quantities. A simple such example is the ultrasonic transducer modeling. Ultrasonic transducer surfaces usually have a normal vibration speed, which induces a model in which the velocity vector is normal to the transducer surface. The next applications of DPSM will be illustrated with examples taken from the ultrasonic transducer modeling problems. For ultrasonic problems the point sources can be single elemental point source or triplet source. Although single elemental point sources are capable of satisfying only one boundary condition at a point, the triplet sources are capable of satisfying three boundary conditions ( $x, y$, and $z$ components of the velocity vector, for example) at one point.

\subsection{EXAMPLES FROM ULTRASONIC TRANSDUCER MODELING}

Most derivations on ultrasonic transducer modeling presented in this section are taken from Placko and Kundu (2004). For step by step development of DPSM formulation for ultrasonic problems, readers are referred to Chapter 3 of this book, where the formulation has been derived starting from the basic equations of ultrasonic problems.

Three most common ultrasonic wave fronts that are often used for modeling purposes are spherical, cylindrical, and plane. Spherical waves are generated by a point source in an infinite medium, cylindrical waves are generated by a line source, whereas plane waves are generated by an infinite plane, as shown in Figure 1.18.

These waves can be harmonic or nonharmonic. Harmonic waves are generated from harmonic (time dependence $=e^{-i \omega t}$ ) sources. The equation of the propagating spherical wave generated by a harmonic point source in a fluid space is given by (Kundu, 2004)

$$
G(r)=\frac{e^{i k_{\mathrm{f}} r}}{4 \pi r}
$$

and the equation of a propagating plane wave in a fluid is given by (Kundu, 2004)

$$
G\left(x_{1}\right)=e^{i k_{\mathrm{f}} x_{1}}
$$
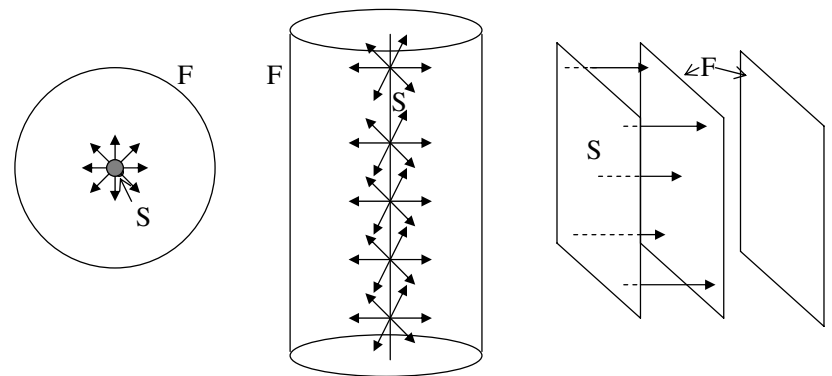

Figure 1.18 Point source (left), line source (middle) and infinite plane source (right) generating spherical, cylindrical and plane wave fronts, respectively. Sources are denoted by S and the wave fronts by $\mathrm{F}$. 
where, $k_{\mathrm{f}}\left(=\omega / c_{\mathrm{f}}\right)$ is the wave number of the fluid and is defined as the ratio of the angular frequency $(\omega)$ and the acoustic wave speed $\left(c_{f}\right)$ in the fluid, $r$ is the radial distance of the spherical wave front from the point source, and $x_{1}$ is the propagation distance of the plane wave front from the plane source. In Eqs. (1.13a) and (1.13b) $G$ can be either pressure $(p)$ or wave potential $(\phi)$. The wave potential-pressure relation is given by

$$
p=\rho \omega^{2} \phi
$$

If the wave sources of Figure 1.18 are located in a homogeneous solid instead of the fluid medium, then only compressional waves are generated in the solid, and their expressions can be obtained by simply substituting $k_{\mathrm{f}}$ by $k_{\mathrm{P}}$, where $k_{\mathrm{P}}$ is the $\mathrm{P}$-wave number of the solid. In the absence of any interface or boundary, the mode conversion does not occur and shear waves are not generated from the compressional waves.

In many nondestructive evaluation (NDE) applications, elastic waves are generated by a source of finite dimension and the wave fronts are not spherical or cylindrical or plane. Diameters of the commercially available ultrasonic transducers that are most commonly used in NDE for ultrasonic wave generation vary from a quarter of an inch to one inch. Of course, in special applications the ultrasonic sources can be much smaller (in the order of microns for high-frequency acoustic microscopy applications) or much larger (several inches for large-structure inspection). To correctly predict the ultrasonic field (displacement, stress, and pressure fields), generated by such finite sources, a semi-analytical modeling technique such as DPSM is needed.

\subsubsection{Justification of modeling a finite plane source by a distribution of point sources}

The pressure field due to a finite plane source can be assumed to be the summation of pressure fields generated by a number of point sources distributed over the finite source area as shown in Figure 1.19. The finite source can be, for example, the front face of a transducer as shown in this figure.

This assumption can be justified in the following manner:

A harmonic point source that expands and contracts alternately can be represented by a point and a sphere as shown in Figure 1.20a. The point represents the contracted position and the sphere (circle in a 2D figure) represents the expanded position. When a large number of these point sources are placed side by side on a plane surface, then

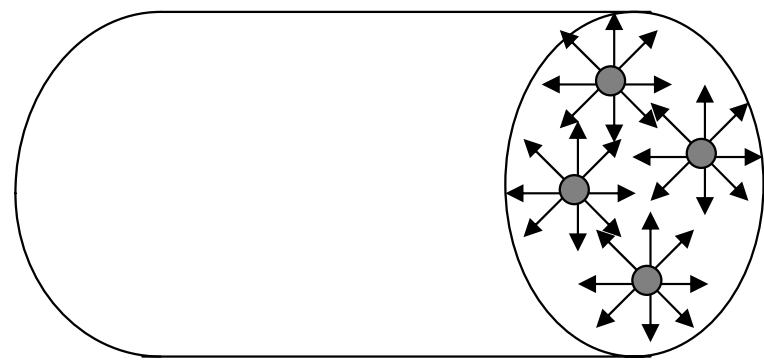

Figure 1.19 Four point sources distributed over a finite source 


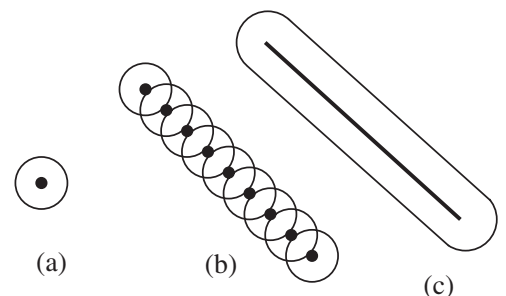

Figure 1.20 Contracted (dark) and expanded (thin line) positions of the particles for (a) a point source, (b) distributed finite number of point sources, and (c) a very large number of point sources.

the contracted and expanded positions of the point sources are shown in Figure 1.20b. The combined effect of a large number of point sources, placed side by side, is shown in Figure 1.20c, where the contracted (dark line) and expanded (thin line) positions of a line source or the cross section of a plane source are seen.

From Figure 1.20 it is clear that the combined effect of a large number of point sources distributed on a plane surface is the vibration of the particles in the direction normal to the plane surface. Nonnormal components of motion at a point on the surface, generated by neighboring source points, cancel each other. However, nonnormal components do not vanish along the edge of the surface. Therefore, the particles not only vibrate normal to the surface but also expand to a hemisphere and contract to the point along the edge, as shown in Figure 1.20c. If this edge effect does not have a significant contribution on the total motion, then the normal vibration of a finite plane surface can be approximately modeled by replacing the finite surface by a large number of point sources distributed over the surface.

\subsubsection{Planar piston transducer in a fluid}

The pressure field in a fluid for the planar piston transducer of finite diameter, as shown in Figure 1.21, is computed first. This problem can be solved in two ways as described below.

1.3.2.1 Conventional surface integral technique If one distributes the point sources over the transducer face, as discussed in Section 2.1, then the pressure field

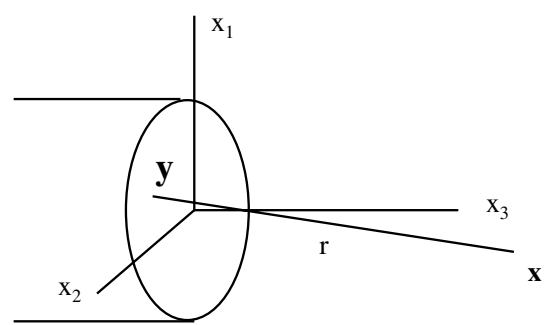

Figure 1.21 Point source $\mathrm{y}$ is on the transducer face, point $\mathbf{x}$ is where ultrasonic field is computed 
at position $\mathbf{x}$ in the fluid, due to the point sources at position $\mathbf{y}$ distributed over the transducer surface, can be given by integrating Eq. (1.13) over the transducer surface.

$$
p(\mathbf{x})=\int_{S} B \frac{\exp \left(i k_{\mathrm{f}} r\right)}{4 \pi r} d S(\mathbf{y})
$$

where $B$ is proportional to the source velocity amplitude. The above integral can be written in the following summation form:

$$
p(\mathbf{x})=\sum_{m=1}^{N}\left(\frac{B}{4 \pi} \Delta S_{m}\right) \frac{\exp \left(i k_{\mathrm{f}} r_{m}\right)}{r_{m}}=\sum_{m=1}^{N} A_{m} \frac{\exp \left(i k_{\mathrm{f}} r_{m}\right)}{r_{m}}
$$

However, from the Rayleigh-Sommerfield theory (Schmerr, 1998),

$$
p(\mathbf{x})=-\frac{i \omega \rho}{2 \pi} \int_{S} v_{3}(\mathbf{y}) \frac{\exp \left(i k_{\mathrm{f}} r\right)}{r} d S(\mathbf{y})
$$

where $v_{3}(\mathbf{y})$ is the particle velocity component normal to the transducer surface; note that $v_{1}(\mathbf{y})=v_{2}(\mathbf{y})=0$. For constant velocity of the transducer surface $\left(v_{3}=v_{0}\right)$, Eq. (1.15) is simplified to

$$
p(\mathbf{x})=-\frac{i \omega \rho v_{0}}{2 \pi} \int_{S} \frac{\exp \left(i k_{\mathrm{f}} r\right)}{r} d S(\mathbf{y})
$$

A comparison between Eqs. (1.14a) and (1.16) gives

$$
B=-2 i \omega \rho v_{0}
$$

Eq. (1.16) can be evaluated in closed form for a circular transducer of radius $a$ for the following two special cases (Schmerr, 1998):

(1) when $\mathbf{x}$ is located on the $x_{3}$-axis

$$
p\left(x_{3}\right)=\rho c_{\mathrm{f}} v_{0}\left\lfloor\exp \left(i k_{\mathrm{f}} x_{3}\right)-\exp \left(i k_{\mathrm{f}} \sqrt{x_{3}^{2}+a^{2}}\right)\right\rfloor
$$

(2) when $\mathbf{x}$ is in the far field. In other words, when $r$ is much greater than the transducer radius

$$
p\left(x_{1}, x_{2}, x_{3}\right)=-i \omega \rho v_{0} a^{2} \frac{\exp \left(i k_{\mathrm{f}} R\right)}{R} \frac{J_{1}\left(k_{\mathrm{f}} a \sin \theta\right)}{k_{\mathrm{f}} a \sin \theta}
$$

$R$ and $\theta$ of Eqs. (1.16b) and (1.16c) are shown in Figure 1.22. $J_{1}$ is the Bessel function of the first kind. 


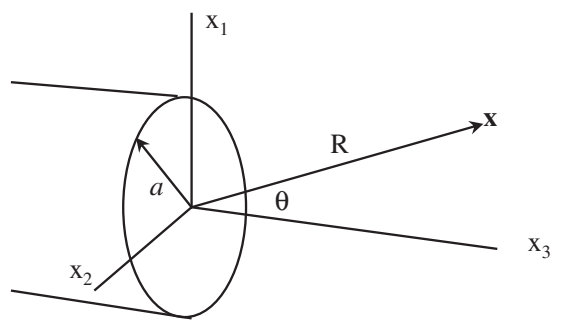

Figure 1.22 $R$ and $\theta$ denote the far field point $\mathbf{x}$

1.3.2.2 Alternative DPSM for computing the ultrasonic field An alternative technique to compute the strength of the distributed point sources on the transducer surface is given in this section.

Let the strength of the $m$ th point source be $A_{m}$ such that the pressure at a distance $r_{m}$ from the point source is given by Eq. (1.17) (also see Eq. (1.14b)).

$$
p_{m}(r)=A_{m} \frac{\exp \left(i k_{\mathrm{f}} r_{m}\right)}{r_{m}}
$$

If there are $N$ point sources distributed over the transducer surface, as shown in Figure 1.23, then the total pressure at point $\mathbf{x}$ is given by

$$
p(\mathbf{x})=\sum_{m=1}^{N} p_{m}\left(r_{m}\right)=\sum_{m=1}^{N} A_{m} \frac{\exp \left(i k_{\mathrm{f}} r_{m}\right)}{r_{m}}
$$

where $r_{m}$ is the distance of the $m$ th point source from point $\mathbf{x}$. Note that Eqs. (1.18) and (1.14b) are identical.

From the pressure-velocity relation, it is possible to compute the velocity at $\mathbf{x}$.

$$
-\frac{\partial p}{\partial n}=\rho \frac{\partial v_{n}}{\partial t}= \pm i \omega \rho v_{n}
$$

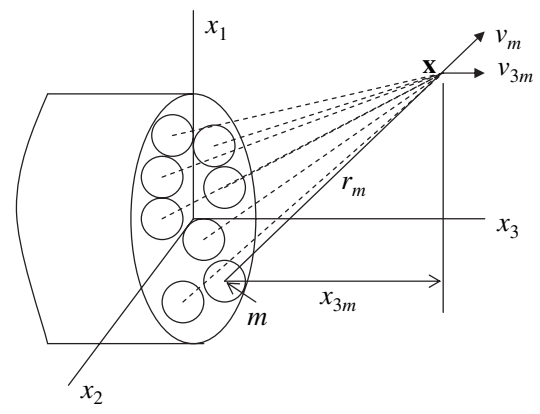

Figure 1.23 Velocity $v_{m}$ at point $\mathbf{x}$ due to the $m$-th point source 
Note that for $e^{ \pm i \omega t}$ time dependence of velocity, its derivative can be obtained by simply multiplying $v_{n}$ by positive or negative $i \omega$. For $e^{-i \omega t}$ time dependence,

$$
v_{n}=\frac{1}{i \omega \rho} \frac{\partial p}{\partial n}
$$

Therefore, the velocity in the radial direction, at a distance $r$ from the $m$ th point source, is given by

$$
\begin{aligned}
v_{m}(r) & =\frac{A_{m}}{i \omega \rho} \frac{\partial}{\partial r}\left(\frac{\exp \left(i k_{\mathrm{f}} r\right)}{r}\right)=\frac{A_{m}}{i \omega \rho}\left(\frac{i k_{\mathrm{f}} \exp \left(i k_{\mathrm{f}} r\right)}{r}-\frac{\exp \left(i k_{\mathrm{f}} r\right)}{r^{2}}\right) \\
& =\frac{A_{m}}{i \omega \rho} \frac{\exp \left(i k_{\mathrm{f}} r\right)}{r}\left(i k_{\mathrm{f}}-\frac{1}{r}\right)
\end{aligned}
$$

and the $x_{3}$ component of the velocity is

$$
v_{3 m}(r)=\frac{A_{m}}{i \omega \rho} \frac{\partial}{\partial x_{3}}\left(\frac{\exp \left(i k_{\mathrm{f}} r\right)}{r}\right)=\frac{A_{m}}{i \omega \rho} \frac{x_{3} \exp \left(i k_{\mathrm{f}} r\right)}{r^{2}}\left(i k_{\mathrm{f}}-\frac{1}{r}\right)
$$

When contributions of all $N$ sources are added, see Figure 1.23, then the total velocity in the $x_{3}$ direction at point $\mathbf{x}$ is obtained.

$$
v_{3}(\mathbf{x})=\sum_{m=1}^{N} v_{3 m}\left(r_{m}\right)=\sum_{m=1}^{N} \frac{A_{m}}{i \omega \rho} \frac{x_{3 m} \exp \left(i k_{\mathrm{f}} r_{m}\right)}{r_{m}^{2}}\left(i k_{\mathrm{f}}-\frac{1}{r_{m}}\right)
$$

where $x_{3 m}$ is the $x_{3}$ value measured from the $m$ th source as shown in Figure 1.23.

If the transducer surface velocity in the $x_{3}$ direction is given by $v_{0}$, then for all $\mathbf{x}$ values on the transducer surface, the velocity in the $\mathrm{x}_{3}$ direction should be equal to $v_{0}$. Therefore,

$$
v_{3}(\mathbf{x})=\sum_{m=1}^{N} \frac{A_{m}}{i \omega \rho} \frac{x_{3 m} \exp \left(i k_{\mathrm{f}} r_{m}\right)}{r_{m}^{2}}\left(i k_{\mathrm{f}}-\frac{1}{r_{m}}\right)=v_{0}
$$

By taking $N$ points on the transducer surface, it is possible to obtain a system of $N$ linear equations to solve for $N$ unknowns $\left(A_{1}, A_{2}, A_{3}, \ldots, A_{N}\right)$. However, difficulty arises when the point source location and the point of interest, $\mathbf{x}$, coincide because then $r_{m}$ becomes zero and $v_{3 m}$, from Eq. (1.24), becomes unbounded. Note that if point sources and points of interest $\mathbf{x}$ are both located on the transducer surface, only then these two points may coincide and $r_{m}$ can be zero. To avoid this possibility, the point sources are placed slightly behind the transducer surface as shown in Figure 1.24. For this arrangement the smallest value that $r_{m}$ can take is $r_{S}$.

When point $\mathbf{x}$ is located on the transducer surface as shown in Figure 1.24, then its $x_{3}$ component of velocity is matched with the transducer surface velocity $v_{0}$. In Figure 1.24 one can see that point $\mathbf{x}$ is located at the apex of the small spheres touching the transducer surface and the point sources are placed at the centers of these small 


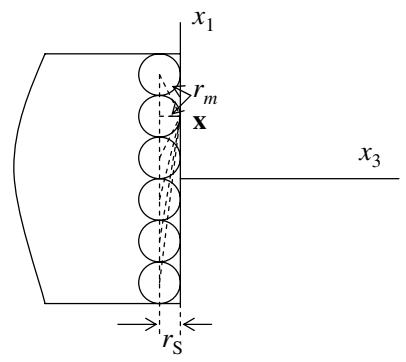

Figure 1.24 Point sources are located at $x_{3}=-r_{S}$ while the transducer surface is at $x_{3}=0$.

spheres. In addition to matching the $v_{3}$ component to $v_{0}$, if one wants to equate the other two components $v_{1}$ and $v_{2}$ to zero, then for each point $\mathbf{x}$ on the transducer surface, there are a total of three conditions or equations to be satisfied, as shown in Eqs. (1.24) and (1.25).

$$
\begin{aligned}
& v_{1}(\mathbf{x})=\sum_{m=1}^{N} \frac{A_{m}}{i \omega \rho} \frac{x_{1 m} \exp \left(i k_{\mathrm{f}} r_{m}\right)}{r_{m}^{2}}\left(i k_{\mathrm{f}}-\frac{1}{r_{m}}\right)=0 \\
& v_{2}(\mathbf{x})=\sum_{m=1}^{N} \frac{A_{m}}{i \omega \rho} \frac{x_{2 m} \exp \left(i k_{\mathrm{f}} r_{m}\right)}{r_{m}^{2}}\left(i k_{\mathrm{f}}-\frac{1}{r_{m}}\right)=0
\end{aligned}
$$

Thus, from $N$ points on the sphere surfaces, $3 N$ equations are obtained. Therefore, we get more equations than unknowns. To get the same number of unknowns as equations, the number of unknowns can be increased from $N$ to $3 N$ by replacing each point source by a triplet source. A triplet source is a combination of three point sources with three different strengths put together as shown in Figure 1.25. All sources are placed on the same plane at $x_{3}=-r_{S}$ parallel to the transducer surface. The three point sources of each triplet are located at the three vertices of an isosceles triangle that are oriented randomly, as shown in Figure 1.25, to preserve the isotropic material properties and prevent any preferential orientation. Thus, by solving a system of $3 N$ linear equations (for triplet sources) or a system of $N$ linear equations (for simple point sources), the source strengths $A_{m}$ associated with all point sources can be obtained. After getting $A_{m}$, the pressure $p(\mathbf{x})$ can be calculated at any point

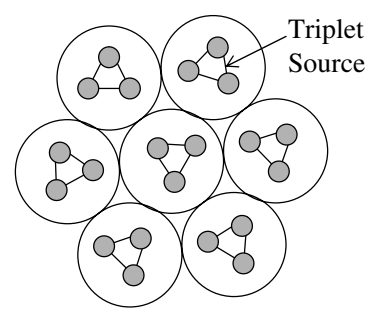

Figure 1.25 Randomly oriented triplet sources 
from Eq. (1.18), on the transducer surface or away. The pressure field obtained in this manner should be the same as that obtained from Eq. (1.16). Note that for a nonviscous perfect fluid, only the normal velocity component $\left(v_{3}\right)$ at the fluid-solid (transducer face) interface should be continuous. The velocity components parallel to the transducer face are not necessarily continuous because slippage may occur between the transducer face and the fluid. However, for viscous fluids such slippage is not possible and all the three velocity components should be continuous across the solid transducer face and fluid interface.

1.3.2.2.1 Matrix formulation The matrix formulation for computing the source strengths is given below. The following formulation is presented for triplet sources when all the three velocity components are matched at the transducer face and fluid interface. This is the case for viscous fluids. However, for nonviscous perfect fluids when only the normal velocity component needs to be matched, then simple elemental point sources should be used instead of triplet sources. Then $v_{1}$ and $v_{2}$ velocity components should be dropped from the following formulation. In that case the matrix and vector dimensions will be reduced from $3 N$ to $N$.

Eqs. (1.24) and (1.25) can be combined into the following matrix equation:

$$
\mathbf{V}_{\mathrm{S}}=\mathbf{M}_{\mathrm{SS}} \mathbf{A}_{\mathrm{S}}
$$

where $\mathbf{V}_{\mathrm{S}}$ is the $(3 N \times 1)$ vector of the velocity components at $N$ number of surface points $\mathbf{x}$, and $\mathbf{A}_{S}$ is the $(3 N \times 1)$ vector containing the strengths of $3 N$ number of point sources. $\mathbf{M}_{\mathrm{SS}}$ is the $(3 N \times 3 N)$ matrix relating the two vectors $\mathbf{V}_{\mathrm{S}}$ and $\mathbf{A}_{\mathrm{S}}$. From Eqs. (1.24) and (1.25) one can write

$$
\left\{\mathbf{V}_{\mathrm{S}}\right\}^{\mathrm{T}}=\left[\begin{array}{llllllllll}
v_{1}^{1} & v_{2}^{1} & v_{3}^{1} & v_{1}^{2} & v_{2}^{2} & v_{3}^{2} & \ldots & v_{1}^{N} & v_{2}^{N} & v_{3}^{N}
\end{array}\right]
$$

Note that the transpose of the column vector $\mathbf{V}_{\mathrm{S}}$ is a row vector of dimension $(1 \times 3 N)$. Elements of this vector are denoted by $v_{j}^{n}$, where the subscript $j$ can take values 1,2 , or 3 and indicate the direction of the velocity component. Superscript $n$ can take any value between 1 and $N$ corresponding to the point on the transducer surface at which the velocity component is defined.

For most ultrasonic transducers, $v_{j}^{n}=0$ for $j=1$ and 2 (the velocity component parallel to the transducer face) and $v_{j}^{n}=v_{0}$ for $j=3$ (the velocity component normal to the transducer face). Then, Eq. (1.25b) is simplified to

$$
\left\{\mathbf{V}_{\mathrm{S}}\right\}^{\mathrm{T}}=\left[\begin{array}{llllllllll}
0 & 0 & v_{0} & 0 & 0 & v_{0} & \ldots & 0 & 0 & v_{0}
\end{array}\right]
$$

Vector $\mathbf{A}_{\mathrm{S}}$ of the source strengths is given by

$$
\left\{\mathbf{A}_{\mathrm{S}}\right\}^{\mathrm{T}}=\left[\begin{array}{llllllllll}
A_{1} & A_{2} & A_{3} & A_{4} & A_{5} & A_{6} & \ldots & A_{(3 N-2)} & A_{(3 N-1)} & A_{3 N}
\end{array}\right]
$$

Note that the upper limits of Eqs. (1.24) and (1.25) are changed from $N$ to $3 N$ when triplet sources are considered, because then for every small sphere three point sources exist. Therefore, for $N$ spheres $3 N$ sources exist as shown in Figure 1.25. 
Finally, the square matrix $\mathbf{M}_{\mathrm{SS}}$ is obtained from Eqs. (1.24) and (1.25).

$$
\begin{aligned}
& \mathbf{M}_{\mathrm{SS}}= \\
& {\left[\begin{array}{cccccccc}
f\left(x_{11}^{1}, r_{1}^{1}\right) & f\left(x_{12}^{1}, r_{2}^{1}\right) & f\left(x_{13}^{1}, r_{3}^{1}\right) & f\left(x_{14}^{1}, r_{4}^{1}\right) & \ldots & \ldots & f\left(x_{1(3 N-1)}^{1}, r_{(3 N-1)}^{1}\right) & f\left(x_{1(3 N)}^{1}, r_{3 N}^{1}\right) \\
f\left(x_{21}^{1}, r_{1}^{1}\right) & f\left(x_{22}^{1}, r_{2}^{1}\right) & f\left(x_{23}^{1}, r_{3}^{1}\right) & f\left(x_{24}^{1}, r_{4}^{1}\right) & \ldots & \ldots & f\left(x_{2(3 N-1)}^{1}, r_{(3 N-1)}^{1}\right) & f\left(x_{2(3 N)}^{1}, r_{3 N}^{1}\right) \\
f\left(x_{31}^{1}, r_{1}^{1}\right) & f\left(x_{32}^{1}, r_{2}^{1}\right) & f\left(x_{33}^{1}, r_{3}^{1}\right) & f\left(x_{34}^{1}, r_{4}^{1}\right) & \ldots & \ldots & f\left(x_{3(3 N-1)}^{1}, r_{(3 N-1)}^{1}\right) & f\left(x_{3(3 N)}^{1}, r_{3 N}^{1}\right) \\
f\left(x_{11}^{2}, r_{1}^{2}\right) & f\left(x_{12}^{2}, r_{2}^{2}\right) & f\left(x_{13}^{2}, r_{3}^{2}\right) & f\left(x_{14}^{2}, r_{4}^{2}\right) & \ldots & \ldots & f\left(x_{1(3 N-1)}^{2}, r_{(3 N-1)}^{2}\right) & f\left(x_{1(3 N)}^{2}, r_{3 N}^{2}\right) \\
f\left(x_{21}^{2}, r_{1}^{2}\right) & f\left(x_{22}^{2}, r_{2}^{2}\right) & f\left(x_{23}^{2}, r_{3}^{2}\right) & f\left(x_{24}^{2}, r_{4}^{2}\right) & \ldots & \ldots & f\left(x_{2(3 N-1)}^{2}, r_{(3 N-1)}^{2}\right) & f\left(x_{2(3 N)}^{2}, r_{3 N}^{2}\right) \\
f\left(x_{31}^{2}, r_{1}^{2}\right) & f\left(x_{32}^{2}, r_{2}^{2}\right) & f\left(x_{33}^{2}, r_{3}^{2}\right) & f\left(x_{34}^{2}, r_{4}^{2}\right) & \ldots & \ldots & f\left(x_{3(3 N-1)}^{2}, r_{(3 N-1)}^{2}\right) & f\left(x_{3(3 N)}^{2}, r_{3 N}^{2}\right) \\
\ldots & \ldots & \ldots & \ldots & \ldots & \ldots & \ldots & \ldots \\
f\left(x_{31}^{N}, r_{1}^{N}\right) & f\left(x_{32}^{N}, r_{2}^{N}\right) & f\left(x_{33}^{N}, r_{3}^{N}\right) & f\left(x_{34}^{N}, r_{4}^{N}\right) & \ldots & \ldots & f\left(x_{3(3 N-1)}^{N}, r_{(3 N-1)}^{N}\right) & f\left(x_{3(3 N)}^{N}, r_{3 N}^{N}\right)
\end{array}\right]_{(3 N \times 3 N)}}
\end{aligned}
$$

where

$$
f\left(x_{j m}^{n}, r_{m}^{n}\right)=\frac{x_{j m}^{n} \exp \left(i k_{\mathrm{f}} r_{m}^{n}\right)}{i \omega \rho\left(r_{m}^{n}\right)^{2}}\left(i k_{\mathrm{f}}-\frac{1}{r_{m}^{n}}\right)
$$

In Eq. (1.25f), the first subscript $j$ of $x$ can take values 1, 2, or 3 and indicate whether $x$ is measured in the $x_{1}, x_{2}$, or $x_{3}$ direction. The subscript $m$ of $x$ and $r$ can take values from 1 to $3 N$ depending on which point source is considered, and the superscript $n$ can take any value between 1 and $N$ corresponding to the point on the transducer surface where the velocity component is computed. As mentioned earlier in this formulation, from $3 N$ point sources, three boundary conditions on the velocity are satisfied at every point of the $N$ boundary points. However, for nonviscous fluids the slippage between the transducer surface and the adjacent fluid surface is possible. Therefore, it is not necessary to enforce the no-slip condition $\left(v_{1}=v_{2}=0\right)$ on the fluid particles that are adjacent to the transducer surface.

If point $\mathbf{x}$ in Figure 1.24 is denoted by $\mathbf{x}_{n}$, indicating that this point is located on the $n$th boundary point, then the position vector connecting the $m$ th point source and the $n$th boundary point is denoted by $r_{m}^{n}$, and its three components in $x_{1}, x_{2}$, and $x_{3}$ directions are $x_{j m}^{n}, j=1,2,3$, in Eqs. (1.25e) and (1.25f).

From Eq. (1.25a) one gets the point source strengths by inverting the matrix $\mathbf{M}_{\mathrm{Ss}}$.

$$
\mathbf{A}_{\mathrm{S}}=\left[\mathbf{M}_{\mathrm{SS}}\right]^{-1} \mathbf{V}_{\mathrm{S}}=\mathbf{N}_{\mathrm{SS}} \mathbf{V}_{\mathrm{S}}
$$

If point sources are located very close to the transducer surface $\left(r_{\mathrm{S}}\right.$ in Fig. 1.24 is small), then the point source strengths $\left(\mathbf{A}_{\mathbf{S}}\right)$ should be approximately equal to the source strengths on the transducer surface. From Eqs. (1.14) and (1.16) we get,

$$
A_{m}=\frac{B}{4 \pi} \Delta S_{m}=-\frac{2 i \omega \rho v_{0}}{4 \pi} \frac{S}{N}
$$


In Eq. (1.25h) $S$ is the transducer surface area. Note that this equation gives the same source strength for all values of $m$. Therefore, the vector $\mathbf{A}_{\mathrm{S}}$, obtained from Eq. (1.25h), should have the following form:

$$
\left\{\mathbf{A}_{\mathrm{S}}\right\}^{\mathrm{T}}=-\frac{i \omega \rho v_{0} S}{2 \pi N}\left[\begin{array}{llllll}
1 & 1 & 1 & \ldots & \ldots & 1
\end{array}\right]
$$

After getting the source strength vector $\mathbf{A}_{\mathrm{S}}$ from Eq. (1.25g) or (1.25i), the pressure $p(\mathbf{x})$ or velocity vector $\mathbf{V}(\mathbf{x})$ at any point (on the transducer surface or away) can be obtained from Eq. (1.18) (for pressure) or Eqs. (1.24) and (1.25) (for velocity components). If the points in the fluid where the pressure and velocity vector are to be computed are called observation points or target points, then the pressure and velocity components, at these observation or target points, are obtained from the following matrix relation:

$$
\begin{gathered}
\mathbf{P}_{\mathrm{T}}=\mathbf{Q}_{\mathrm{TS}} \mathbf{A}_{\mathrm{S}} \\
\mathbf{V}_{\mathrm{T}}=\mathbf{M}_{\mathrm{TS}} \mathbf{A}_{\mathrm{S}}
\end{gathered}
$$

where $\mathbf{P}_{\mathrm{T}}$ is an $(M \times 1)$ vector containing pressure values at $M$ number of target points and $\mathbf{V}_{\mathrm{T}}$ is a $(3 M \times 1)$ vector containing three velocity components at every target point. The $\mathbf{V}_{\mathrm{T}}$ expression is similar to the $\mathbf{V}_{\mathrm{S}}$ expression given in Eq. (1.25b). The only difference is that its dimension is $(3 M \times 1)$ instead of $(3 N \times 1)$. Matrix $\mathbf{M}_{\mathrm{TS}}$ will be the same as $\mathbf{M}_{\mathrm{SS}}$ of Eq. (1.25e) if the target points are identical to the transducer surface points where the velocity components are matched to obtain the point source strength vector $\mathbf{A}_{\mathrm{S}}$ in Eq. (1.25g). However, for computing the velocity field at different points, the expression for $\mathbf{M}_{\mathrm{TS}}$ will be slightly different from the $\mathbf{M}_{\mathrm{SS}}$ expression given in Eq. (1.25e). Then its dimension will be $(3 M \times 3 N)$ as shown below:

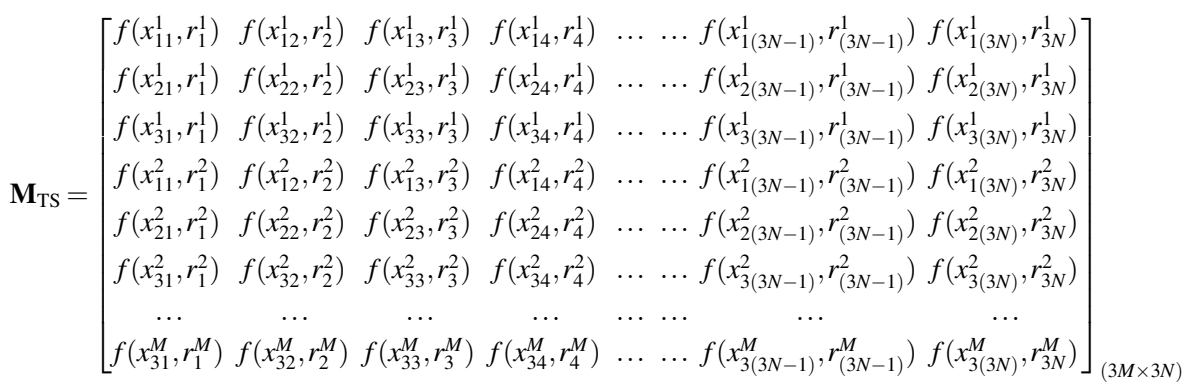

where $f\left(x_{j m}^{n}, r_{m}^{n}\right)$ is identical to the expression given in Eq. (1.25f). Definitions of the subscripts $j$ and $x$ do not change from those in Eq. (1.25f). The superscript $n$ of $x$ and $r$ can take any value between 1 and $M$ depending on which target point is considered. Note that $\mathbf{M}_{\mathrm{TS}}$ is not a square matrix when $M$ and $N$ are different. 
From Eq. (1.18) the matrix $\mathbf{Q}_{\mathrm{TS}}$ can be obtained when there are $3 N$ point sources and $M$ target points as shown in Eq. (1.251).

$$
\mathbf{Q}_{\mathrm{TS}}=\left[\begin{array}{cccccc}
\frac{\exp \left(i k_{\mathrm{f}} r_{1}^{1}\right)}{r_{1}^{1}} & \frac{\exp \left(i k_{\mathrm{f}} r_{2}^{1}\right)}{r_{2}^{1}} & \frac{\exp \left(i k_{\mathrm{f}} r_{3}^{1}\right)}{r_{3}^{1}} & \ldots & \ldots & \frac{\exp \left(i k_{\mathrm{f}} r_{3 N}^{1}\right)}{r_{3 N}^{1}} \\
\frac{\exp \left(i k_{\mathrm{f}} r_{1}^{2}\right)}{r_{1}^{2}} & \frac{\exp \left(i k_{\mathrm{f}} r_{2}^{2}\right)}{r_{2}^{2}} & \frac{\exp \left(i k_{\mathrm{f}} r_{3}^{2}\right)}{r_{3}^{2}} & \ldots & \ldots & \frac{\exp \left(i k_{\mathrm{f}} r_{3 N}^{2}\right)}{r_{3 N}^{2}} \\
\frac{\exp \left(i k_{\mathrm{f}} r_{1}^{3}\right)}{r_{1}^{3}} & \frac{\exp \left(i k_{\mathrm{f}} r_{2}^{3}\right)}{r_{2}^{3}} & \frac{\exp \left(i k_{\mathrm{f}} r_{3}^{3}\right)}{r_{3}^{3}} & \ldots & \ldots & \frac{\exp \left(i k_{\mathrm{f}} r_{3 N}^{3}\right)}{r_{3 N}^{3}} \\
\ldots & \ldots & \ldots & \ldots & \ldots \\
\frac{\exp \left(i k_{\mathrm{f}} r_{1}^{M}\right)}{r_{1}^{M}} & \frac{\exp \left(i k_{\mathrm{f}} r_{2}^{M}\right)}{r_{2}^{M}} & \frac{\exp \left(i k_{\mathrm{f}} r_{3}^{M}\right)}{r_{3}^{M}} & \ldots & \ldots & \frac{\exp \left(i k_{\mathrm{f}} r_{3 N}^{M}\right)}{r_{3 N}^{M}}
\end{array}\right]_{(M \times 3 N)}
$$

The definition of $r_{m}^{n}$ is identical for Eqs. (1.25k) and (1.25j); it is the distance between the $m$ th point source and $n$th target point.

This alternative method and matrix formulation, discussed here, for computing the ultrasonic field in a homogeneous fluid was first proposed by Placko and Kundu (2001); then it was extended to solve different ultrasonic problems by Placko et al. (2001, 2002) and Lee et al. (2002). This technique has been named by the authors as the distributed point source method or DPSM. The advantage of the DPSM technique is not obvious for this simple case of homogeneous medium. However, it will be evident later in this chapter when the ultrasonic field, in the presence of a finite inclusion or scatterer, will be computed.

Note that the DPSM technique, discussed in this section, is a general technique and is not restricted to the case of small value of $r_{\mathrm{S}}$ (see Fig. 1.24).

For small value of $r_{\mathrm{S}}$ Eq. (1.25i) can be used; otherwise, Eq. (1.25g) will have to be used. When Eq. (1.25g) is used, then Eq. (1.25j) is modified to

$$
\begin{aligned}
& \mathbf{P}_{\mathrm{T}}=\mathbf{Q}_{\mathrm{TS}} \mathbf{N}_{\mathrm{SS}} \mathbf{V}_{\mathrm{S}} \\
& \mathbf{V}_{\mathrm{T}}=\mathbf{M}_{\mathrm{TS}} \mathbf{N}_{\mathrm{SS}} \mathbf{V}_{\mathrm{S}}
\end{aligned}
$$

\section{Example 1.3.1}

Give the modified expressions for $\mathbf{V}_{\mathrm{S}}$ (Eq. (1.25c)) and $\mathbf{M}_{\mathrm{SS}}$ (Eq. (1.25e)) for the case when the triplet sources are replaced by single point sources, located at the centers of the small spheres (see Figs. 1.23 and 1.24), and only the normal displacement components (normal to the transducer surface) at the apex (or collocation points) on the transducer surface are equated to the transducer surface velocity $v_{0}$.

\section{Solution}

For $N$ number of spheres distributed over the transducer surface, there will be $N$ point sources and $N$ collocation points. Therefore, the velocity vector $\mathbf{V}_{\mathrm{S}}$ of Eq. (1.15c) will 
have $N$ entries instead of $3 N$ entries.

$$
\left\{\mathbf{V}_{\mathrm{S}}\right\}^{\mathrm{T}}=\left[\begin{array}{lllll}
v_{0} & v_{0} & v_{0} & \ldots \ldots & v_{0}
\end{array}\right]_{(N \times 1)}
$$

The matrix $\mathbf{M}_{\mathrm{SS}}$ of Eq. (1.25e) will have a dimension of $(N \times N)$ instead of $(3 N \times 3 N)$, because only the $v_{3}$ component is to be matched. The final form of $\mathbf{M}_{\mathrm{SS}}$ is given below.

$$
\begin{aligned}
& \mathbf{M}_{\mathrm{SS}}= \\
& {\left[\begin{array}{cccccccc}
f\left(x_{31}^{1}, r_{1}^{1}\right) & f\left(x_{32}^{1}, r_{2}^{1}\right) & f\left(x_{33}^{1}, r_{3}^{1}\right) & f\left(x_{34}^{1}, r_{4}^{1}\right) & \ldots & \ldots & f\left(x_{3(N-1)}^{1}, r_{(N-1)}^{1}\right) & f\left(x_{3 N}^{1}, r_{N}^{1}\right) \\
f\left(x_{31}^{2}, r_{1}^{2}\right) & f\left(x_{32}^{2}, r_{2}^{2}\right) & f\left(x_{33}^{2}, r_{3}^{2}\right) & f\left(x_{34}^{2}, r_{4}^{2}\right) & \ldots & \ldots & f\left(x_{3(N-1)}^{2}, r_{(3 N-1)}^{2}\right) & f\left(x_{3 N}^{2}, r_{N}^{2}\right) \\
f\left(x_{31}^{3}, r_{1}^{3}\right) & f\left(x_{32}^{3}, r_{2}^{3}\right) & f\left(x_{33}^{3}, r_{3}^{3}\right) & f\left(x_{34}^{3}, r_{4}^{3}\right) & \ldots & \ldots & f\left(x_{3(N-1)}^{3}, r_{(N-1)}^{3}\right) & f\left(x_{3 N}^{3}, r_{N}^{3}\right) \\
f\left(x_{31}^{4}, r_{1}^{4}\right) & f\left(x_{32}^{4}, r_{2}^{4}\right) & f\left(x_{33}^{4}, r_{3}^{4}\right) & f\left(x_{34}^{4}, r_{4}^{4}\right) & \ldots & \ldots & f\left(x_{3(N-1)}^{4}, r_{(3 N-1)}^{4}\right) & f\left(x_{3 N}^{4}, r_{N}^{4}\right) \\
\ldots & \ldots & \ldots & \ldots & \ldots & \ldots & \ldots & \ldots \\
\ldots & \ldots & \ldots & \ldots & \ldots & \ldots & \ldots & \ldots \\
\ldots & \ldots & \ldots & \ldots & \ldots & \ldots & \ldots & \ldots \\
f\left(x_{31}^{N}, r_{1}^{N}\right) & f\left(x_{32}^{N}, r_{2}^{N}\right) & f\left(x_{33}^{N}, r_{3}^{N}\right) & f\left(x_{34}^{N}, r_{4}^{N}\right) & \ldots & \ldots & f\left(x_{3(N-1)}^{N}, r_{(N-1)}^{N}\right) & f\left(x_{3 N}^{N}, r_{N}^{N}\right)
\end{array}\right]_{(N \times N)}}
\end{aligned}
$$

where, from Eq. (1.25f)

$$
f\left(x_{3 m}^{n}, r_{m}^{n}\right)=\frac{x_{3 m}^{n} \exp \left(i k_{\mathrm{f}} r_{m}^{n}\right)}{i \omega \rho\left(r_{m}^{n}\right)^{2}}\left(i k_{\mathrm{f}}-\frac{1}{r_{m}^{n}}\right)
$$

\section{Example 1.3.2}

For a large number of point sources distributed along the transducer surface, as shown in Figures 1.23 and 1.24, evaluate the source strength vector $\mathbf{A}_{\mathrm{S}}$ using Eq. (1.25g) for the $\mathbf{M}_{\mathrm{SS}}$ and $\mathbf{V}_{\mathrm{S}}$ expressions given in Eqs. (1.25o) and (1.25n), respectively.

\section{Solution}

For a large number of distributed point sources, the radius of the individual spheres becomes small (see Fig. 1.24). As the number of point sources approaches infinity, the radius of individual spheres reduces to zero. Therefore, $r_{m}^{n}$, the distance between the $m$ th point source and $n$th collocation point (or apex point), becomes zero for $m=n$. In other words, when the source is at the center of a sphere and the collocation point is at the apex of the same sphere, then the distance between the source and the collocation point is reduced to zero, as the number of point sources approaches infinity. 
Note that in Eq. (1.25p) $r_{m}^{n}$ appears in the denominator. Therefore, for small values of $r_{m}^{n}$ Eq. (1.25p) can be simplified in the following manner:

$f\left(x_{3 m}^{n}, r_{m}^{n}\right)=\frac{x_{3 m}^{n} \exp \left(i k_{\mathrm{f}} r_{m}^{n}\right)}{i \omega \rho\left(r_{m}^{n}\right)^{2}}\left(i k_{\mathrm{f}}-\frac{1}{r_{m}^{n}}\right) \approx \frac{x_{3 m}^{n} \exp \left(i k_{\mathrm{f}} r_{m}^{n}\right)}{i \omega \rho\left(r_{m}^{n}\right)^{2}}\left(-\frac{1}{r_{m}^{n}}\right)=-\frac{x_{3 m}^{n} \exp \left(i k_{\mathrm{f}} r_{m}^{n}\right)}{i \omega \rho\left(r_{m}^{n}\right)^{3}}$

Note that all spheres have the same radius $r_{m}=r_{\mathrm{S}}=r$; therefore, $x_{3 m}^{n}=r$. Substituting it into the above expression and expanding the exponential term in its series expansion,

$$
f\left(x_{3 m}^{n}, r_{m}^{n}\right) \approx-\frac{x_{3 m}^{n} \exp \left(i k_{\mathrm{f}} r_{m}^{n}\right)}{i \omega \rho\left(r_{m}^{n}\right)^{3}} \approx-\frac{r}{i \omega \rho\left(r_{m}^{n}\right)^{3}}\left(1+i k_{\mathrm{f}} r_{m}^{n}+\ldots\right) \approx-\frac{r}{i \omega \rho\left(r_{m}^{n}\right)^{3}}
$$

for $m=n, r_{m}^{n}=r_{m}^{m}=r$. Substituting it into Eq. (1.25q), we get (no summation on $m$ is implied)

$$
f\left(x_{3 m}^{m}, r_{m}^{m}\right) \approx-\frac{r}{i \omega \rho\left(r_{m}^{m}\right)^{3}} \approx-\frac{r}{i \omega \rho r^{3}} \approx-\frac{1}{i \omega \rho r^{2}}
$$

Substitution of Eqs. (1.25q) and (1.25r) into Eq. (1.25o) yields

$$
\mathbf{M}_{\mathrm{SS}}=-\frac{1}{i \omega \rho r^{2}}\left[\begin{array}{ccccc}
1 & \left(\frac{r}{r_{2}^{1}}\right)^{3} & \left(\frac{r}{r_{3}^{1}}\right)^{3} & \ldots & \left(\frac{r}{r_{N}^{1}}\right)^{3} \\
\left(\frac{r}{r_{1}^{2}}\right)^{3} & 1 & \left(\frac{r}{r_{3}^{2}}\right)^{3} & \ldots & \left(\frac{r}{r_{N}^{2}}\right)^{3} \\
\left(\frac{r}{r_{1}^{3}}\right)^{3} & \left(\frac{r}{r_{2}^{3}}\right)^{3} & 1 & \ldots & \left(\frac{r}{r_{N}^{3}}\right)^{3} \\
\cdots & \cdots & \cdots & \cdots & \cdots \\
\left(\frac{r}{r_{1}^{N}}\right)^{3} & \left(\frac{r}{r_{2}^{N}}\right)^{3} & \left(\frac{r}{r_{3}^{N}}\right)^{3} & \cdots & 1
\end{array}\right]_{(N \times N)}
$$

It should be noted here that for $m \neq n, r_{m}^{n}>r$. Therefore, in the above matrix expression, the off-diagonal terms are smaller than the diagonal terms. With an increasing number of point sources as $r$ approaches zero, all off-diagonal terms vanish and the above matrix simplifies to

$$
\mathbf{M}_{\mathrm{SS}}=-\frac{1}{i \omega \rho r^{2}}\left[\begin{array}{ccccc}
1 & 0 & 0 & \ldots & 0 \\
0 & 1 & 0 & \ldots & 0 \\
0 & 0 & 1 & \ldots & 0 \\
\ldots & \ldots & \ldots & \ldots & \ldots \\
0 & 0 & 0 & \ldots & 1
\end{array}\right]_{(N \times N)}
$$


Therefore, from Eq. (1.25g):

$\mathbf{A}_{\mathrm{S}}=\left[\mathbf{M}_{\mathrm{SS}}\right]^{-1} \mathbf{V}_{\mathrm{S}}=-i \omega \rho r^{2}\left[\begin{array}{ccccc}1 & 0 & 0 & \ldots & 0 \\ 0 & 1 & 0 & \ldots & 0 \\ 0 & 0 & 1 & \ldots & 0 \\ \ldots & \ldots & \ldots & \ldots & \ldots \\ 0 & 0 & 0 & \ldots & 1\end{array}\right]\left\{\begin{array}{c}v_{0} \\ v_{0} \\ v_{0} \\ \ldots \\ v_{0}\end{array}\right\}=-i \omega \rho v_{0} r^{2}\left\{\begin{array}{c}1 \\ 1 \\ 1 \\ \ldots \\ 1\end{array}\right\}$

\section{Example 1.3.3}

Prove that the coefficients of Eqs. (1.25i) and (1.25s) are identical.

\section{Solution}

The total surface area from $N$ hemispheres, associated with the $N$ point sources, is equated to the transducer surface area $S$. Therefore,

$$
\begin{aligned}
& S=2 \pi r^{2} \times N=2 \pi N r^{2} \\
& \Rightarrow r^{2}=\frac{S}{2 \pi N}
\end{aligned}
$$

Substituting it into the coefficient of Eq. (1.25s) gives

$$
-i \omega o v_{0} r^{2}=-\frac{i \omega o v_{0} S}{2 \pi N}
$$

1.3.2.3 Restrictions on $r_{S}$ for point source distribution It is evident from Figure 1.24 that as the number of point sources used to model the transducer surface is increased, $r_{S}$ is decreased. It is expected that with larger number of point sources, the computation time and accuracy both should increase. The question is what optimum number of point sources should produce reliable results? To answer this question the following analysis is carried out:

For a very small transducer of surface area $d S$ vibrating with a velocity of amplitude $v_{0}$ in the $x_{3}$ direction, the pressure at point $\mathbf{x}$ (at a distance $r$ from the source at point $\mathbf{y}$ ) can be computed from Eq. (1.16).

$$
p(\mathbf{x})=-\frac{i \omega \rho v_{0}}{2 \pi} \frac{\exp \left(i k_{\mathrm{f}} r\right)}{r} d S
$$

Using Eq. (1.20), the particle velocity in the radial direction can be computed from the above pressure field.

$$
\begin{aligned}
v_{r} & =\frac{1}{i \omega \rho} \frac{\partial p}{\partial r}=\frac{1}{i \omega \rho}\left(\frac{-i \omega \rho v_{0}}{2 \pi}\right)\left(\frac{i k_{\mathrm{f}} \exp \left(i k_{\mathrm{f}} r\right)}{r}-\frac{\exp \left(i k_{\mathrm{f}} r\right)}{r^{2}}\right) d S \\
& =-\frac{v_{0}\left(i k_{\mathrm{f}} r-1\right)}{2 \pi r^{2}} \exp \left(i k_{\mathrm{f}} r\right) d S
\end{aligned}
$$


and the velocity in the $x_{3}$ direction

$$
v_{3}=\frac{1}{i \omega \rho} \frac{\partial p}{\partial x_{3}}=\frac{1}{i \omega \rho} \frac{\partial p}{\partial r} \frac{\partial r}{\partial x_{3}}=-\frac{v_{0}\left(i k_{\mathrm{f}} r-1\right)}{2 \pi r^{2}} \exp \left(i k_{\mathrm{f}} r\right) d S \frac{x_{3}-y_{3}}{r}
$$

where $x_{3}$ and $y_{3}$ are the $x_{3}$ coordinate values of points $\mathbf{x}$ and $\mathbf{y}$, respectively.

If the point $\mathbf{x}$ is taken on the surface of the sphere of radius $r_{\mathrm{S}}$ as shown in Figure 1.24, then $r=r_{\mathrm{S}}=x_{3}-y_{3}$, and $v_{3}$ of Eq. (1.28) is simplified to

$$
\begin{aligned}
v_{3} & =-\frac{v_{0}\left(i k_{\mathrm{f}} r_{\mathrm{S}}-1\right)}{2 \pi r_{\mathrm{S}}^{2}} \exp \left(i k_{\mathrm{f}} r_{\mathrm{S}}\right) d S=v_{0}\left(1-i k_{\mathrm{f}} r_{\mathrm{S}}\right)\left(1+i k_{\mathrm{f}} r_{\mathrm{S}}+O\left(k_{\mathrm{f}}^{2} r_{\mathrm{S}}^{2}\right)\right) \frac{d S}{2 \pi r_{\mathrm{S}}^{2}} \\
& \approx v_{0}\left(1+k_{\mathrm{f}}^{2} r_{\mathrm{S}}^{2}\right) \frac{d S}{2 \pi r_{\mathrm{S}}^{2}}
\end{aligned}
$$

The right-hand side of Eq. (1.29) should be equal to $v_{0}$ because the pressure computed in Eq. (1.16) is obtained from the transducer surface velocity $v_{0}$ in the $x_{3}$ direction. Hence, the velocity at $\mathbf{x}$ when $\mathbf{x}$ is taken on the transducer surface should be equal to $v_{0}$. The right-hand side of Eq. (1.29) is $v_{0}$ when $d S=2 \pi r_{\mathrm{S}}^{2}$ and $k_{\mathrm{f}}^{2} r_{\mathrm{S}}^{2} \ll 1$. Therefore, $d S$ should be the surface area of a hemisphere of radius $r_{\mathrm{S}}$, and the second condition implies the following:

$$
\begin{aligned}
k_{\mathrm{f}}^{2} r_{\mathrm{S}}^{2} & =\left(\frac{2 \pi f}{c_{\mathrm{f}}} r_{\mathrm{S}}\right)^{2} \ll 1 . \\
& \Rightarrow r_{\mathrm{S}} \ll \frac{c_{\mathrm{f}}}{2 \pi f} \\
& \Rightarrow r_{\mathrm{S}} \ll \frac{\lambda_{\mathrm{f}}}{2 \pi}
\end{aligned}
$$

where $\lambda_{\mathrm{f}}$ is the wavelength in the fluid. Eq. (1.30) is used to compute the number of point sources in the following manner: Take a value of $r_{\mathrm{S}}$ satisfying the condition (1.30), then compute the number of point sources $N$ from the transducer surface area $S$ from the relation

$$
N=\frac{S}{2 \pi r_{\mathrm{S}}^{2}}
$$

Note that the spacing between two neighboring point sources is different from $r_{\mathrm{S}}$. If the point sources are arranged uniformly at the vertex points of squares of side length $a$, then each point source should be associated with an area of $a^{2}$ of the flat transducer face. This area is then equated to the hemispherical surface area of each point source to obtain

$$
\begin{aligned}
a^{2} & =2 \pi r_{\mathrm{S}}^{2} \\
& \Rightarrow a=r_{\mathrm{S}} \sqrt{2 \pi}
\end{aligned}
$$


Substituting Eq. (1.30) into the above equation we get

$$
\begin{aligned}
a & =r_{\mathrm{S}} \sqrt{2 \pi} \ll \sqrt{2 \pi} \frac{\lambda_{\mathrm{f}}}{2 \pi} \\
& \Rightarrow a \ll \frac{\lambda_{\mathrm{f}}}{\sqrt{2 \pi}}
\end{aligned}
$$

\subsubsection{Focused transducer in a homogeneous fluid}

For a focused transducer, as shown in Figure 1.26, the ultrasonic field in the fluid can be modeled by distributing the point sources along the curved transducer face. O'Neil (1949) argued that for transducers with small curvature the Rayleigh-Sommerfield integral representation (Eq. (1.16)) holds if the surface integral is carried out over the curved surface. Therefore, the DPSM technique, discussed in Section 1.3.2.2, holds good for the curved transducer face as well. In this case the point sources should be distributed over a curved surface, instead of a flat surface.

The integral representation of the pressure field in the fluid for a focused transducer should be the same as Eq. (1.16). This integral can be evaluated in closed form, for computing the pressure variation on the central axis of the transducer; in other words, for the on-axis pressure computation. The on-axis pressure field is given by (Schmerr, 1998)

$$
p\left(x_{3}\right)=\frac{\rho c v_{0}}{q_{0}}\left[\exp \left(i k_{\mathrm{f}} x_{3}\right)-\exp \left(i k_{\mathrm{f}} \sqrt{x_{3}^{2}+a^{2}}\right)\right]=\frac{\rho c v_{0}}{q_{0}}\left[\exp \left(i k_{\mathrm{f}} x_{3}\right)-\exp \left(i k_{\mathrm{f}} r_{e}\right)\right]
$$

where

$$
q_{0}=1-\frac{x_{3}}{R_{0}}
$$

$R_{0}$ is the radius of curvature of the transducer face, $r_{\mathrm{e}}$ is the distance of the point of interest from the transducer edge.

At the geometric focus point, $x_{3}=R_{0}$, the pressure is given by (Schmerr, 1998)

$$
p\left(R_{0}\right)=-i \rho c v_{0} k_{\mathrm{f}} h \exp \left(i k_{\mathrm{f}} R_{0}\right)
$$

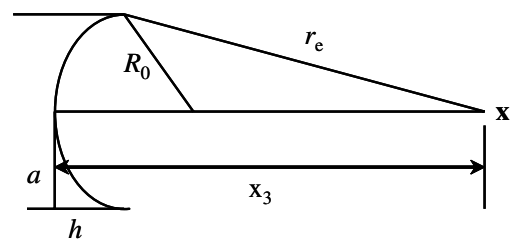

Figure 1.26 Focused transducer $-R_{0}$ is the radius of curvature of the transducer, $a$ is its radius, focal point is denoted as $\mathbf{x}$. 
If, at $x_{3}=z$, the on-axis pressure is maximum, then $z$ should satisfy the following equation (Schmerr, 1998):

$$
\cos \left(\frac{k_{\mathrm{f}} \delta}{2}\right)=\frac{2(\delta+z) \sin \left(k_{\mathrm{f}} \delta / 2\right)}{(\delta+h) q_{0} k_{\mathrm{f}} R_{0}}
$$

where

$$
\delta=r_{\mathrm{e}}-z=\left[(z-h)^{2}+a^{2}\right]^{\frac{1}{2}}-z
$$

\subsubsection{Ultrasonic field in a nonhomogeneous fluid in the presence of an interface}

If the fluid, in front of the transducer, is not homogeneous but is made of two fluids with an interface between the two, then the ultrasonic signal generated by the transducer will go through reflection and transmission at the interface as shown in Figure 1.27. In this case, the pressure field in fluid 1, at point $\mathrm{P}$, can be computed by adding the contributions of the direct incident ray $\left(\mathrm{R}_{1}\right)$ and reflected ray. To compute the pressure at point $\mathrm{Q}$ in fluid 2, the contribution of only the transmitted ray needs to be considered. Acoustic wave speed and density of the two fluids are denoted by $c_{\mathrm{f}}$ and $\rho_{\mathrm{f}}$ for fluid 1, and $c_{\mathrm{f} 2}$ and $\rho_{\mathrm{f} 2}$ for fluid 2, as shown in Figure 1.27.

In Figure 1.27, point $C$ is either on the transducer surface for Rayleigh-Sommerfield integral representation of the pressure field, or just behind the transducer surface (as shown in Figure 1.24) for the DPSM modeling, discussed in Section 1.2.2.2. We are interested in computing the acoustic pressure at point $\mathrm{P}$ in fluid 1 and at point $\mathrm{Q}$ in fluid 2. As shown in the figure, point $P$ receives a direct ray $\left(R_{1}\right)$ from point $C$ and a ray $\left(R_{3}\right)$ reflected by the interface at point $\mathrm{T}$. Point $\mathrm{Q}$ can only receive a ray from point $\mathrm{C}$ after it is transmitted at the interface at point T. Position vectors of points $\mathrm{C}, \mathrm{T}, \mathrm{P}$, and $\mathrm{Q}$ are denoted by $\mathbf{y}, \mathbf{z}, \mathbf{x}$, and $\mathbf{x}$, respectively, as shown in the figure. Because both points $P$ and $\mathrm{Q}$ are the points where the pressure field is to be computed, we use the same symbol $\mathbf{x}$ for denoting the positions of these two points although those are not at the same location.

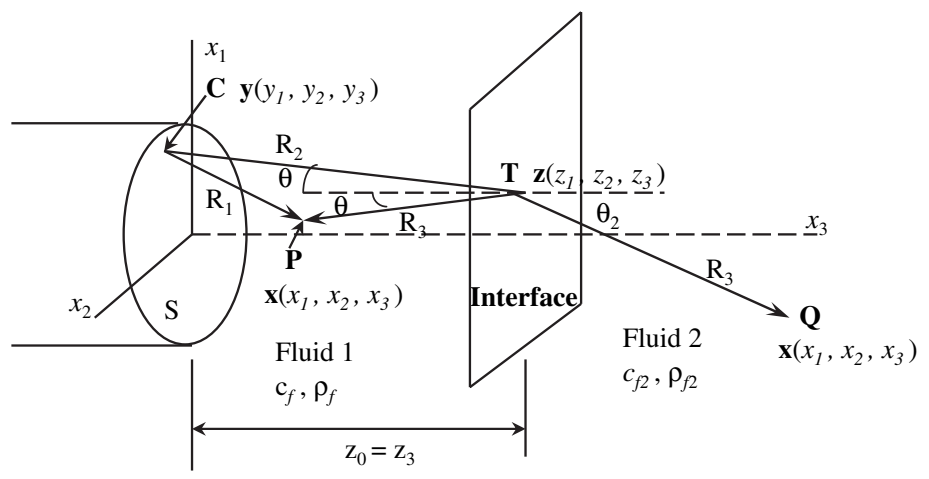

Figure 1.27 Transducer in front of an interface between two fluids of different properties 
Now, the question is, when the coordinates $\left(x_{1}, x_{2}, x_{3}\right)$ and $\left(y_{1}, y_{2}, y_{3}\right)$ are known, then how to obtain the coordinates $\left(\mathrm{z}_{1}, \mathrm{z}_{2}, \mathrm{z}_{3}\right)$ of $\mathrm{T}$ on the interface, where the ray is reflected or transmitted to reach point $\mathrm{P}$ or $\mathrm{Q}$. This question can be answered from geometric considerations, as given below.

1.3.4.1 Pressure field computation in fluid 1 at point $\boldsymbol{P}$ Let vectors $\underline{A}$ and $\underline{B}$ represent CT and TP, respectively, in Figure 1.27; then,

$$
\begin{aligned}
& \underline{A}=\left(z_{1}-y_{1}\right) \underline{e_{1}}+\left(z_{2}-y_{2}\right) \underline{e_{2}}+\left(z_{3}-y_{3}\right) \underline{e_{3}} \\
& \underline{B}=\left(x_{1}-z_{1}\right) \underline{e_{1}}+\left(x_{2}-z_{2}\right) \underline{e_{2}}+\left(x_{3}-z_{3}\right) \underline{e_{3}}
\end{aligned}
$$

Note that the magnitudes of vectors $\underline{A}$ and $\underline{B}$ are $R_{2}$ and $R_{3}$, respectively.

$$
\begin{aligned}
& R_{2}=\left\{\left(z_{1}-y_{1}\right)^{2}+\left(z_{2}-y_{2}\right)^{2}+\left(z_{3}-y_{3}\right)^{2}\right\}^{\frac{1}{2}} \\
& R_{3}=\left\{\left(x_{1}-z_{1}\right)^{2}+\left(x_{2}-z_{2}\right)^{2}+\left(x_{3}-z_{3}\right)^{2}\right\}^{\frac{1}{2}}
\end{aligned}
$$

Unit vectors $\hat{A}=\frac{\underline{A}}{R_{2}}$ and $\hat{B}=\frac{\underline{B}}{R_{3}}$

Unit vector $\hat{n}$ normal to the interface is given by

$\hat{n}=\left\{\begin{array}{l}0 \\ 0 \\ 1\end{array}\right\}$. Note that for an inclined interface, $\hat{n}=\left\{\begin{array}{l}n_{1} \\ n_{2} \\ n_{3}\end{array}\right\}$

From the problem geometry one can easily see that

$$
\begin{array}{r}
\hat{n} \times \hat{A}=\hat{n} \times \hat{B} \\
\hat{n} \cdot \hat{A}=-\hat{n} \cdot \hat{B}
\end{array}
$$

Let

$$
\hat{A}=\left\{\begin{array}{l}
a_{1} \\
a_{2} \\
a_{3}
\end{array}\right\}, \quad \hat{B}=\left\{\begin{array}{l}
b_{1} \\
b_{2} \\
b_{3}
\end{array}\right\}
$$

Substituting the above unit vector expressions in Eq. (1.38) one gets

$$
\operatorname{det}\left[\begin{array}{lll}
e_{1} & e_{2} & e_{3} \\
n_{1} & n_{2} & n_{3} \\
a_{1} & a_{2} & a_{3}
\end{array}\right]=\operatorname{det}\left[\begin{array}{lll}
e_{1} & e_{2} & e_{3} \\
n_{1} & n_{2} & n_{3} \\
b_{1} & b_{2} & b_{3}
\end{array}\right]
$$

or in an alternate representation

$$
\left[\begin{array}{ccc}
0 & -n_{3} & n_{2} \\
n_{3} & 0 & -n_{1} \\
-n_{2} & n_{1} & 0
\end{array}\right]\left\{\begin{array}{l}
a_{1} \\
a_{2} \\
a_{3}
\end{array}\right\}=\left[\begin{array}{ccc}
0 & -n_{3} & n_{2} \\
n_{3} & 0 & -n_{1} \\
-n_{2} & n_{1} & 0
\end{array}\right]\left\{\begin{array}{l}
b_{1} \\
b_{2} \\
b_{3}
\end{array}\right\}
$$


or

$$
\left[\begin{array}{ccc}
0 & -1 & 0 \\
-1 & 0 & 0 \\
0 & 0 & 0
\end{array}\right]\left\{\begin{array}{l}
a_{1} \\
a_{2} \\
a_{3}
\end{array}\right\}=\left[\begin{array}{ccc}
0 & -1 & 0 \\
-1 & 0 & 0 \\
0 & 0 & 0
\end{array}\right]\left\{\begin{array}{l}
b_{1} \\
b_{2} \\
b_{3}
\end{array}\right\}
$$

or

$$
\left\{\begin{array}{c}
-a_{2} \\
a_{1} \\
0
\end{array}\right\}=\left\{\begin{array}{c}
-b_{2} \\
b_{1} \\
0
\end{array}\right\}
$$

or

$$
\left\{\begin{array}{c}
-\frac{z_{2}-y_{2}}{R_{2}} \\
\frac{z_{1}-y_{1}}{R_{2}}
\end{array}\right\}=\left\{\begin{array}{c}
\frac{z_{2}-x_{2}}{R_{3}} \\
-\frac{z_{1}-x_{1}}{R_{3}}
\end{array}\right\}
$$

Similarly from Eq. (1.39)

$$
\left[\begin{array}{lll}
n_{1} & n_{2} & n_{3}
\end{array}\right]\left\{\begin{array}{l}
a_{1} \\
a_{2} \\
a_{3}
\end{array}\right\}=-\left[\begin{array}{lll}
n_{1} & n_{2} & n_{3}
\end{array}\right]\left\{\begin{array}{l}
b_{1} \\
b_{2} \\
b_{3}
\end{array}\right\}
$$

or

$$
\left[\begin{array}{lll}
0 & 0 & 0
\end{array}\right]\left\{\begin{array}{l}
a_{1} \\
a_{2} \\
a_{3}
\end{array}\right\}=-\left[\begin{array}{lll}
0 & 0 & 0
\end{array}\right]\left\{\begin{array}{l}
b_{1} \\
b_{2} \\
b_{3}
\end{array}\right\}
$$

or

$$
a_{3}=-b_{3} \Rightarrow \frac{z_{3}-y_{3}}{R_{2}}=\frac{z_{3}-x_{3}}{R_{3}}
$$

Solving the above equations

$$
\begin{aligned}
& z_{1}=\frac{y_{1}\left(x_{3}-z_{3}\right)-x_{1}\left(z_{3}-y_{3}\right)}{x_{3}-2 z_{3}+y_{3}} \\
& z_{2}=\frac{y_{2}\left(x_{3}-z_{3}\right)-x_{2}\left(z_{3}-y_{3}\right)}{x_{3}-2 z_{3}+y_{3}}
\end{aligned}
$$

Note that if point $\mathrm{C}$ is on the $x_{1} x_{2}$ plane, then $y_{3}=0$, and for fluid $1, x_{3}$ is between 0 and $z_{3}$; therefore, the denominator of Eq. (1.43) should never become zero. After obtaining $z_{1}$ and $z_{2}$, from Eq. (1.43), the lengths $R_{2}$ and $R_{3}$ can be easily obtained from Eq. (1.37). To evaluate $R_{1}$, one does not need $z_{1}$ and $z_{2}$. It is simply equal to

$$
R_{1}=\left\{\left(x_{1}-y_{1}\right)^{2}+\left(x_{2}-y_{2}\right)^{2}+\left(x_{3}-y_{3}\right)^{2}\right\}^{\frac{1}{2}}
$$


Then the pressure field at point $\mathrm{P}$ can be obtained from the following equation:

$$
p_{\mathrm{P}}(x)=-\frac{i \omega \rho_{\mathrm{f}} v_{o}}{2 \pi} \int_{\mathrm{S}} \frac{\exp \left(i k_{\mathrm{f}} R_{1}\right)}{R_{1}} d S-\frac{i \omega \rho_{\mathrm{f}} v_{o}}{2 \pi} \int_{\mathrm{S}} \frac{R \cdot \exp \left\{i k_{\mathrm{f}}\left(R_{2}+R_{3}\right)\right\}}{R_{2}+R_{3}} d S
$$

In Eq. (1.45) the first integral corresponds to the wave path $\mathrm{CP}$ and the second integral corresponds to the wave path CTP. Note that both these integrals are similar to the expression given in Eq. (1.16); the only difference is that in the second integral expression, the reflection coefficient $R$ has been included because this wave reaches point $\mathrm{P}$ after being reflected at the interface. The expression of the reflection coefficient $R$ is given in Eq. (1.208) of Kundu (2004).

$$
R=\frac{\rho_{2} c_{\mathrm{f} 2} \cos \theta_{1}-\rho_{1} c_{\mathrm{f} 1} \cos \theta_{2}}{\rho_{2} c_{\mathrm{f} 2} \cos \theta_{1}+\rho_{1} c_{\mathrm{f} 1} \cos \theta_{2}}
$$

In this case, the incident angle is $\theta$, transmitted angle is $\theta_{2}$, fluid densities are $\rho_{\mathrm{f}}$ and $\rho_{\mathrm{f} 2}$, and acoustic wave speeds in the two fluids are $c_{\mathrm{f}}$ and $c_{\mathrm{f} 2}$. Then, the transmitted angle $\theta_{2}$ can be expressed in terms of the incident angle $\theta$ using Snell's law (see Eq. (1.204) of Kundu (2004)).

$$
\cos \theta_{2}=\sqrt{1-\sin ^{2} \theta_{2}}=\sqrt{1-\left(\frac{c_{\mathrm{f} 2} \sin \theta}{c_{\mathrm{f}}}\right)^{2}}=\left\{1-\left(\frac{c_{\mathrm{f} 2}}{c_{\mathrm{f}}}\right)^{2}+\left(\frac{c_{\mathrm{f} 2}}{c_{\mathrm{f}}} \cos \theta\right)^{2}\right\}^{\frac{1}{2}}
$$

Therefore, $R$ takes the following form:

$$
R=\frac{\rho_{2} c_{\mathrm{f} 2} \cos \theta-\rho c_{\mathrm{f}}\left\{1-\frac{c_{\mathrm{f} 2}^{2}}{c_{\mathrm{f}}^{2}}+\frac{c_{\mathrm{f} 2}^{2}}{c_{\mathrm{f}}^{2}} \cos ^{2} \theta\right\}^{\frac{1}{2}}}{\rho_{2} c_{\mathrm{f} 2} \cos \theta+\rho c_{\mathrm{f}}\left\{1-\frac{c_{\mathrm{f} 2}^{2}}{c_{\mathrm{f}}^{2}}+\frac{c_{\mathrm{f} 2}^{2}}{c_{\mathrm{f}}^{2}} \cos ^{2} \theta\right\}^{\frac{1}{2}}}
$$

In the above equation $\cos \theta$ can be obtained from the relation given below.

Dot product between the unit vectors $\hat{n}$ and $\hat{A}$ is given by

$$
\begin{aligned}
\hat{n} \cdot \hat{A} & =|\hat{n}||\hat{A}| \cos \theta=n_{1} a_{1}+n_{2} a_{2}+n_{3} a_{3} \\
& \Rightarrow \cos \theta=n_{3} a_{3}=a_{3}=\frac{z_{3}-y_{3}}{R_{2}}
\end{aligned}
$$

1.3.4.2 Pressure field computation in fluid 2 at point $Q$ Let us define two vectors $\underline{A}$ and $\underline{C}$, where $\underline{A}=\mathrm{CT}$ and $\underline{C}=\mathrm{TQ}$; then,

$$
\begin{aligned}
& \underline{A}=\left(z_{1}-y_{1}\right) \underline{e_{1}}+\left(z_{2}-y_{2}\right) \underline{e_{2}}+\left(z_{3}-y_{3}\right) \underline{e_{3}} \\
& \underline{C}=\left(x_{1}-z_{1}\right) \underline{e_{1}}+\left(x_{2}-z_{2}\right) \underline{e_{2}}+\left(x_{3}-z_{3}\right) \underline{e_{3}}
\end{aligned}
$$


Note that the magnitudes of vectors $\underline{A}$ and $\underline{C}$ are $R_{2}$ and $R_{3}$, respectively.

$$
\begin{aligned}
& R_{2}=\left\{\left(z_{1}-y_{1}\right)^{2}+\left(z_{2}-y_{2}\right)^{2}+\left(z_{3}-y_{3}\right)^{2}\right\}^{\frac{1}{2}} \\
& R_{3}=\left\{\left(x_{1}-z_{1}\right)^{2}+\left(x_{2}-z_{2}\right)^{2}+\left(x_{3}-z_{3}\right)^{2}\right\}^{\frac{1}{2}}
\end{aligned}
$$

Unit vectors $\hat{A}=\frac{\underline{A}}{R_{2}}$ and $\hat{C}=\frac{\underline{C}}{R_{3}}$

Unit vector $\hat{n}$ normal to the interface is given by

$\hat{n}=\left\{\begin{array}{l}0 \\ 0 \\ 1\end{array}\right\}$. Note that for an inclined interface, $\hat{n}=\left\{\begin{array}{l}n_{1} \\ n_{2} \\ n_{3}\end{array}\right\}$

From the problem geometry shown in Figure 1.27, the following equations are obtained:

$$
\begin{aligned}
& |\hat{n} \times \hat{A}|=\sin \theta \\
& \hat{n} \cdot \hat{A}=\cos \theta \\
& |\hat{n} \times \hat{C}|=\sin \theta_{2} \\
& \hat{n} \cdot \hat{C}=\cos \theta_{2}
\end{aligned}
$$

Let

$$
\hat{A}=\left\{\begin{array}{l}
a_{1} \\
a_{2} \\
a_{3}
\end{array}\right\}, \hat{C}=\left\{\begin{array}{l}
c_{1} \\
c_{2} \\
c_{3}
\end{array}\right\}
$$

From Eqs. (1.51) and (1.49)

$$
\begin{aligned}
& c_{3}=\cos \theta_{2}=\frac{x_{3}-z_{3}}{R_{3}} \\
& a_{3}=\cos \theta=\frac{z_{3}-y_{3}}{R_{2}}
\end{aligned}
$$

and

$$
\begin{aligned}
\hat{n} \times \hat{C} & =\operatorname{det}\left[\begin{array}{ccc}
e_{1} & e_{2} & e_{3} \\
n_{1} & n_{2} & n_{3} \\
c_{1} & c_{2} & c_{3}
\end{array}\right]=\left[\begin{array}{ccc}
0 & -n_{3} & n_{2} \\
n_{3} & 0 & -n_{1} \\
-n_{2} & n_{1} & 0
\end{array}\right]\left\{\begin{array}{l}
c_{1} \\
c_{2} \\
c_{3}
\end{array}\right\} \\
& =\left[\begin{array}{ccc}
0 & -1 & 0 \\
-1 & 0 & 0 \\
0 & 0 & 0
\end{array}\right]\left\{\begin{array}{l}
c_{1} \\
c_{2} \\
c_{3}
\end{array}\right\}=\left\{\begin{array}{c}
-c_{2} \\
c_{1} \\
0
\end{array}\right\} \\
& \Rightarrow|\hat{n} \times \hat{C}|^{2}=c_{1}^{2}+c_{2}^{2}=\sin ^{2} \theta_{2}
\end{aligned}
$$


or

$$
\sin ^{2} \theta_{2}=\frac{\left(x_{1}-z_{1}\right)^{2}+\left(x_{2}-z_{2}\right)^{2}}{R_{3}^{2}}
$$

Similarly,

$$
|\hat{n} \times \hat{A}|^{2}=a_{1}^{2}+a_{2}^{2}=\frac{\left(z_{1}-y_{1}\right)^{2}+\left(z_{2}-y_{2}\right)^{2}}{R_{2}^{2}}=\sin ^{2} \theta
$$

Because $\hat{A}, \hat{n}$, and $\hat{C}$ are located on the same plane

$$
\begin{aligned}
& \hat{n} \times \hat{A}=\hat{e}_{S} \sin \theta \\
& \hat{n} \times \hat{C}=\hat{e}_{S} \sin \theta_{2}
\end{aligned}
$$

where $\hat{e}_{\mathrm{S}}$ is the unit vector normal to the plane containing $\hat{A}, \hat{n}$, and $\hat{C}$.

From Eq. (1.56) and Snell's law (see Eq. (1.204) of Kundu(2004)) one can write

$$
\begin{aligned}
\frac{\hat{n} \times \hat{A}}{\sin \theta} & =\frac{\hat{n} \times \hat{C}}{\sin \theta_{2}} \\
& \Rightarrow \frac{\hat{n} \times \hat{A}}{c_{\mathrm{f}}}=\frac{\hat{n} \times \hat{C}}{c_{\mathrm{f} 2}}
\end{aligned}
$$

or

$$
\begin{aligned}
& \frac{1}{c_{\mathrm{f}}}\left\{\begin{array}{c}
-a_{2} \\
a_{1} \\
0
\end{array}\right\}=\frac{1}{c_{\mathrm{f} 2}}\left\{\begin{array}{c}
-c_{2} \\
c_{1} \\
0
\end{array}\right\} \\
& \Rightarrow\left\{\begin{array}{c}
-\frac{z_{1}-y_{1}}{c_{\mathrm{f}} R_{2}} \\
\frac{z_{2}-y_{2}}{c_{\mathrm{f}} R_{2}} \\
0
\end{array}\right\}=\left\{\begin{array}{c}
-\frac{x_{1}-z_{1}}{c_{\mathrm{f} 2} R_{3}} \\
\frac{x_{2}-z_{2}}{c_{\mathrm{f} 2} R_{3}} \\
0
\end{array}\right\}
\end{aligned}
$$

$z_{1}$ and $z_{2}$ can be obtained from Eq. (1.58) by minimizing the following error function:

$$
E=\left(\frac{z_{1}-y_{1}}{c_{\mathrm{f}} R_{2}}+\frac{z_{1}-x_{1}}{c_{\mathrm{f} 2} R_{3}}\right)^{2}+\left(\frac{z_{2}-y_{2}}{c_{\mathrm{f}} R_{2}}+\frac{z_{2}-x_{2}}{c_{\mathrm{f} 2} R_{3}}\right)^{2}
$$

$E$ can be minimized by some optimization technique such as simplex algorithm. In MATLAB code, "fminsearch" function can be used for this purpose. After evaluating 
$z_{1}$ and $z_{2}$, the pressure at point Q can be obtained from the following equation:

$$
p\left(x_{1}, x_{2}, x_{3}\right)=-\frac{i \omega \rho_{\mathrm{f}} v_{o}}{2 \pi} \int_{\mathrm{S}} \frac{\left.T_{p} \exp \left\lfloor i k_{\mathrm{f}} R_{2}+k_{\mathrm{f} 2} R_{3}\right)\right\rfloor}{\sqrt{R_{2}+\frac{c_{\mathrm{f} 2}}{c_{\mathrm{f}}} R_{3}} \sqrt{R_{2}+R_{3} \frac{c_{\mathrm{f} 2} \cos ^{2} \theta}{c_{\mathrm{f}} \cos ^{2} \theta_{2}}}} d S
$$

The numerator of the above integrand is similar to that in Eq. (1.16), the only difference is that in Eq. (1.60) the numerator has been multiplied by $T_{p}$, the transmission coefficient at the interface, and the argument of the exponential term has two entries, corresponding to the ray paths CT and TQ. However, the denominator of Eq. (1.60) is much more complex in comparison to the one given in Eq. (1.16). Derivation of this denominator expression can be found in Schmerr (1998).

The transmission coefficient is given in Eq. (1.208) of Kundu(2004).

$$
T_{p}=\frac{2 \rho_{\mathrm{f} 2} c_{\mathrm{f} 2} \cos \theta_{2}}{\rho_{\mathrm{f} 2} c_{\mathrm{f} 2} \cos \theta_{1}+\rho_{\mathrm{f}} c_{\mathrm{f}} \cos \theta_{2}}
$$

As mentioned above, in this case, the incident angle is $\theta$, angle of transmission is $\theta_{2}$, fluid densities are $\rho_{\mathrm{f}}$ and $\rho_{\mathrm{f} 2}$, and acoustic wave speeds in the two fluids are $c_{\mathrm{f}}$ and $c_{\mathrm{f} 2}$. Angle $\theta_{2}$ can be expressed in terms of the incident angle $\theta$ (see Eq. (1.46)); then, $T_{p}$ takes the following form,

$$
T_{p}=\frac{2 \rho_{\mathrm{f} 2} c_{\mathrm{f} 2} \cos \theta}{\rho_{\mathrm{f} 2} c_{\mathrm{f} 2} \cos \theta+\rho_{\mathrm{f}} c_{\mathrm{f}}\left\{1-\frac{c_{\mathrm{f} 2}^{2}}{c_{\mathrm{f}}^{2}}+\frac{c_{\mathrm{f} 2}^{2}}{c_{\mathrm{f}}^{2}} \cos ^{2} \theta\right\}^{\frac{1}{2}}}
$$

\subsubsection{DPSM technique for ultrasonic field modeling in nonhomogeneous fluid}

The steps discussed in Section 1.2.4 are based on the Rayleigh-Sommerfield integral representation for the pressure field computation in fluids 1 and 2. In this section, an alternative technique based on DPSM, developed by Placko and Kundu (2001) for ultrasonic problems, is generalized to include the nonhomogeneous fluid case. In the DPSM technique, the interface is replaced by a layer of equivalent point sources instead of tracing the rays from the transducer face to the point of interest in fluid media 1 or 2, as done in Section 1.3.4.

1.3.5.1 Field computation in fluid 1 The field in fluid 1 is computed by superimposing the contributions of the two layers of point sources distributed over the transducer face and the interface, respectively, as shown in Figure 1.28. The two layers of the sources are located at a small distance $r_{\mathrm{S}}$ away from the transducer face and interface, respectively, such that the apex of the spheres (of radius $r_{\mathrm{S}}$ ) touch the transducer face or interface, as shown in the figure. 


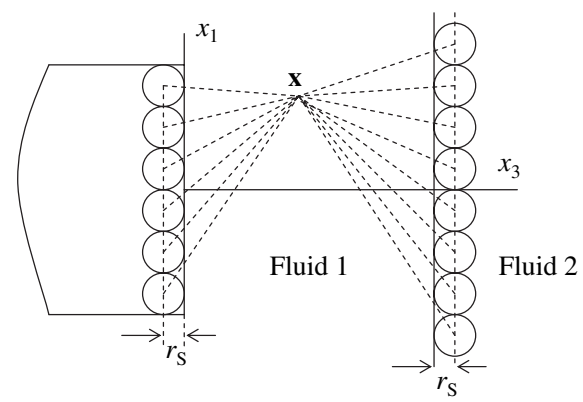

Figure 1.28 Point sources (at the center of small circles) for computing ultrasonic field in fluid 1

The strength of the point sources distributed along the transducer surface can be obtained from Eq. (1.25g) or (1.25i). For finding the strength of the point sources attached to the interface, velocity components at the interface due to the reflected waves at the interface are to be matched, as described below.

As shown in Figure 1.29, any point $P$ in fluid 1 can receive only two rays, 1 and 2 , from a single point source on the transducer surface. Ray 1 is the direct ray reaching $P$, and ray 2 arrives at $P$ after being reflected at the interface. The total ultrasonic field at point $\mathrm{P}$ can be obtained by superimposing the contributions of a number of point sources $\left(\mathbf{y}_{m}\right)$ distributed over the transducer surface. The total field at $\mathrm{P}$, generated only by the reflected rays (ray number 2), from all the point sources on the transducer surface should be the same as the total contributions of all point sources distributed over the interface.

Let us take a point $\mathrm{P}$ at $\mathbf{x}_{n}$ on the interface. In Figure 1.29, the point $\mathrm{P}$ is shown very close to the interface. Let us assume that this point is now moved to the interface. Let there be $N$ point sources $\left(\mathbf{y}_{m}, m=1,2, \ldots, N\right)$ on the transducer surface and $M$ points $\left(\mathbf{x}_{n}, n=1,2, \ldots, M\right)$ on the interface where the boundary conditions should be satisfied. If the boundary conditions are to be satisfied for the three components of velocity at all $M$ points, then there are a total of $3 M$ boundary conditions. It should be noted here that for nonviscous fluids, matching of the normal velocity component only is sufficient. In that case, from $M$ points $M$ boundary conditions will be obtained.

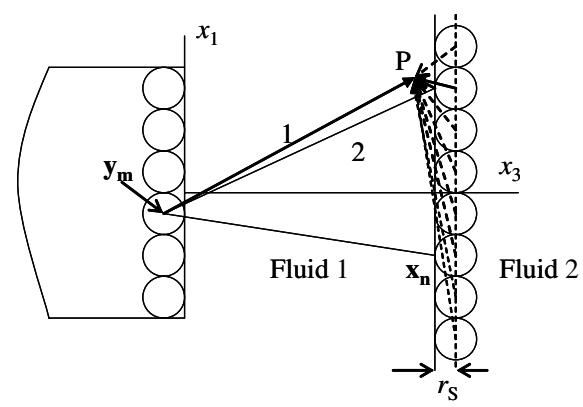

Figure 1.29 Point $\mathrm{P}$ can receive two rays, 1 (direct ray) and 2 (reflected from the interface), from a single point source $\mathbf{y}_{\mathbf{m}}$. 
Velocity components at $M$ interface points due to ray 1 only (ignoring reflection) can be easily obtained from the $N$ triplet or elementary simple point sources in the following manner (see Eq. (1.25j)):

$$
\mathbf{V}_{\mathrm{T}}^{i}=\mathbf{M}_{\mathrm{TS}}^{i} \mathbf{A}_{\mathrm{S}}
$$

where $\mathbf{V}_{\mathrm{T}}^{i}$ is the $(3 M \times 1)$ or $(M \times 1)$ vector of the velocity components at the target points $\left(\mathbf{x}_{\mathrm{n}}\right)$ on the interface due to the incident beam only. $\mathbf{A}_{S}$ is the $(3 N \times 1)$ or $(N \times 1)$ vector of the point source strengths on the transducer surface. For the triplet source, there are three point sources inside every small sphere. The following formulation is given for triplet source only but can be easily modified to simple elemental sources used for nonviscous fluids.

Because the normal velocity component $\left(v_{0}\right)$ at the transducer surface is known, $\mathbf{A}_{\mathbf{S}}$ can be obtained from Eq. (1.25g) or (1.25i). $\mathbf{M}_{\mathrm{TS}}^{i}$ is the $(3 M \times 3 N)$ matrix that relates the two vectors $\mathbf{V}_{\mathrm{T}}^{i}$ and $\mathbf{A}_{\mathrm{S}}$ of Eq. (1.62). Note that the components of $\mathbf{M}_{\mathrm{TS}}^{i}$ are identical to those for $\mathbf{M}_{\mathrm{TS}}$ given in Eq. (1.25k).

In Eq. (1.62) and in subsequent equations, the superscripts and subscripts have the following meanings:

\section{Superscripts}

$\mathrm{i}$ - direct incident ray

$\mathrm{r}-$ reflected ray

$\mathrm{t}-$ transmitted ray

\section{Subscripts}

$\mathrm{S}-$ ultrasonic source or transducer points

I - interface points

$\mathrm{T}$ - target points or observation points (these points can be placed anywhere-in fluid 1 , fluid 2, on the transducer surface, or on the interface).

For the reflected field computation at the interface, the velocity vector and the source strength vector are computed in a similar manner.

$$
\mathbf{V}_{\mathrm{T}}^{\mathrm{r}}=\mathbf{M}_{\mathrm{TS}}^{\mathrm{r}} \mathbf{A}_{\mathrm{S}}
$$

where $\mathbf{V}_{\mathrm{T}}^{\mathrm{r}}$ is the $(3 M \times 1)$ vector of the three velocity components at the target points $\left(\mathbf{x}_{n}\right)$ on the interface due to the reflected beam only (ray 2 of Fig. 1.29). $\mathbf{M}_{\mathrm{TS}}^{\mathrm{r}}$ is the $(3 M \times 3 N)$ matrix that relates the two vectors $\mathbf{V}_{\mathrm{T}}^{\mathrm{r}}$ and $\mathbf{A}_{\mathrm{S}}$ of Eq. (1.63). Note that components of $\mathbf{M}_{\mathrm{TS}}^{\mathrm{r}}$ can be obtained by multiplying $\mathbf{M}_{\mathrm{TS}}^{\mathrm{i}}$ by appropriate reflection coefficients for velocity fields.

Next we would like to obtain the same $\mathbf{V}_{\mathrm{T}}^{\mathrm{r}}$ vector from the $3 M$ point sources distributed along the interface. Within each sphere shown in Figure 1.29, three point 
sources or triplet (see Fig. 1.25) are placed; thus, from $M$ spheres one gets $3 M$ sources. Note that the interface point sources are located around the centers of the small spheres of Figure 1.29, and three sources of each triplet are placed parallel to the interface. Points $\mathbf{x}_{n}$ are located on the surface of the small spheres. $\mathbf{V}_{\mathrm{T}}^{\mathrm{r}}$ at $M$ points, generated by the $3 M$ sources at the interface, can be written as

$$
\mathbf{V}_{\mathrm{T}}^{\mathrm{r}}=\mathbf{M}_{\mathrm{TI}}^{\mathrm{i}} \mathbf{A}_{\mathrm{I}}
$$

where $\mathbf{V}_{\mathrm{T}}^{\mathrm{r}}$ is the $(3 M \times 1)$ vector, same as in Eq. (1.63). $\mathbf{A}_{\mathrm{I}}$ is the $(3 M \times 1)$ vector of the strength of interface sources; this vector is unknown. $\mathbf{M}_{\mathrm{TI}}^{\mathrm{i}}$ is the $(3 M \times 3 M)$ matrix that relates the two vectors $\mathbf{V}_{\mathrm{T}}^{\mathrm{r}}$ and $\mathbf{A}_{\mathrm{I}}$ of Eq. (1.64). Note that the components of $\mathbf{M}_{\mathrm{TI}}^{\mathrm{i}}$ are similar to those for $\mathbf{M}_{\mathrm{SS}}$ given in Eq. (1.25e) and $\mathbf{M}_{\mathrm{TS}}$ of Eq. (1.25k). The two variables $x_{j m}^{n}$ and $r_{m}^{n}$ for $\mathbf{M}_{\mathrm{TI}}^{\mathrm{i}}$ computation can be obtained after knowing the point source coordinates $\mathbf{z}_{m}(m=1,2, \ldots, 3 M)$ distributed along the interface, and coordinates $\mathbf{x}_{n}(n=1,2, \ldots, M)$ of the interface points.

From Eqs. (1.63) and (1.64)

$$
\mathbf{A}_{\mathrm{I}}=\left[\mathbf{M}_{\mathrm{TI}}^{\mathrm{i}}\right]^{-1} \mathbf{V}_{\mathrm{T}}^{\mathrm{r}}=\left(\left[\mathbf{M}_{\mathrm{TI}}^{\mathrm{i}}\right]^{-1} \mathbf{M}_{\mathrm{TS}}^{\mathrm{r}}\right) \mathbf{A}_{\mathrm{S}}
$$

Eq. (1.65) gives the strength of the interface sources. After obtaining the interface source strengths, the ultrasonic field at any set of target points $\mathbf{x}_{n}\left(n=1,2, \ldots, N_{\mathrm{T}}\right)$ between the transducer face and interface can be obtained by adding the contributions of the incident waves from the two layers of point sources at the transducer face and interface as shown in Figure 1.28. In other words, the field at any point can be obtained by adding the expressions given in Eqs. (1.62) and (1.64).

$$
\mathbf{V}_{\mathrm{T}}=\mathbf{V}_{\mathrm{T}}^{\mathrm{i}}+\mathbf{V}_{\mathrm{T}}^{\mathrm{r}}=\mathbf{M}_{\mathrm{TS}}^{\mathrm{i}} \mathbf{A}_{\mathrm{S}}+\mathbf{M}_{\mathrm{TI}}^{\mathrm{i}} \mathbf{A}_{\mathrm{I}}
$$

The only difference between the two components of Eq. (1.66) and those in Eqs. (1.62) and (1.64) is in the definitions of $x_{j m}^{n}$ and $r_{m}^{n}$ for $\mathbf{M}_{\mathrm{TI}}^{\mathrm{i}}$ and $\mathbf{M}_{\mathrm{TS}}^{\mathrm{i}}$. In Eqs. (1.62) and (1.64), the target points are located on the interface, whereas in Eq. (1.66) the target points are in between the transducer face and the interface. Therefore, the values of $x_{j m}^{n}$ and $r_{m}^{n}$ will change accordingly. As mentioned earlier $r_{m}^{n}$ is the distance between the $m$ th point source and $n$th target point, $x_{j m}^{n}$ are the three components of $r_{m}^{n}$.

1.3.5.1.1 Approximations in computing the field The approximation of the above section in deriving Eq. (1.66) is that the presence of the interface does not affect the source strength vector $\mathbf{A}_{\mathrm{S}}$. Note that $\mathbf{A}_{\mathrm{S}}$ of Eq. (1.62) is computed from Eq. (1.25g) or (1.25i). With this assumption, the velocity vector computed on the transducer surface, using Eq. (1.66), will give a different value than $v_{0}$. If the interface is close to the transducer surface, then the transducer surface velocity will be significantly different from $v_{0}$ due to the interface effect.

To make sure that the velocity vector on the interface is equal to a constant value $\left(v_{0}\right)$ in the $x_{3}$ direction and zero in $x_{1}$ and $x_{2}$ directions, the following formulation is followed. 
Similar to the previous section, it is again assumed that there are $N$ triplet sources on the transducer surface and $M$ sources along the interface. The velocity vector on the transducer surface, due to the point sources representing the transducer effect only, can be obtained from Eq. (2.49).

$$
\mathbf{V}_{\mathrm{S}}^{\mathrm{i}}=\mathbf{M}_{\mathrm{SS}}^{\mathrm{i}} \mathbf{A}_{\mathrm{S}}
$$

where $\mathbf{V}_{\mathrm{S}}^{\mathrm{i}}$ is the $(3 N \times 1)$ vector of the velocity components at the transducer surface. $\mathbf{A}_{\mathrm{S}}$ is the $(3 N \times 1)$ vector of the point source strengths distributed over the transducer face, and $\mathbf{M}_{\mathrm{SS}}^{\mathrm{i}}$ is the $(3 N \times 3 N)$ matrix, identical to the one given in Eq. (1.25e).

In the same manner, velocity components on the transducer surface due to the interface sources are given by,

$$
\mathbf{V}_{\mathrm{S}}^{\mathrm{r}}=\mathbf{M}_{\mathrm{SI}}^{\mathrm{i}} \mathbf{A}_{\mathrm{I}}
$$

The above equation is obtained from Eq. (1.64), when the target points are placed on the transducer surface. Here, $\mathbf{V}_{\mathrm{S}}^{\mathrm{r}}$ is a $(3 N \times 1)$ vector of the velocity components at $N$ points on the transducer surface, $\mathbf{A}_{\mathrm{I}}$ is the $(3 M \times 1)$ vector of the interface source strengths, and $\mathbf{M}_{\mathrm{SI}}^{\mathrm{i}}$ is the $(3 N \times 3 M)$ matrix, similar to the one given in Eq. $(1.25 \mathrm{k})$.

Adding Eqs. (1.66a) and (1.66b) the total velocity at the transducer surface is obtained.

$$
\mathbf{V}_{\mathrm{S}}=\mathbf{V}_{\mathrm{S}}^{\mathrm{i}}+\mathbf{V}_{\mathrm{S}}^{\mathrm{r}}=\mathbf{M}_{\mathrm{SS}}^{\mathrm{i}} \mathbf{A}_{\mathrm{S}}+\mathbf{M}_{\mathrm{SI}}^{\mathrm{i}} \mathbf{A}_{\mathrm{I}}
$$

Substituting Eq. (1.65) into Eq. (1.66c):

$$
\begin{aligned}
\mathbf{V}_{\mathrm{S}} & =\mathbf{M}_{\mathrm{SS}}^{\mathrm{i}} \mathbf{A}_{\mathrm{S}}+\mathbf{M}_{\mathrm{SI}}^{\mathrm{i}} \mathbf{A}_{\mathrm{I}}=\mathbf{M}_{\mathrm{SS}}^{\mathrm{i}} \mathbf{A}_{\mathrm{S}}+\mathbf{M}_{\mathrm{SI}}^{\mathrm{i}}\left[\mathbf{M}_{\mathrm{TI}}^{\mathrm{i}}\right]^{-1} \mathbf{M}_{\mathrm{TS}}^{\mathrm{r}} \mathbf{A}_{\mathrm{S}} \\
& \Rightarrow \mathbf{V}_{\mathrm{S}}=\left[\mathbf{M}_{\mathrm{SS}}^{\mathrm{i}}+\mathbf{M}_{\mathrm{SI}}^{\mathrm{i}}\left[\mathbf{M}_{\mathrm{TI}}^{\mathrm{i}}\right]^{-1} \mathbf{M}_{\mathrm{TS}}^{\mathrm{r}}\right] \mathbf{A}_{\mathrm{S}}
\end{aligned}
$$

or

$$
\mathbf{A}_{\mathrm{S}}=\left[\mathbf{M}_{\mathrm{SS}}^{\mathrm{i}}+\mathbf{M}_{\mathrm{SI}}^{\mathrm{i}}\left[\mathbf{M}_{\mathrm{TI}}^{\mathrm{i}}\right]^{-1} \mathbf{M}_{\mathrm{TS}}^{\mathrm{r}}\right]^{-1} \mathbf{V}_{\mathrm{S}}
$$

where

$$
\mathbf{V}_{\mathrm{S}}=\left[\begin{array}{llllllllll}
0 & 0 & v_{0} & 0 & 0 & v_{0} & \ldots \ldots \ldots \ldots & 0 & 0 & v_{0}
\end{array}\right]^{\mathrm{T}}
$$

If $\mathbf{A}_{\mathrm{S}}$ is computed from Eq. (1.66e) instead of Eq. (1.25g), then the constant velocity at the transducer surface is guaranteed even when the interface is located very close to the transducer surface.

1.3.5.2 Field in fluid 2 For ultrasonic field computation in fluid 2, only one layer of point sources, adjacent to the interface, is considered as shown in Figure 1.30. The total field at $\mathbf{x}$ should be the superposition of fields generated by all these point sources, located at various distances from $\mathbf{x}$, as shown by the dotted lines in Figure 1.30. Strengths of these sources are obtained, as before, by equating the velocity components computed by the point sources, distributed along the interface, to those obtained from the transmitted wave contribution. 


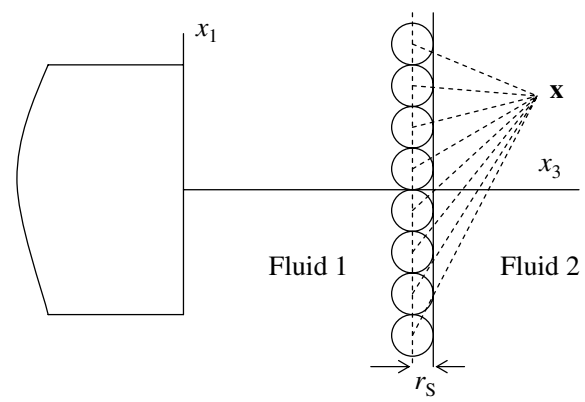

Figure 1.30 One layer of point sources (at the center of small circles) for computing ultrasonic field in fluid 2

Following similar analysis as outlined in section 2.5.1, strengths of the interface point sources in this case can be obtained from the relation (see Eq. (1.65)).

$$
\mathbf{A}_{\mathrm{I}}=\left[\mathbf{M}_{\mathrm{TI}}^{\mathrm{i}}\right]^{-1} \mathbf{V}_{\mathrm{T}}^{\mathrm{t}}=\left(\left[\mathbf{M}_{\mathrm{TI}}^{\mathrm{i}}\right]^{-1} \mathbf{M}_{\mathrm{TS}}^{\mathrm{t}}\right) \mathbf{A}_{\mathrm{S}}
$$

where $\mathbf{A}_{\mathrm{I}}$ is the $(3 M \times 1)$ vector of the interface source strengths, $\mathbf{M}_{\mathrm{TI}}^{\mathrm{i}}$ is the $(3 M \times 3 M)$ matrix that relates the two vectors $\mathbf{V}_{\mathrm{T}}^{\mathrm{t}}$ and $\mathbf{A}_{\mathrm{I}}$, and $\mathbf{M}_{\mathrm{TS}}^{\mathrm{t}}$ is the $(3 M \times 3 N)$ matrix relating the velocity vector $\mathbf{V}_{\mathrm{T}}^{\mathrm{t}}$ at the interface points and $\mathbf{A}_{\mathrm{S}}$, the source strength vector for point sources, distributed along the transducer face.

Note that in this case, the equations relating the interface velocity components to the transducer source strengths and interface source strengths are similar to Eqs. (1.63) and (1.64) and can be written as

$$
\begin{aligned}
\mathbf{V}_{\mathrm{T}}^{\mathrm{t}} & =\mathbf{M}_{\mathrm{TS}}^{\mathrm{t}} \mathbf{A}_{\mathrm{S}} \\
\mathbf{V}_{\mathrm{T}}^{\mathrm{t}} & =\mathbf{M}_{\mathrm{TI}}^{\mathrm{i}} \mathbf{A}_{\mathrm{I}}
\end{aligned}
$$

After computing the interface source strengths using Eq. (1.67), the ultrasonic field at any new target points $\mathbf{x}_{n}\left(n=1,2, \ldots, N_{T}\right)$ on the right side of the interface (or in fluid 2) can be obtained from Eq. (1.69). While computing the field at new points, appropriate changes in the values of $x_{j m}^{n}$ and $r_{m}^{n}$ appearing in matrix $\mathbf{M}_{\mathrm{TI}}^{\mathrm{i}}$ should be taken into account. As mentioned earlier, $r_{m}^{n}$ is the distance between the $m$ th point source and $n$th target point, $x_{j m}^{n}$ are the three components of $r_{m}^{n}$.

\subsubsection{Ultrasonic field in the presence of a scatterer}

DPSM technique is then applied to model ultrasonic field near a scatterer of finite dimensions for which no closed-form analytical solution exists. Problem geometry showing the transducer and scatterer is given in Figure 1.31.

To compute the ultrasonic field in front of a scatterer (left of the scatterer), point sources are distributed along the transducer face and the solid-fluid interface as well as along the imaginary interface (extending the front face of the solid scatterer, shown by the dotted line in Figure 1.31). Triplet sources are located around the centres of the 


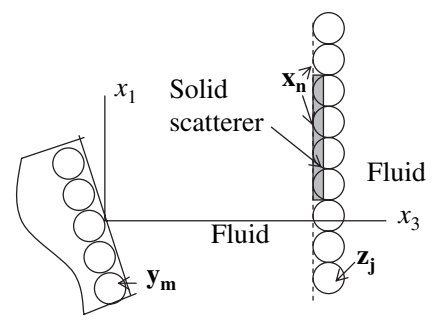

Figure 1.31 A finite solid scatterer immersed in a fluid in front of a transducer face - two layers of point source contribute to the ultrasonic field in between the transducer and the scatterer.

small spheres. Strength of the point sources on the transducer face is known from the normal velocity component $v_{0}$ of the transducer surface (Eq. (1.25i) or (1.66e)). However, strength of the point sources distributed along the real and imaginary interface is not known. This is carried out in a manner similar to the one described in Section 2.5.1. The only difference here is that in Eq. (1.63), $\mathbf{M}_{\mathrm{TS}}^{\mathrm{r}}$ must be obtained by multiplying $\mathbf{M}_{\mathrm{TS}}^{\mathrm{i}}$ by appropriate reflection coefficients. The technique to compute the reflection coefficient for this case differs from the one given in Section 2.5.1. In the previous case, the same expression of the reflection coefficient (Eq. (1.47)) was used for all interface points $\mathbf{x}_{n}$. However, for this problem geometry when the interface points $\mathbf{x}_{n}$ are located on the scatterer surface, then the reflection coefficient for a solid plate immersed in a fluid (see Section 1.2.17 of Kundu(2004)) should be used. However, when the interface points $\mathbf{x}_{n}$ are located on the dotted line, along the imaginary interface between two identical fluids, then the reflection coefficient should be zero. Except for this difference in the reflection coefficient definition, the steps to compute the interface source strengths for these two problem geometries are identical, and the source strength vector can be obtained from Eq. (1.65)

$$
\mathbf{A}_{\mathrm{I}}=\left[\mathbf{M}_{\mathrm{TI}}^{\mathrm{i}}\right]^{-1} \mathbf{V}_{\mathrm{T}}^{\mathrm{r}}=\left(\left[\mathbf{M}_{\mathrm{TI}}^{\mathrm{i}}\right]^{-1} \mathbf{M}_{\mathrm{TS}}^{\mathrm{r}}\right) \mathbf{A}_{\mathrm{S}}
$$

For computing the ultrasonic field behind the scatterer, or on the right side of the dotted line, the point sources should be taken as shown in Figure 1.32. Note that now some of the point sources are aligned with the right edge of the scatterer whereas the

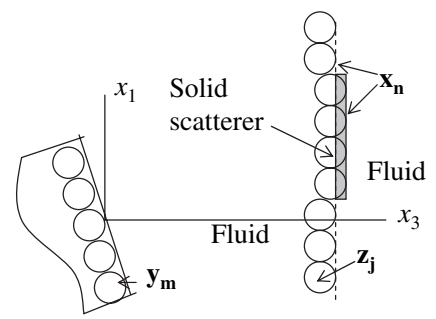

Figure 1.32 A finite solid scatterer immersed in a fluid in front of a transducer face - only the right layer of point sources contribute to the ultrasonic field in the fluid on the right side of the scatterer. 
rest are aligned with the imaginary interface along the left edge, and marked by the dotted line. Of course, for thin scatterer these two planes coincide.

Following similar steps as in Section 2.5.2, the strengths of the interface point sources in this case can be obtained from Eq. (1.67),

$$
\mathbf{A}_{\mathrm{I}}=\left[\mathbf{M}_{\mathrm{TI}}^{\mathrm{i}}\right]^{-1} \mathbf{V}_{\mathrm{T}}^{\mathrm{t}}=\left(\left[\mathbf{M}_{\mathrm{TI}}^{\mathrm{i}}\right]^{-1} \mathbf{M}_{\mathrm{TS}}^{\mathrm{t}}\right) \mathbf{A}_{\mathrm{S}}
$$

where $\mathbf{A}_{\mathrm{I}}$ is the $(3 M \times 1)$ vector of the interface source strengths, $\mathbf{M}_{\mathrm{TI}}^{\mathrm{i}}$ is the $(3 M \times$ $3 M)$ matrix that relates the velocity vector $\mathbf{V}_{\mathrm{T}}^{\mathrm{t}}$ at the interface points $\mathbf{x}_{n}$ to the interface source strengths $\mathbf{A}_{\mathrm{I}}$, and $\mathbf{M}_{\mathrm{TS}}^{\mathrm{t}}$ is the $(3 M \times 3 N)$ matrix that relates the velocity vector $\mathbf{V}_{\mathrm{T}}^{\mathrm{t}}$ at the interface points to the transducer source strength vector $\mathbf{A}_{\mathrm{S}}$.

Note that in this case, equations relating the interface velocity components to the transducer source strengths and interface source strengths are similar to Eqs. (1.63) and (1.64) and can be written as

$$
\begin{aligned}
\mathbf{V}_{\mathrm{T}}^{\mathrm{t}} & =\mathbf{M}_{\mathrm{TS}}^{\mathrm{t}} \mathbf{A}_{\mathrm{S}} \\
\mathbf{V}_{\mathrm{T}}^{\mathrm{t}} & =\mathbf{M}_{\mathrm{TI}}^{\mathrm{i}} \mathbf{A}_{\mathrm{I}}
\end{aligned}
$$

After computing the interface source strengths using Eq. (2.54), the ultrasonic field at any new target points $\mathbf{x}_{n}\left(n=1,2, \ldots, N_{T}\right)$ on the right side of the interface can be obtained from Eq. (1.69). For computing the field at new points, appropriate changes in the values of $x_{j m}^{n}$ and $r_{m}^{n}$ appearing in matrix $\mathbf{M}_{\mathrm{TI}}^{\mathrm{i}}$ should be taken into account. As mentioned earlier, $r_{m}^{n}$ is the distance between the $m$ th point source and $n$th target point and $x_{j m}^{n}$ are the three components of $r_{m}^{n}$.

\subsubsection{Numerical results}

Sections 1.3.1-1.3.6 describe the theory of the ultrasonic field modeling by using the DPSM technique in homogeneous and nonhomogeneous fluids. Based on this theory the authors have developed a number of MATLAB computer codes to model the ultrasonic fields generated by the ultrasonic transducers of finite dimension, which are immersed in a fluid. In the simplest case, the transducer is immersed in a homogeneous fluid. More complex problem geometries involve two fluids with a plane interface and a solid scatterer of finite size immersed in a homogeneous fluid. The numerical results clearly show how the ultrasonic field decays as the distance from the transducer increases and the field becomes more collimated as the size of the transducer increases. It also shows that the field is reflected and transmitted at an interface, and how a finite size scatterer can give rise to the reflection and transmission as well as diffraction of the incident field.

1.3.7.1 Ultrasonic field in a homogeneous fluid In this example the ultrasonic field in front of a flat circular, flat rectangular, and concave circular transducer faces are generated. The transducer front face geometries are shown in Figure 1.33. The area of the flat transducer face is $5.76 \mathrm{~mm}^{2}$ for both circular and square transducers. Note that a $2.7 \mathrm{~mm}$ diameter circular transducer gives an area of $5.76 \mathrm{~mm}^{2}$. A concave 


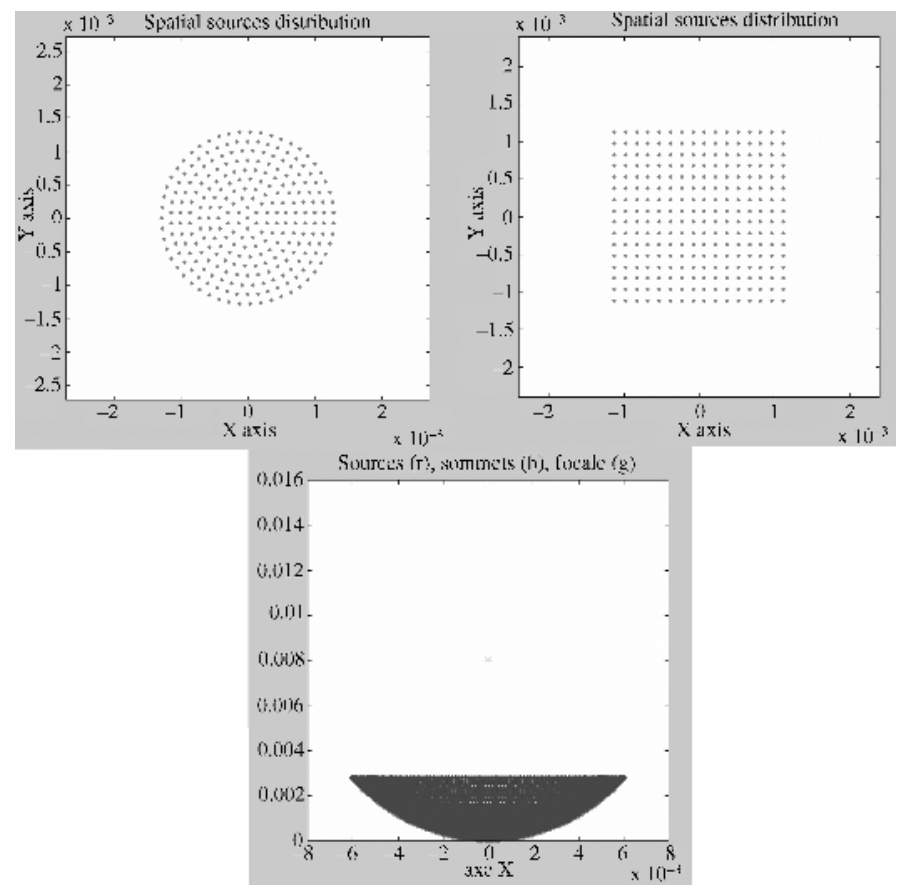

Figure 1.33 Distribution of the point sources just behind the front face (see Fig. 1.24) of a flat circular (top left) and flat square (top right) transducer. Bottom figure - side view of the concave front face of a transducer

transducer face has different dimensions; its diameter is $12.7 \mathrm{~mm}(0.5 \mathrm{in}$.$) and its$ radius of curvature is $8 \mathrm{~mm}$, as shown in Figure 1.33. All of the dimensions in the figure are given in meter, but the scales are not necessarily the same in the horizontal and vertical directions. These three transducers are denoted as circular, square, and focused transducers. Note that the flat transducer face is located on the $x y$ plane. We would like to compute the ultrasonic field in front of the transducer face in the $x z$ plane or the $y z$ plane. Both $x z$ and $y z$ planes are planes of symmetry and are perpendicular to each other.

The ultrasonic pressure field variations along the $x z$ and $y z$ planes in front of the transducer face are shown in Figure 1.34 for $5 \mathrm{MHz}$ frequency of the transducers. The top-left and top-right images of Figure 1.34 are for the circular and square transducers, respectively. Note that the field is less collimated for the square transducer. For both transducer geometries the ultrasonic field has a number of peaks (or maxima) and dips (or minima) along the central axis ( $z$-axis) of the transducer near the transducer face. The peaks and dips are a result of constructive and destructive interferences between the fields generated by different point sources on the transducer face.

For the concave transducer the field intensity increases as we approach the focal point. Note that the focal point is at a distance of $8 \mathrm{~mm}$ from the transducer face, whereas the plot is shown for a distance varying from 3 to $6 \mathrm{~mm}$.

It should be mentioned here that the focused transducer surface area is 21 times that of the flat transducers. To maintain the same spacing between neighboring point 


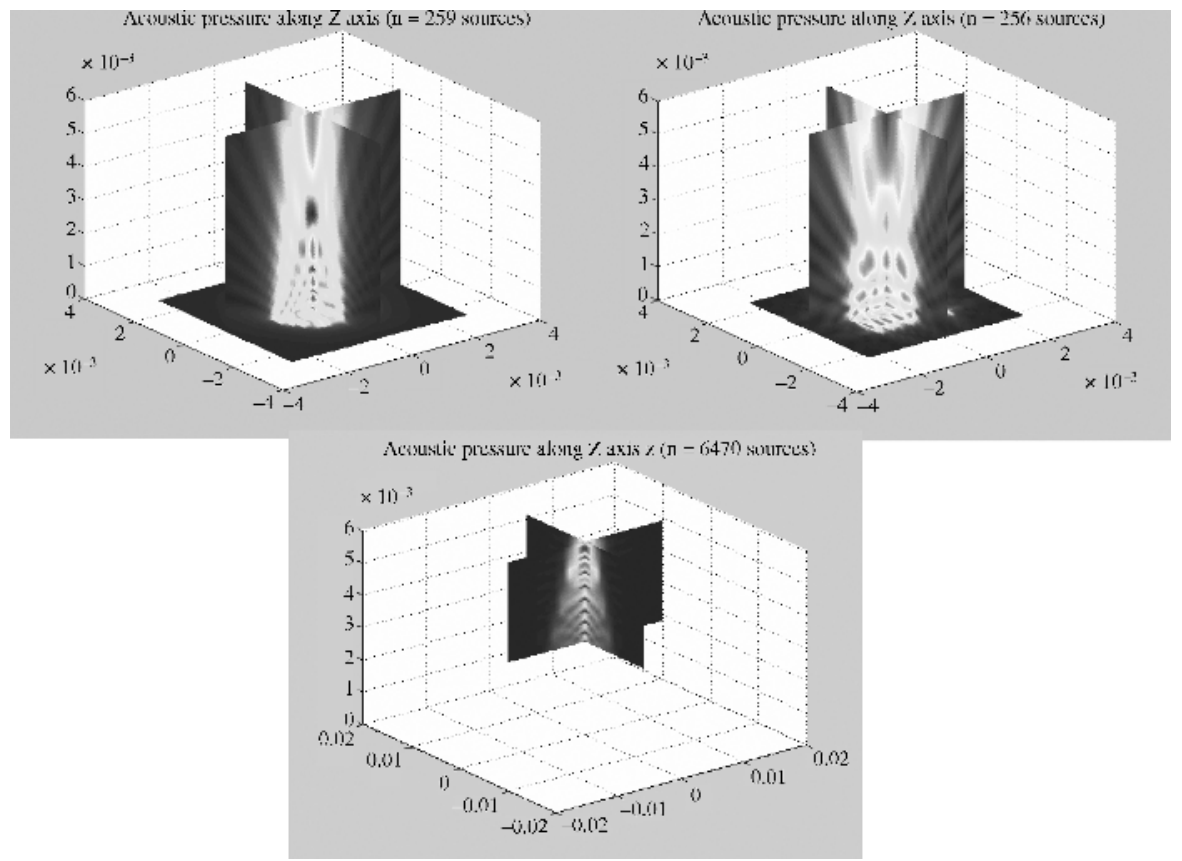

Figure 1.34 Ultrasonic pressure fields generated by a circular (top left), a square (top right), and a concave circular (bottom) transducer. Transducer face geometries are shown in Fig.1.33. Transducer frequency is $1 \mathrm{MHz}$. The surface area of the flat transducers is $5 \mathrm{~mm}^{2}$. The concave transducer has a radius of curvature of $8 \mathrm{~mm}$, and the diameter of its periphery is $12.7 \mathrm{~mm}$. The number of point sources is 259 for the top-left figure, 256 for the top-right figure, and 6470 for the bottom figure. The ultrasonic field is plotted up to an axial distance of $6 \mathrm{~mm}$. Note that the focal point for the concave transducer is at a distance of $8 \mathrm{~mm}$, which is beyond the plotted region.

sources (see Section 1.3.2.3), the number of point sources for the focused transducer is made about 25 times that of the flat transducers. Thus, the number of point sources for the focused transducer is 6470 whereas for the flat transducers the two numbers are 256 and 259 , respectively.

Variations of the pressure field along the z-axis, in front of the transducer face, are clearly shown in Figure 1.35. The top two figures of 1.35 are for the circular and square transducers, and the bottom figure is for the focused transducer. The analytical solutions (Eq. (1.16b) for the flat circular transducer and Eq. (1.32) for the focused transducer) give results that are very close to the one obtained by the DPSM technique (Eq. (1.25j)), see Figure 1.35. Three peaks between 0 and $4 \mathrm{~mm}$ along the $z$-axis in Figure 1.35 correspond for both circular and square transducers to the three bright red dots in Figure 1.34 along the central axis of the ultrasonic beam.

\section{Example 1.3.4}

Check if Eq. (1.30) is satisfied for the flat circular cylinder with 259 point sources for $1 \mathrm{MHz}$ signal. 


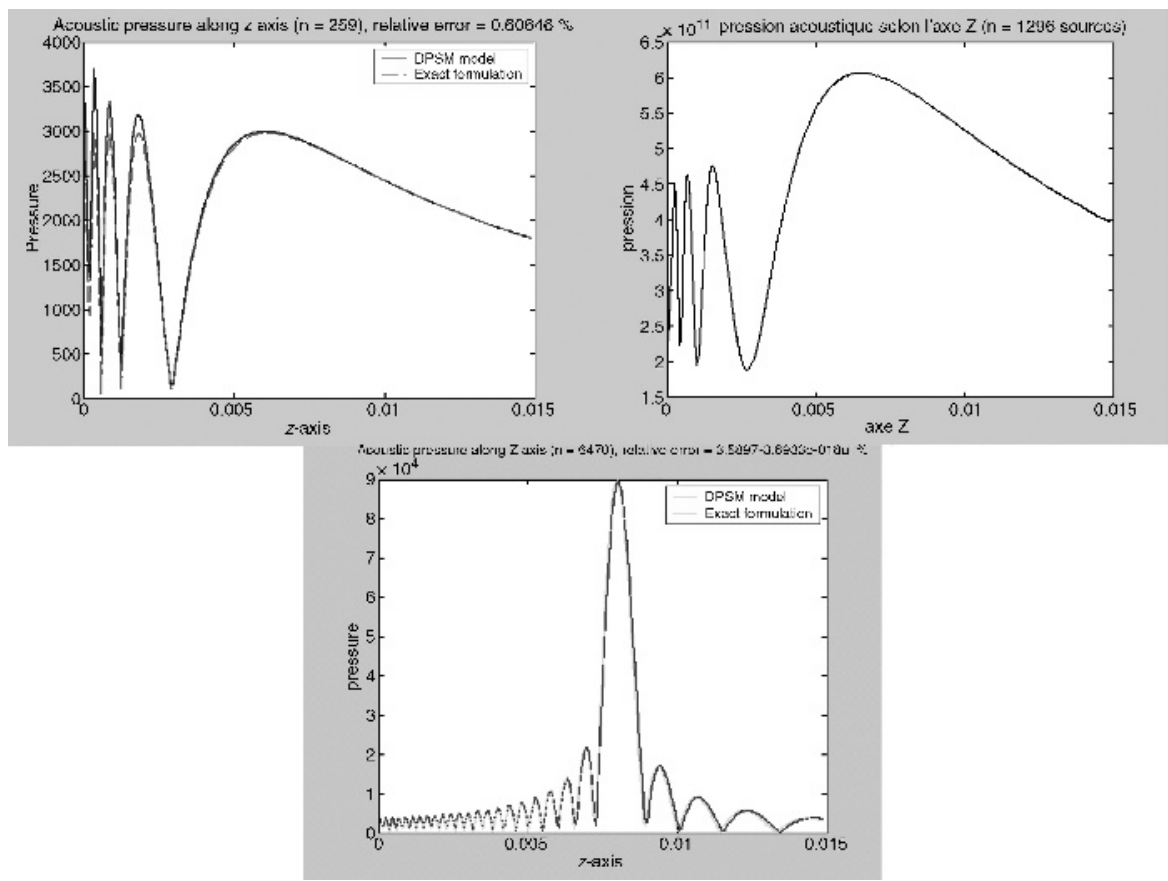

Figure 1.35 Ultrasonic pressure fields generated by circular (left top), square (right top) and focused (bottom) transducers. Thin dashed curves in top left and bottom figures have been generated by the closed form expressions [Eq.(1.16b) for the flat circular transducer and Eq.(1.32) for the focused transducer]. Continuous curves are obtained by the DPSM technique.

\section{Solution}

The area for each point source $\left(A_{S}\right)$ is computed from the surface area of the transducer face in the following manner:

$$
A_{\mathrm{S}}=\frac{\pi r^{2}}{n}=\frac{\pi 1.27^{2}}{259}=0.01956 \mathrm{~mm}^{2}
$$

Because $A_{\mathrm{S}}=2 \pi r_{\mathrm{S}}^{2}$ (see Fig. 1.24 and also the discussion on Eqs. (1.29) and (1.30)), we can write,

$$
r_{\mathrm{S}}=\sqrt{\frac{0.01956}{2 \pi}}=0.0558 \mathrm{~mm}
$$

From the wavelength $\left(\lambda_{\mathrm{f}}\right)$, wave speed $\left(c_{\mathrm{f}}\right)$, and frequency $(f)$ relation, we get the wavelength in water for $1 \mathrm{MHz}$ frequency

$$
\lambda_{\mathrm{f}}=\frac{c_{\mathrm{f}}}{f}=\frac{1.5 \times 10^{6}}{10^{6}}=1.5 \mathrm{~mm}
$$




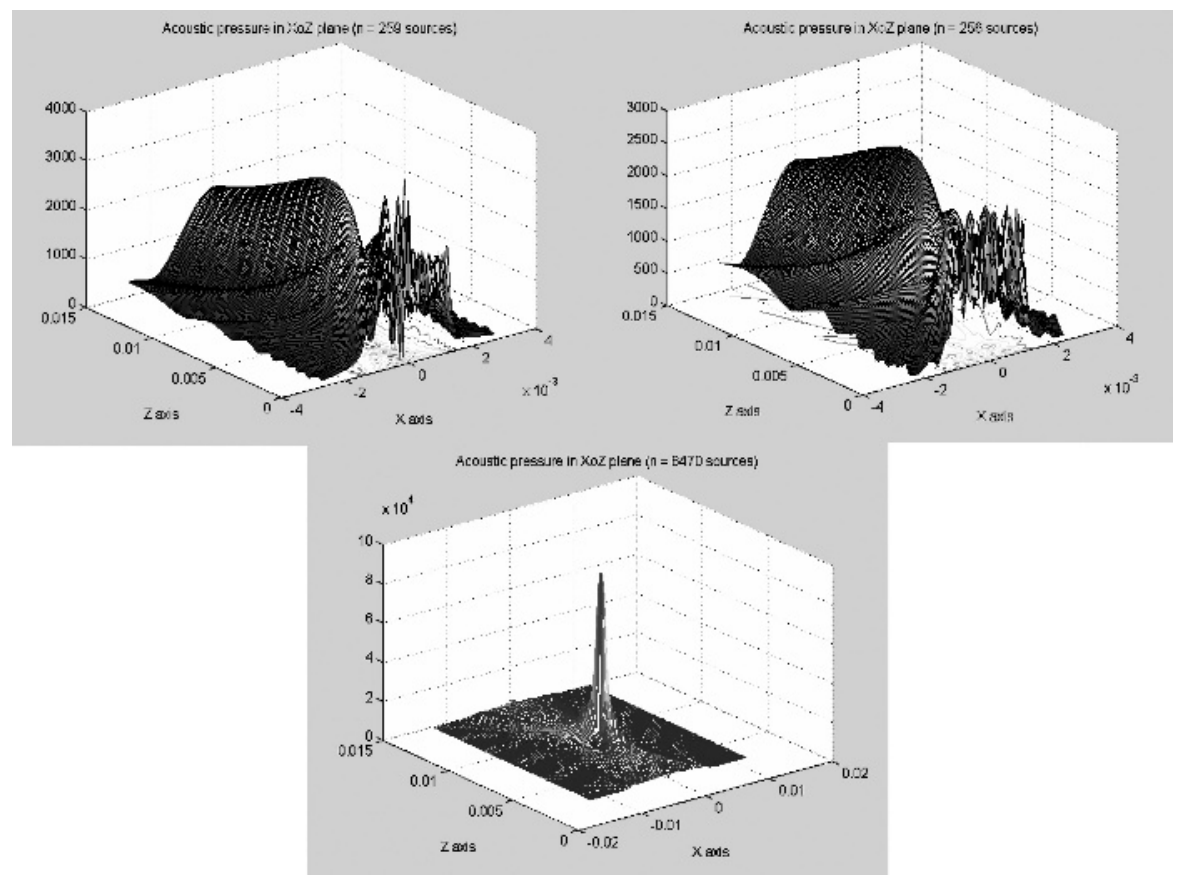

Figure 1.36 Ultrasonic pressure fields in the $x z$ plane are generated by circular (top left), square (top right) and focused (bottom) transducers. The central axis of the transducer coincides with the z-axis.

From Eq. (1.30),

$$
\begin{aligned}
r_{\mathrm{S}} & \ll \frac{1.5}{2 \pi} \mathrm{mm} \\
& \Rightarrow r_{\mathrm{S}} \ll 0.24 \mathrm{~mm}
\end{aligned}
$$

Because $r_{\mathrm{S}}=0.0558 \mathrm{~mm}$, the above condition is satisfied.

Pressure field variations in front of the transducer face along the $x z$ plane for the three transducer geometries of Figure 1.33 are shown in Figure 1.36. In this figure one can clearly see how the pressure field oscillates near the transducer face and decays laterally (in the positive and negative $x$ directions) and axially (in the $z$ direction) for the flat transducers. For the focused transducer a clear peak can be observed near the focal point. Contour plots for the pressure field variations in the $x z$ plane for the same three transducers are shown in Figure 1.37.

Figure 1.38 shows the effect of increasing the number of point sources. As more sources are considered, the computed field becomes smoother. Because the oscillating velocity amplitudes at the transducer surface are different for the left and right columns of Figure 1.38 and so are the scales along the vertical axes, the numerical values in the two columns should not be compared. However, a comparison of the 


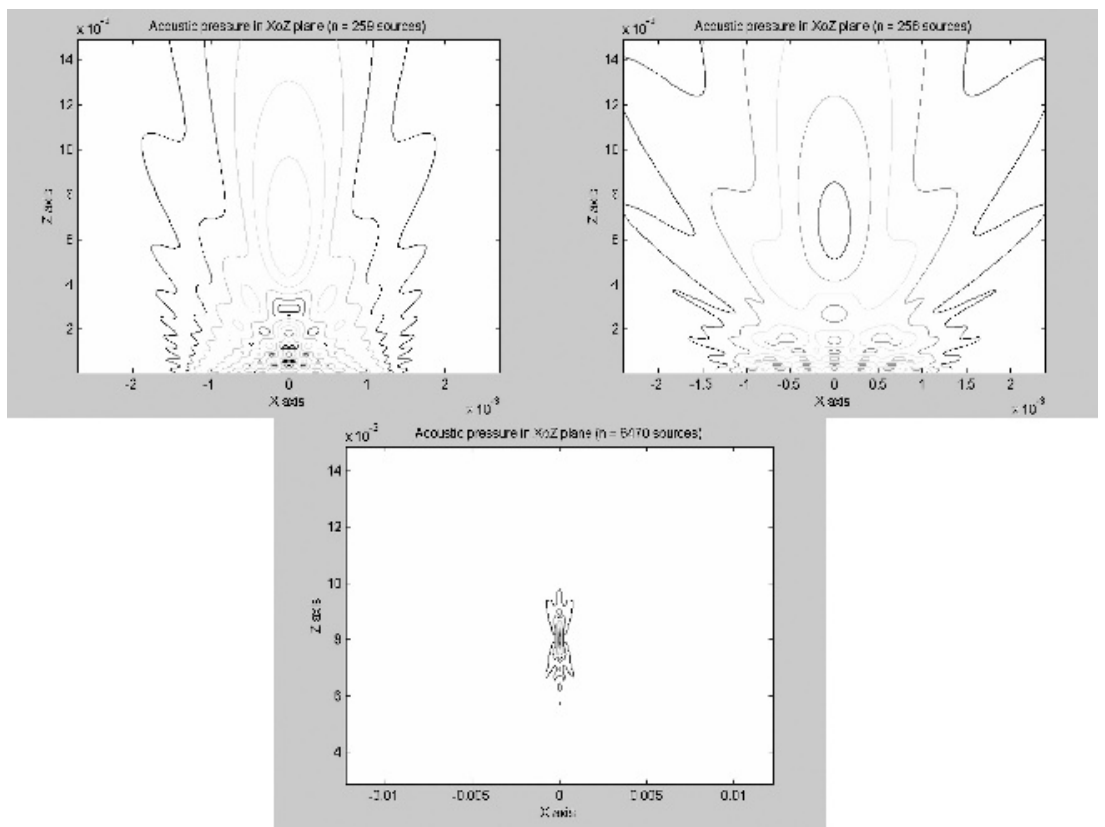

Figure 1.37 Contour plots for the ultrasonic pressure fields in the $x z$ plane are generated by circular (top left), square (top right) and focused (bottom) transducers. The same as Fig. 1.36 but contour plots are given here instead of surface plots.

relative variations of the pressure fields between the two columns clearly demonstrates the effect of the increasing number of point sources on the computed pressure field.

The effect of the presence of a small circular hole at the center of a $2.54 \mathrm{~mm}$ (0.1 in.) diameter flat circular transducer is shown in Figure 1.39. The pressure field in the $x z$ plane (top-right plot of Fig. 1.39) is very similar to the one given in Figure 1.37 (top left). Therefore, a small hole at the center of a flat circular transducer does not significantly affect the generated pressure field in the fluid. The bottom two plots of Figure 1.39 show the pressure and normal velocity $\left(V_{z}\right)$ variations in the $x y$ plane, very close to the transducer surface. It should be noted here that an oscillating pattern is present in the pressure plot but not in the velocity plot. Theoretically, the velocity component should be a constant and equal to $v_{0}$ on the transducer surface, see Eq. (1.16) and (1.25c). However, a small level of noise in the velocity plot exists due to the numerical error.

1.3.7.2 Ultrasonic field in a nonhomogeneous fluid - DPSM technique The pressure field generated by a circular transducer placed parallel to the interface of two fluids is computed. As before, the transducer frequency is set at $1 \mathrm{MHz}$ and its diameter is $2.54 \mathrm{~mm}$. The distance between the transducer face and the interface between two fluids is $10 \mathrm{~mm}$. The transducer is immersed in fluid 1 ( $\mathrm{P}$-wave 


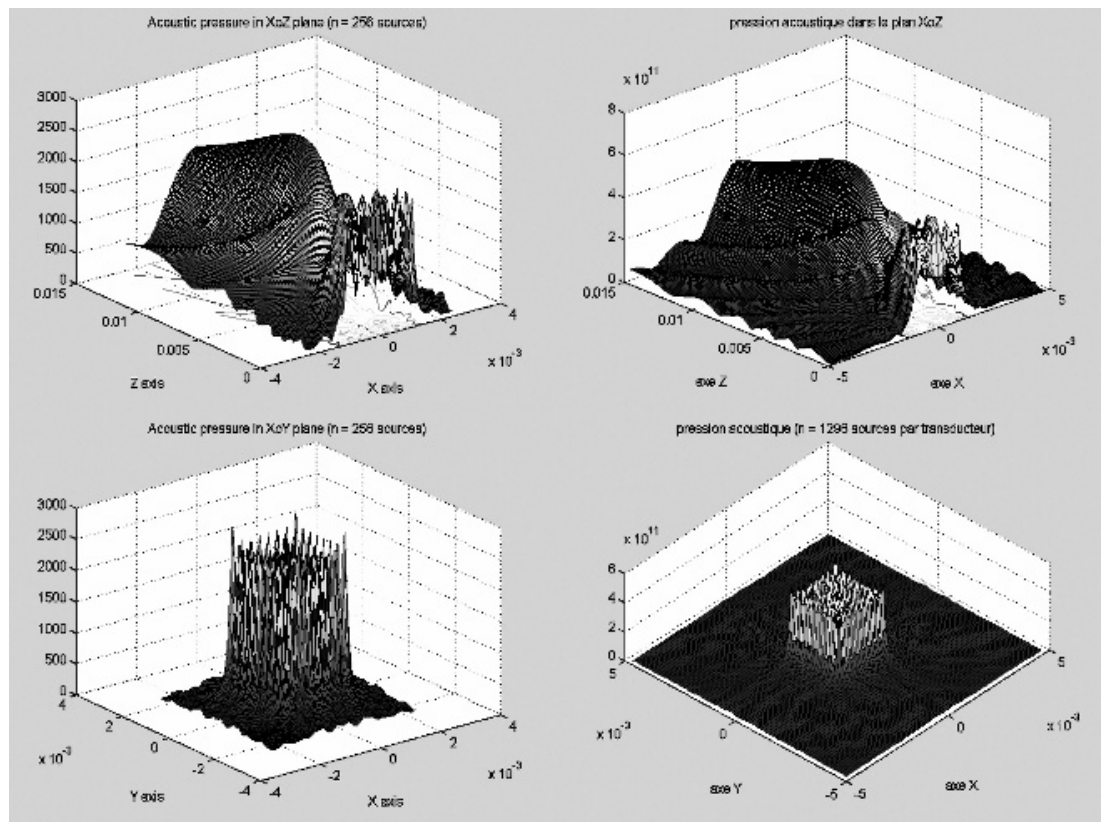

Figure 1.38 Pressure variations in the $x z$ plane (top row) and $x y$ plane (bottom row), close to the transducer surface for a rectangular transducer. Left (256 point sources) and right (1296 point sources) columns correspond to two different discretizations of the transducer surface.

speed $=1.49 \mathrm{~km} / \mathrm{s}$, density $=1 \mathrm{~g} / \mathrm{cc}$ ). The P-wave speed and density of fluid 2 are set at $2 \mathrm{~km} / \mathrm{s}$ and $1.5 \mathrm{~g} / \mathrm{cc}$, respectively.

One hundred point sources are used to model the transducer surface and four hundred point sources (each point source is a triplet source) model the interface effect, see Figures 1.28 and 1.29. Point sources distributed over the interface, which are also called target sources, are distributed over a square area of $20 \mathrm{~mm}$ side length. Note that the interface source positions change (see Figs. 1.29 and 1.30) when computing the acoustic fields in fluids 1 and 2.

Pressure fields computed in the two fluids are plotted in Figure 1.40. Note how the pressure variation in the $x y$ plane is changed, as the distance of the observation ( $x y)$ plane from the transducer surface is increased from zero (middle-left figure) to $10 \mathrm{~mm}$ (bottom-left figure). Pressure variations in the $x z$ plane in both fluids are shown as a contour plot (top-right) and a surface plot (middle-right). Pressure along the $z$-axis is plotted in the bottom-right figure. Oscillations in the acoustic pressure in fluid 1 are the effects of constructive and destructive interferences between two rays that can reach a point in fluid 1 - the first ray travels from the transducer face to the point of interest and the second ray reaches the same point after being reflected at the interface, see Figure 1.27.

Pressure and velocity variations in the two fluids for an inclined transducer (inclination angle $=20^{\circ}$ ) are shown in Figure 1.41. The fluid properties and the 


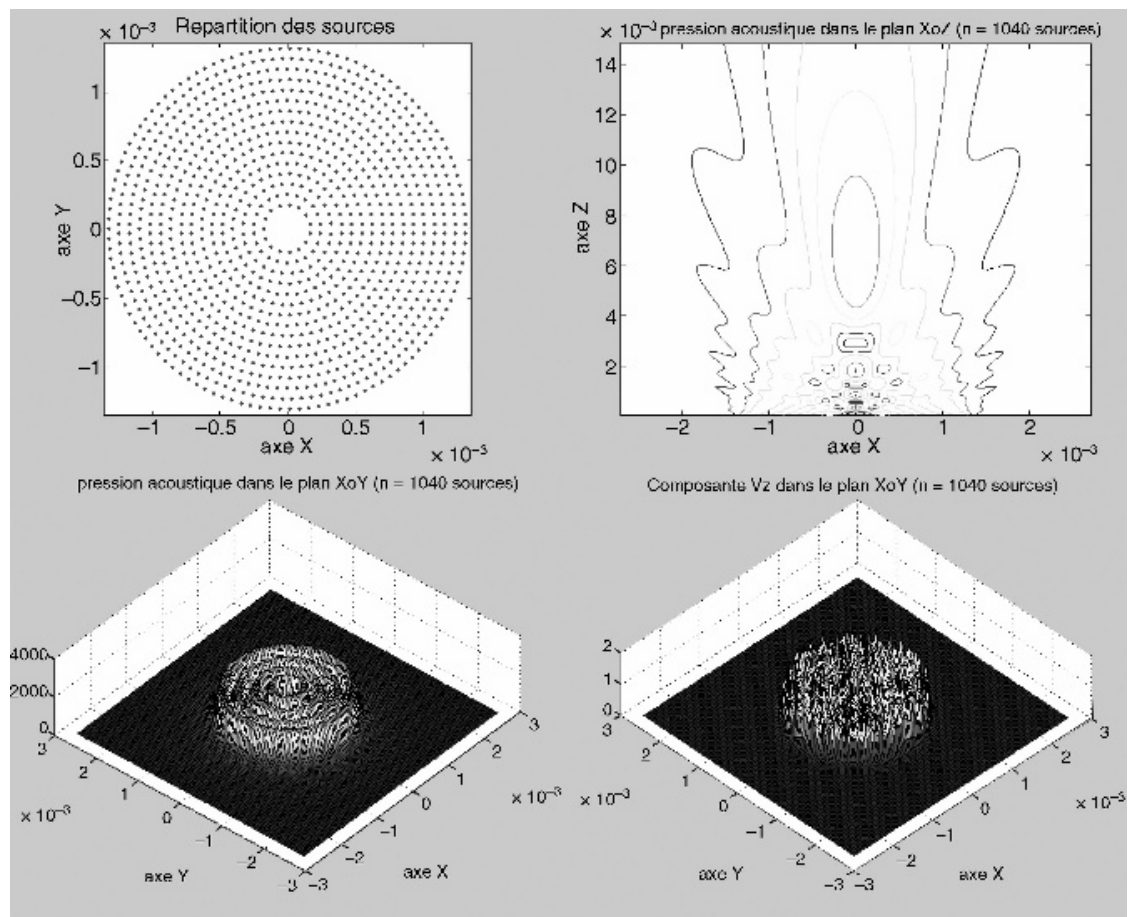

Figure 1.39 Top left - Circular transducer with a small hole at the center is modeled by 1040 point sources; top right - pressure field in the $x z$ plane; bottom left - pressure field in the $x y$ plane, close to the transducer surface; bottom right - normal velocity component $\left(V_{z}\right)$ in the $x y$ plane, close to the transducer surface.

transducer dimension are the same as those in Figures 1.40 and 1.41. The only difference between the problem geometries of Figures 1.40 and 1.41 is that in Figure 1.40 the transducer face is parallel to the interface and in Figure 1.41 it is inclined.

From Snell's law the transmission angle in the second fluid can be computed.

$$
\theta_{\mathrm{T}}=\sin ^{-1}\left(\frac{2}{1.49} \sin (20)\right)=27.33^{\circ}
$$

Incident and transmission angles, measured from the middle-left plot of Figure 1.41, give values close to $20^{\circ}$ and $27.33^{\circ}$, respectively. Note that the $V_{z}$ variation (bottomright) and the pressure variation (middle-right) in the two fluids are similar.

1.3.7.3 Ultrasonic field in a nonhomogeneous fluid-surface integralmethod The ultrasonic field in the nonhomogeneous fluid can also be computed by the conventional surface integral technique instead of the DPSM technique. Unlike the DPSM technique, in the surface integral method the fluid-fluid interface is not modeled by the 


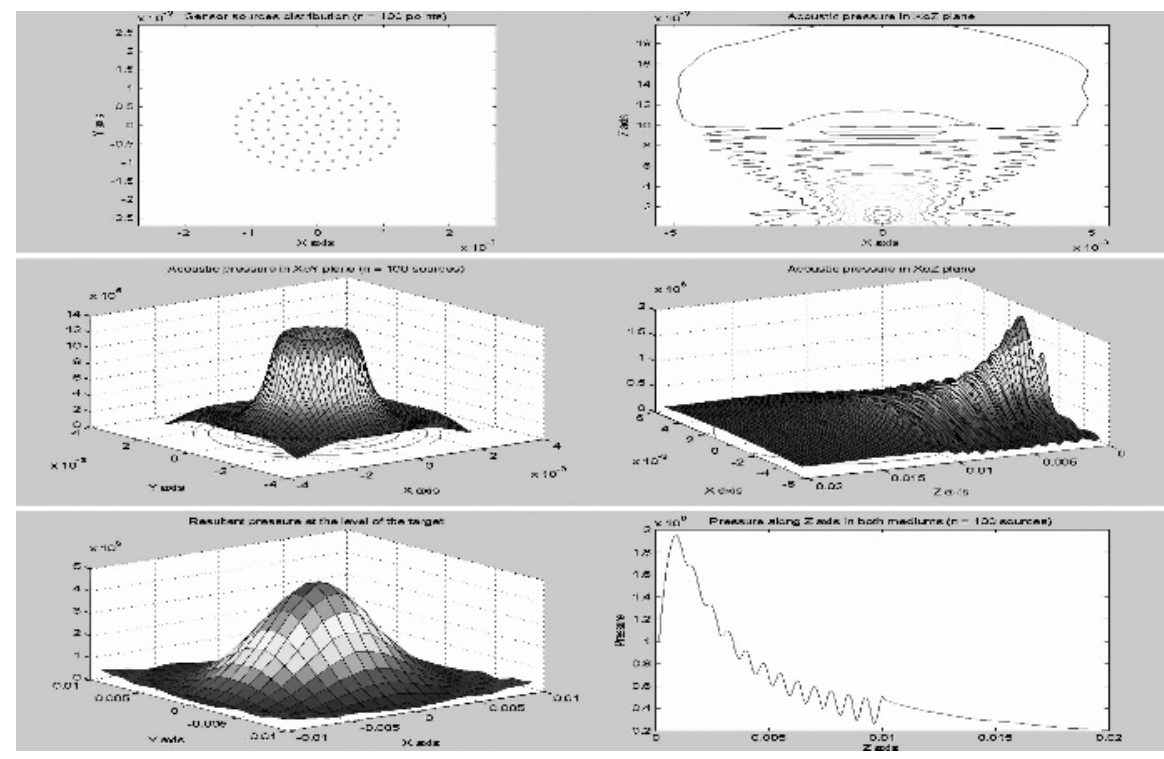

Figure 1.40 Circular transducer in a non-homogeneous fluid. Top left: $2.54 \mathrm{~mm}$ diameter transducer modeled by 100 point sources; middle left - acoustic pressure in the $x y$ plane, close to the transducer surface $(\mathrm{z} \sim 0 \mathrm{~mm})$; bottom left - acoustic pressure in the $x y$ plane in fluid 1 at the interface position $(\mathrm{z}=10 \mathrm{~mm})$; top right - contour plot of the pressure variation in fluid $1(\mathrm{z}=0$ to $10 \mathrm{~mm}$ ) and fluid 2 ( $\mathrm{z}=10$ to $20 \mathrm{~mm}$ ); middle right - surface plot of the pressure variation in fluid $1(\mathrm{z}=0$ to $10 \mathrm{~mm})$ and fluid $2(\mathrm{z}=10$ to $20 \mathrm{~mm})$; bottom right - pressure variation along the $\mathrm{z}$-axis in fluid $1(\mathrm{z}=0$ to $10 \mathrm{~mm})$ and fluid $2(\mathrm{z}=10$ to $20 \mathrm{~mm})$.

distributed point sources. Here, only the transducer surface is discretized into the distributed point sources. In this method, the pressure fields in fluids 1 and 2 are computed by Eqs. (1.45) and (1.60), respectively. The theory of this computation is given in Section 2.4 whereas the theory of the DPSM computation is given in Section 2.5. Figure 1.42 shows the pressure field along the $z$-axis in fluids 1 and 2 , computed by the surface integral technique. A comparison of Figure 1.42 with the bottom-right plot of Figure 1.40 shows a perfect matching between the results obtained by these two methods.

1.3.7.4 Ultrasonic field in the presence of a finite-size scatterer Following the theory described in Section 1.3.6, a computer code has been developed to compute the ultrasonic pressure field in the presence of a finite-size scatterer. This computer code is used to solve the problem of ultrasonic field scattering by a finite-size steel plate, immersed in water. The problem geometry is shown in Figure 1.43. A finite-size thin steel plate ( $1 \mathrm{~mm}$ thick) is placed at the interface between the two fluids-fluid 1 and fluid 2 . The results are presented for the case in which both fluids are water. Scattered fields are computed for a large plate $(20 \mathrm{~mm} \times 20 \mathrm{~mm}$, shown by the dashed line in Fig 1.43), and for a small plate $(5 \mathrm{~mm} \times 5 \mathrm{~mm}$ shown by the solid line in Fig 1.43). 


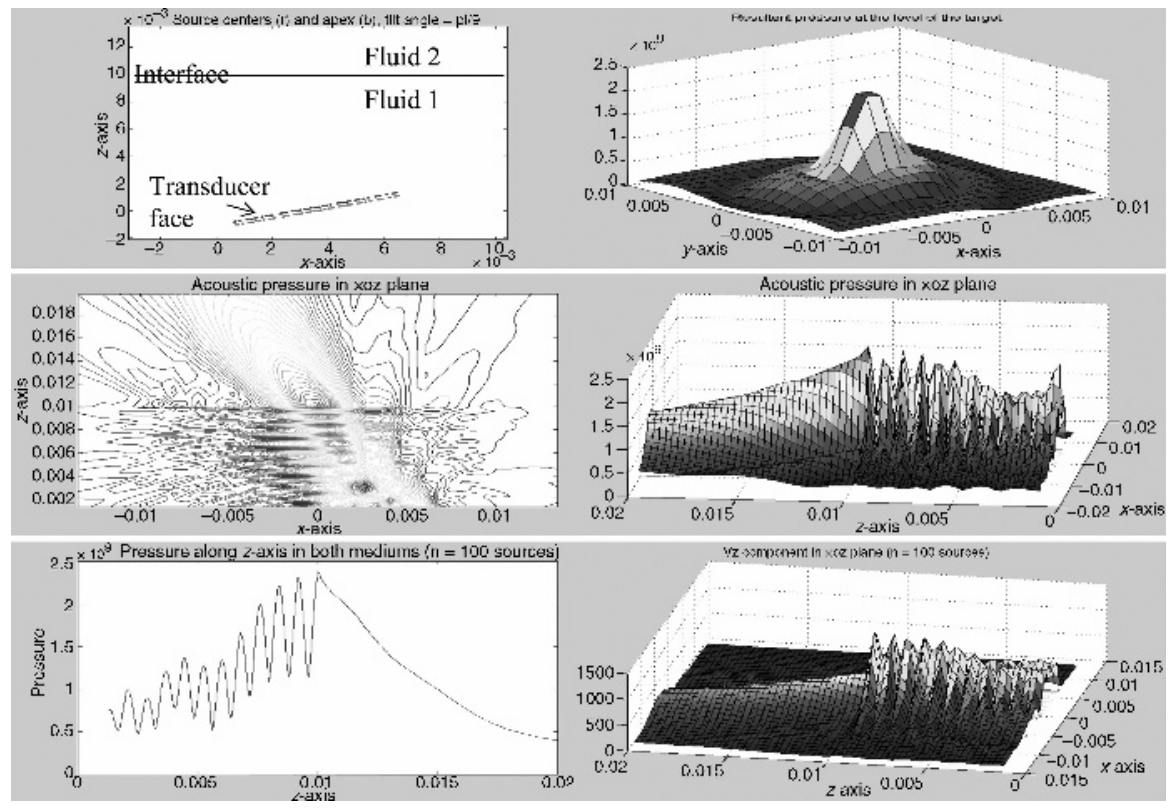

Figure 1.41 Top left: Inclined transducer face modeled with 100 point sources, angle between the interface and the transducer face is $20^{\circ}$; top right - acoustic pressure in the $x y$ plane in fluid 1 at the interface position $(\mathrm{z}=10 \mathrm{~mm})$; middle left - contour plot of the pressure field variation in fluid $1(\mathrm{z}=1$ to $10 \mathrm{~mm})$ and fluid $2(\mathrm{z}=10$ to $20 \mathrm{~mm})$; middle right - surface plot of the pressure field variation in fluid $1(\mathrm{z}=1$ to $10 \mathrm{~mm})$ and fluid $2(\mathrm{z}=10$ to $20 \mathrm{~mm})$; bottom left - pressure variation along the $\mathrm{z}$-axis in fluid $1(\mathrm{z}=1$ to $10 \mathrm{~mm})$ and fluid $2(\mathrm{z}=10$ to $20 \mathrm{~mm})$; bottom right surface plot of the velocity $\left(V_{z}\right)$ variation in fluid $1(\mathrm{z}=1$ to $10 \mathrm{~mm})$ and fluid $2(\mathrm{z}=10$ to $20 \mathrm{~mm})$.

The ultrasonic beam, generated by a $6.28 \mathrm{~mm}$ diameter cylindrical transducer, strikes the plate at angles $\theta_{i}=25^{\circ}$ and $38.37^{\circ}$. Signal frequency is $1 \mathrm{MHz}$. The ultrasonic fields for these two striking angles are computed and plotted in Figures 1.44 and 1.45, respectively (Placko et al., 2003). Material properties for this computation are shown in Table 1.2.

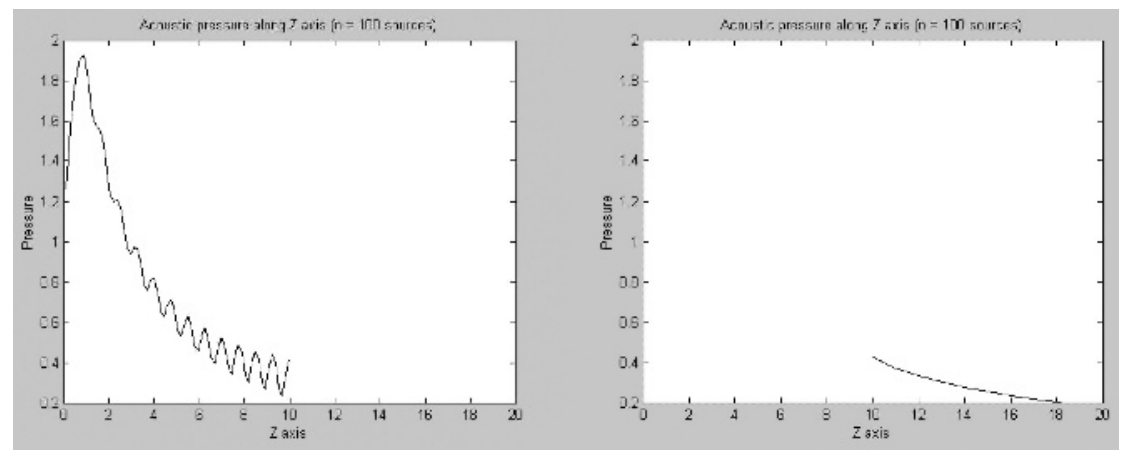

Figure 1.42 Pressure variation along the $\mathrm{z}$-axis in fluid 1 (left figure) and fluid 2 (right figure). 


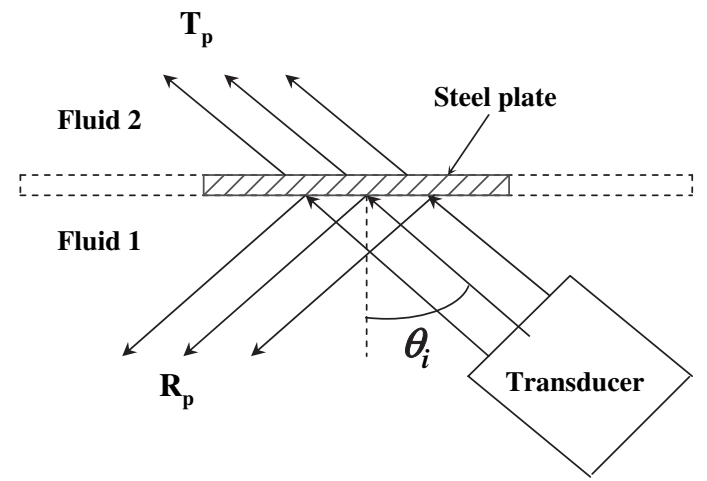

Figure 1.43 A bounded ultrasonic beam from an inclined transducer strikes a finite steel plate immersed in water at an angle $\theta_{i}$ (numerical results are provided for fluid $1=$ fluid $2=$ water).

The plate is placed at a distance of $10 \mathrm{~mm}$ from the transducer face. Thirty-two point sources distributed slightly behind the transducer face, as shown in Figure 1.24, model the transducer.

Note that in both Figures 1.44 and 1.45, scattered fields behind the steel plate are much stronger for the small plate. For the large steel plate, very little acoustic energy

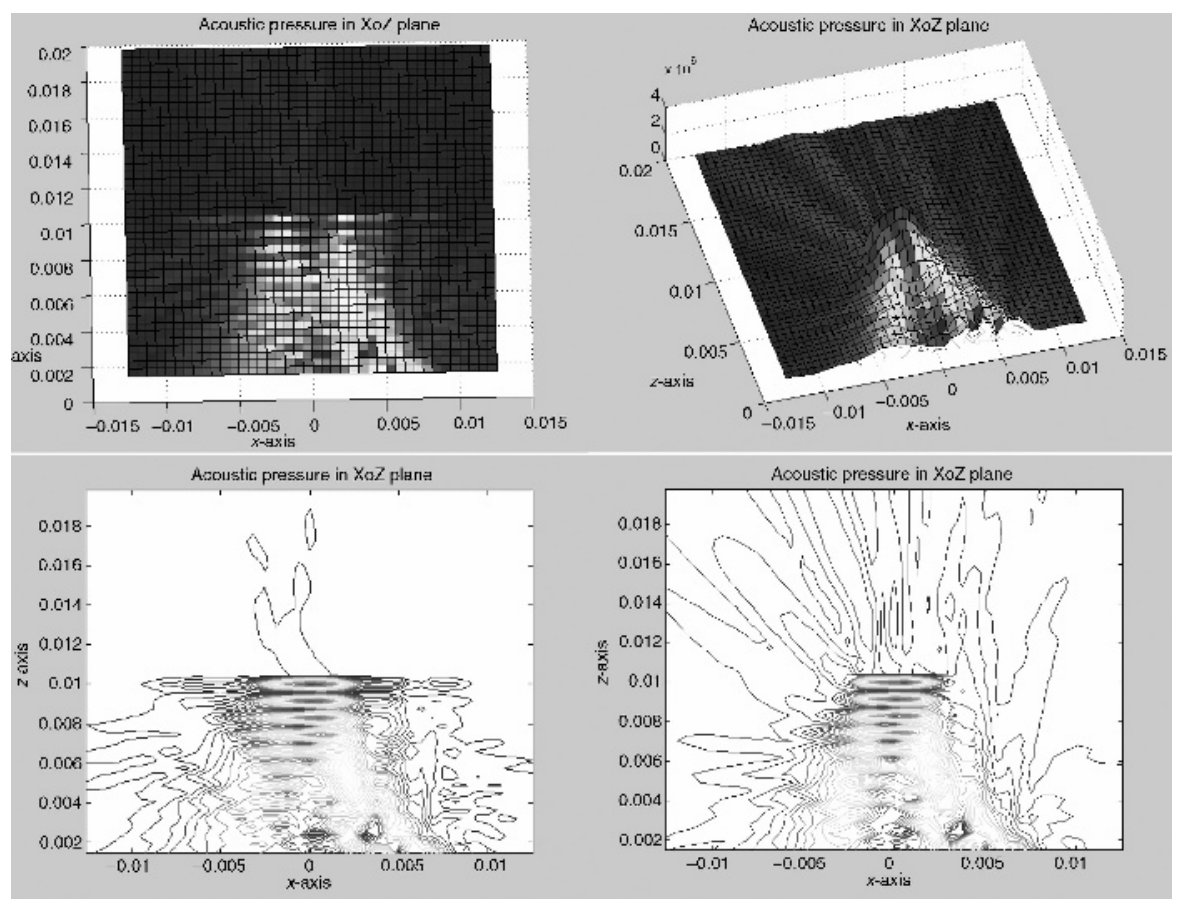

Figure 1.44 Total ultrasonic pressure distributions (incident plus scattered fields) near a steel plate scatterer, immersed in water. Left and right columns are for large $(20 \mathrm{~mm} \times 20 \mathrm{~mm})$ and small $(5 \mathrm{~mm} \times 5 \mathrm{~mm})$ plates, respectively. Incident angle is $25^{\circ}$. In top and bottom rows, the same pressure fields are plotted in two different ways. 


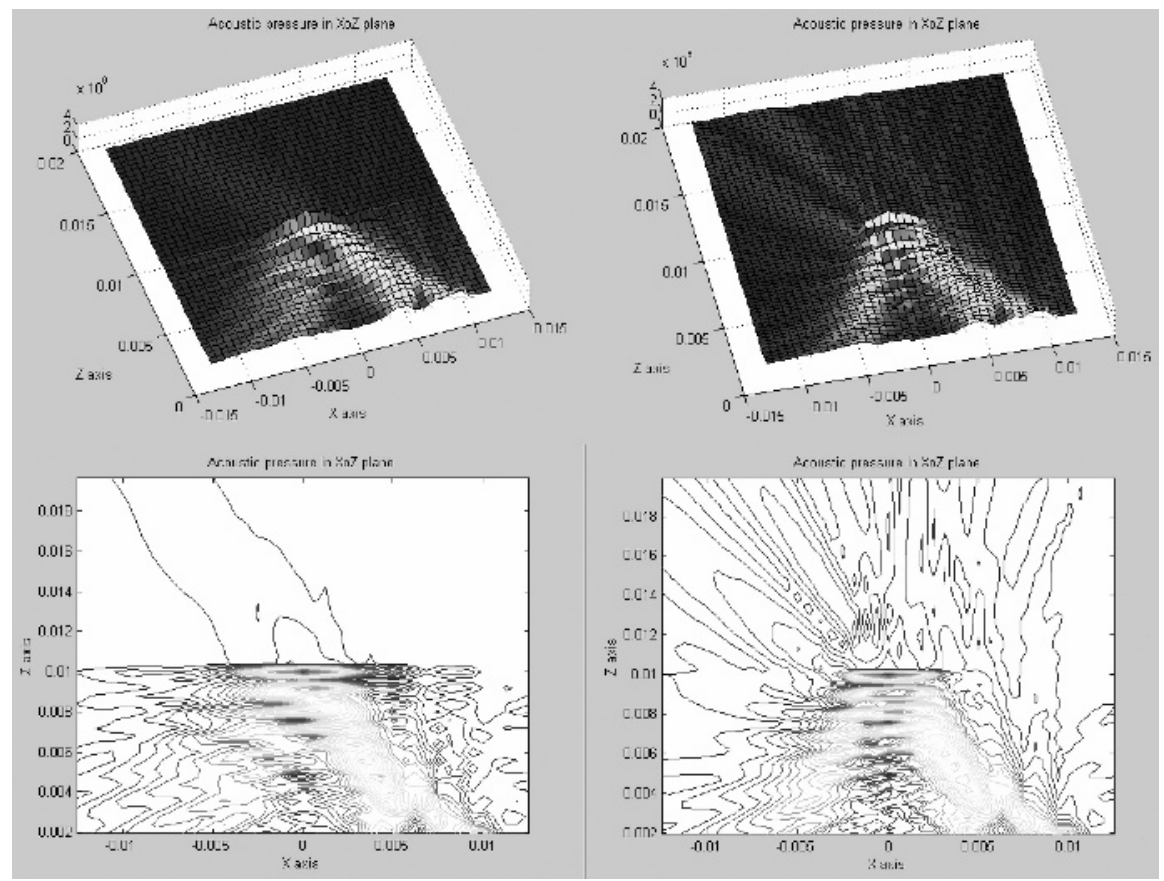

Figure 1.45 Same as Fig.1.44, but these plots are for $38.37^{\circ}$ angle of incidence

is transmitted into the fluid, behind the plate, because of the large impedance mismatch between the steel plate and the water. It should also be noted that in addition to the transmitted field, the reflected field for the large plate is also relatively weak. The weak specular reflection for the large plate is more evident in Figure 1.45. Specular reflected beam means the reflected beam in the position predicted by the optics theory. The probable cause for a weak specular reflection by the large plate is that part of the ultrasonic energy generates leaky guided waves in the plate and propagates away from the striking zone. Therefore, less energy is specularly reflected by the larger plate. In Figure 1.46 we can see that for $38.37^{\circ}$ incident angle, a guided wave mode is generated; thus, less energy is specularly reflected for this incident angle when the plate is large. This phenomenon of guided wave generation at the fluid-solid interface is discussed in detail in chapter 4 .

TABLE 1.2. Water and steel properties for the results presented in Figures 1.44 and 1.45

\begin{tabular}{lccc}
\hline $\begin{array}{l}\text { Material and } \\
\text { Properties }\end{array}$ & $\begin{array}{c}\text { P-wave } \\
\text { Speed }(\mathrm{km} / \mathrm{s})\end{array}$ & $\begin{array}{c}\text { S-wave } \\
\text { Speed }(\mathrm{km} / \mathrm{s})\end{array}$ & Density $(\mathrm{g} / \mathrm{cc})$ \\
\hline Steel & 5.96 & 3.26 & 7.93 \\
Water & 1.49 & - & 1 \\
\hline
\end{tabular}




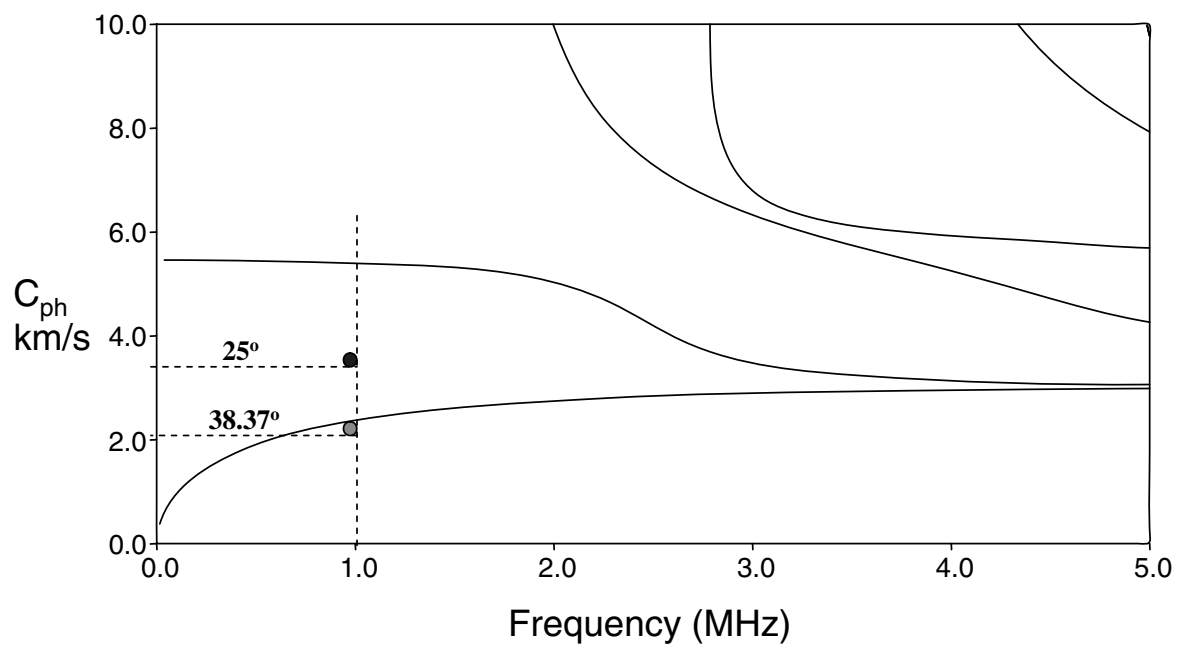

Figure 1.46 Dispersion curves for $1 \mathrm{~mm}$ thick steel plate (properties given in Table 1.2). Phase velocities corresponding to the two striking angles of Figs.1.44 $\left(25^{\circ}\right)$ and $1.45\left(38.37^{\circ}\right)$ are shown in the figure. Note that $38.37^{\circ}$ incidence is capable of generating guided wave in the plate.

\section{REFERENCES}

ANSYS Inc., “ANSYS 5.6 Online Help", copyright 1999.

Ahmad, R., T., Kundu, and D., Placko, "Modeling of the Ultrasonic Field of Two Transducers Immersed in a Homogeneous Fluid Using Distributed Point Source Method", I2M (Instrumentation, Measurement and Metrology) Journal, Vol. 3, pp. 87-116, 2003.

Ahmad, R., T., Kundu, and D., Placko, "Modeling of Phased Array Transducers", Journal of the Acoustical Society of America, Vol. 117, pp. 1762-1776, 2005.

Banerjee, S., T., Kundu, and D., Placko, "Ultrasonic Field Modelling in Multilayered Fluid Structures Using DPSM Technique", ASME Journal of Applied Mechanics, Vol. 73 (4), pp. 598-609, 2006.

Dufour, I., and D., Placko, “An Original Approach of Eddy Current Problems Through a Complex Electrical Image Concept”, IEEE Transactions on Magnetics, Vol. 32 (2), pp. 348 365, 1996.

Kundu, T., Chapter 1: Mechanics of Elastic Waves and Ultrasonic NDE, in Ultrasonic Nondestructive Evaluation: Engineering and Biological Material Characterization, Ed. T. Kundu, Pub. CRC Press, 2004.

Lee, J. P., Placko, D., Alnuamimi, N., and Kundu, T., "Distributed Point Source Method (DPSM) for Modeling Ultrasonic Fields in Homogeneous and Non-Homogeneous Fluid Media in Presence of an Interface", First European Workshop on Structural Health Monitoring, Ed. D. Balageas, Ecole Normale Superieure de Cachan, France, July 10-12, 2002.

Lemistre, M., and Placko, D., "Procédé et dispositif électromagnétique pour le contrôle de structures intégré", Patent Application $\mathrm{N}^{\circ}$ BFF 040003 ENS Cachan/CNRS/ONERA, March 31, 2004, European extension March 31, 2005. 
Placko, D., "Dispositifs d'analyse de profil utilisant des capteurs à courants de Foucault", Thèse de 3ème cycle, University Paris XI, April 16, 1984 (in French).

O'Neil, H.T., "Theory of Focusing Radiators", Journal of the Acoustical Society of America, Vol. 21 (5), pp. 516-526, 1949.

Placko, D., Clergeot, H., and Monteil, F., "Seam tracking using a linear array of eddy current sensors", Proceedings ROVISEC 5, pp. 557-568, Amsterdam, October 1985.

Placko, D., Clergeot, H., and Santander, E., "Physical Modeling of an eddy current sensor designed for real time distance and thickness measurement in galvanisation industry", IEEE Transactions on Magnetics, Vol. 25 (4), pp. 2861-2863, 1989.

Placko, D., "Contribution à la conception de capteurs inductifs pour la robotique industrielle", Diplôme d'Habilitation à Diriger des Recherches en Sciences, University Paris XI, May 22, 1990 (in French).

Placko, D., and T., Kundu, "A Theoretical Study of Magnetic and Ultrasonic Sensors: Dependence of Magnetic Potential and Acoustic Pressure on the Sensor Geometry", Advanced NDE for Structural and Biological Health Monitoring, Proceedings of SPIE, Ed. T. Kundu, SPIE's 6th Annual International Symposium on NDE for Health Monitoring and Diagnostics, March 4-8, 2001, Newport Beach, California, Vol. 4335, pp. 52-62, 2001.

Placko, D., Kundu, T., and Ahmad, R., "Distributed Point Source Method (DPSM) for Modeling Ultrasonic Fields in Homogeneous and Non-Homogeneous Fluid Media", Second International Conference on Theoretical, Applied, Computational and Experimental Mechanics, Kharagpur, India, December 27-30, 2001, Pub. Globenet Computers, India, Published in CD, Paper No. 114, 2001.

Placko, D., Liebeaux, N., and Kundu, T., "Presentation d'une methode generique pour La Modelisation des Capteurs de type Ultrasons, Magnetiques et Electrostatiques", Instrumentation, Mesure, Metrologie - Evaluation nondestructive, Vol. 1, pp. 101-125, 2001 (in French).

Placko, D., Kundu, T., and Ahmad, R., "Theoretical Computation of Acoustic Pressure Generated by Ultrasonic Sensors in Presence of an Interface", Smart NDE and Health Monitoring of Structural and Biological Systems, Ed. T. Kundu, SPIE's 7th Annual International Symposium on NDE for Health Monitoring and Diagnostics, March 18-21, 2002, San Diego, California, pp. 157-168, Vol. 4702, 2002.

Placko D., Liebeaux N., Kundu T., "Procédé pour évaluer une grandeur physique representative d'une interaction entre une onde et un obstacle", Patent Application $N^{\circ} 0214108$ ENS Cachan/CNRS/Université d'Arizona, November 8, 2002. European and USA Extension November 10, 2003.

Placko, D., and Kundu, T., Chapter 2: Modeling of Ultrasonic Field by Distributed Point Source Method, in Ultrasonic Nondestructive Evaluation: Engineering and Biological Material Characterization, Ed. T. Kundu, Pub. CRC Press, pp. 143-202, 2004.

Placko, D., T. Kundu, and R. Ahmad, "Ultrasonic Field Computation in Presence of a Scatterer of Finite Dimension", Smart Nondestructive Evaluation and Health Monitoring of Structural and Biological Systems, Ed. T. Kundu, SPIE's 8th Annual International Symposium on NDE for Health Monitoring and Diagnostics, March 3-5, 2003, San Diego, California, Vol. 5047, 2003.

Schmerr L.W., Fundamentals of Ultrasonic Nondestructive Evaluation-A Modeling Approach, Pub. Plenum Press, 1998. 